\title{
Discovery of a Third Transiting Planet in the Kepler-47 Circumbinary System
}

\author{
Jerome A. Orosz ${ }^{1}$ (1), William F. Welsh ${ }^{1}$ (1) , Nader Haghighipour ${ }^{2}$ (1), Billy Quarles ${ }^{3}$, Donald R. Short ${ }^{1}$, Sean M. Mills ${ }^{4,5}$ (1),
} Suman Satyal ${ }^{6}$, Guillermo Torres ${ }^{7}$ (10, Eric Agol $^{8}$ (1), Daniel C. Fabrycky ${ }^{4}$ (i), Daniel Jontof-Hutter, ${ }^{9}$ (10), Gur Windmiller ${ }^{1}$,

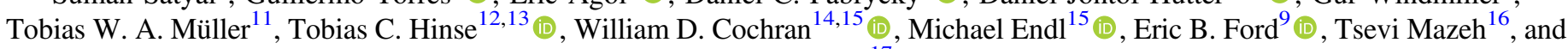
Jack J. Lissauer ${ }^{17}$

\footnotetext{
${ }^{1}$ Astronomy Department, San Diego State University, 5500 Campanile Drive, San Diego, CA 92182-1221, USA; jorosz@sdsu.edu

${ }^{2}$ Institute for Astronomy, University of Hawaii-Manoa, 2680 Woodlawn Dr., Honolulu, HI 96822, USA

${ }^{3}$ Homer L. Dodge Department of Physics \& Astronomy, University of Oklahoma, 440 W. Brooks Street, Norman, OK 73019, USA

${ }^{4}$ The Department of Astronomy and Astrophysics, The University of Chicago, 5640 S. Ellis Ave., Chicago, IL 60637, USA

${ }^{5}$ Department of Astronomy, California Institute of Technology, 1200 E. California Blvd., Pasadena, CA 91125, USA

${ }^{6}$ The Department of Physics, University of Texas at Arlington, 502 Yates Street, Arlington, TX 76019, USA

${ }^{7}$ Harvard-Smithsonian Center for Astrophysics, 60 Garden Street, Cambridge, MA 02138, USA

${ }^{8}$ Department of Astronomy, University of Washington, Box 351580, 3910 15th Ave NE, Seattle, WA 98195, USA

${ }^{9}$ Department of Astronomy \& Astrophysics, The Pennsylvania State University, 525 Davey Lab, University Park, PA 16082, USA

${ }^{10}$ Department of Physics, University of the Pacific, 3601 Pacific Avenue, Stockton, CA 95211, USA

${ }^{11}$ Institute for Astronomy and Astrophysics, University of Tübingen, Auf der Morgenstelle 10, D-72076 Tuebingen, Germany

${ }_{12}$ Department of Astronomy and Space Science, Chungnam National University, Daejeon 34134, Republic of Korea

${ }^{13}$ Korea Astronomy \& Space Science Institute, 776 Daedukdae-ro, Yuseong-gu, 305-348 Daejeon, Republic of Korea

${ }^{14}$ The University of Texas at Austin, Department of Astronomy, 2515 Speedway, Stop C1400, Austin, TX 78712-1205, USA

${ }^{15}$ McDonald Observatory, The University of Texas at Austin, Austin, TX 78712-0259, USA

${ }^{16}$ School of Physics and Astronomy, Tel Aviv University, Tel Aviv 69978, Israel

${ }^{17}$ Space Science and Astrobiology Division, MS 245-3, NASA Ames Research Center, Moffett Field, CA 94035, USA Received 2018 March 24; revised 2019 March 1; accepted 2019 March 1; published 2019 April 16
}

\begin{abstract}
Of the nine confirmed transiting circumbinary planet systems, only Kepler-47 is known to contain more than one planet. Kepler- $47 \mathrm{~b}$ (the "inner planet") has an orbital period of 49.5 days and a radius of about $3 R_{\oplus}$. Kepler-47 c (the "outer planet") has an orbital period of 303.2 days and a radius of about $4.7 R_{\oplus}$. Here we report the discovery of a third planet, Kepler-47 d (the "middle planet"), which has an orbital period of 187.4 days and a radius of about $7 R_{\oplus}$. The presence of the middle planet allows us to place much better constraints on the masses of all three planets, where the $1 \sigma$ ranges are less than $26 M_{\oplus}$, between 7-43 $M_{\oplus}$, and between 2-5 $M_{\oplus}$ for the inner, middle, and outer planets, respectively. The middle and outer planets have low bulk densities, with $\rho_{\text {middle }}<0.68 \mathrm{~g} \mathrm{~cm}^{-3}$ and $\rho_{\text {outer }}<0.26 \mathrm{~g} \mathrm{~cm}^{-3}$ at the $1 \sigma$ level. The two outer planets are "tightly packed," assuming the nominal masses, meaning no other planet could stably orbit between them. All of the orbits have low eccentricities and are nearly coplanar, disfavoring violent scattering scenarios and suggesting gentle migration in the protoplanetary disk.
\end{abstract}

Key words: binaries: eclipsing - planets and satellites: detection - planets and satellites: fundamental parameters stars: fundamental parameters

Supporting material: machine-readable tables

\section{Introduction}

Binary stars are common: for example, roughly half of all Sun-like stars are found in pairs (Raghavan et al. 2010). Circumbinary planets (hereafter, CBPs), i.e., planets that orbit an entire binary star system, can reveal their presence in a variety of different ways. Known as P-type systems (Dvorak 1982), these planets, if sufficiently large, may affect radial velocities of the stars or the timing of stellar eclipses (Schwarz et al. 2011). Several claims of the detection of CBPs using eclipse timing variations have been made (for example Qian et al. 2012a, 2012b, 2013; Lee et al. 2014), but the validity of these detections is currently under question (e.g., Marsh 2018). A CBP may also transit one or both stars in the binary. When the system is viewed edge-on (i.e., as in an eclipsing binary), transits in the light curve provide the most secure detection of the planet. To date, ten transiting CBPs have been discovered with this method (Doyle et al. 2011; Orosz et al. 2012a, 2012b; Welsh et al. 2012, 2015; Kostov et al. 2013, 2014, 2016; Schwamb et al. 2013) in the set of $\sim 2900$ eclipsing binary stars $^{18}$ (Prša et al. 2011; Slawson et al. 2011; Kirk et al. 2016) observed with the Kepler telescope (Borucki et al. 2010a, 2010b; Koch et al. 2010). Transits are particularly valuable because they allow the planet's radius to be measured, and the large variations in transit durations and the deviations from periodicity unambiguously demonstrate that the body is in a circumbinary orbit (Orosz et al. 2012b).

Kepler-47, the topic of this paper, is the only known multiplanet circumbinary system, and the multi-planet nature of this system allows us to study an ensemble of star-planet and planet-planet dynamics. Its binary stars have an orbital period of $\sim 7.5$ days, the shortest known in any CBP system. The innermost planet (Kepler-47 b) has an orbital period of $\sim 49.5$ days, and is the smallest of the known CBPs $\left(\sim 3 R_{\oplus}\right)$. The Uranus-size outer planet (Kepler-47 c) has a $\sim 303$ day orbit, placing it well within the habitable zone (Haghighipour \& Kaltenegger 2013), i.e., the region surrounding a star where water could exist in a liquid state

${ }^{18}$ http://keplerebs.villanova.edu 
on a terrestrial planet (Kopparapu et al. 2013). The existence of the Kepler-47 system, particularly with the newly discovered middle planet described herein, shows that multi-planetary systems can form and survive around close binary stars. This is despite theory suggesting that strong perturbations from the binary on the protoplanetary disk most likely inhibit in situ formation in regions close to the binary (Meschiari 2012; Paardekooper et al. 2012; Dunhill \& Alexander 2013; Marzari et al. 2013; Pierens \& Nelson 2013; Rafikov 2013; Lines et al. 2015a, 2015b, 2016).

In the system discovery paper (Orosz et al. 2012b), eighteen transits of the inner planet and three of the outer planet were detected. An additional $0.2 \%$ deep "orphan" transit event was noted, a feature that could not be attributed to the two planets known at that time. Further Kepler observations revealed two more transits not attributable to the two known planets, suggesting the existence of a third planetary body and allowing for a robust determination of its orbital period. This, in turn, allowed us to identify three much weaker transits in the earlier data. In total, six transits were observed that can be attributed to an additional planet, Kepler-47 d, orbiting between planets $b$ and c. In this work, we provide an updated analysis of the fivebody dynamics. The paper is organized as follows. We discuss the available observational material in Section 2. The transits of the new planet and transit times for all of the planets are discussed in Section 3. Measurements of times when the primary and secondary stars eclipse one another are given in Section 4. Our five-body photodynamical model is described in Section 5. The mass estimates for the planets, the long-term dynamical stability of the system, the evolutionary status of the two stars, and the locations of the middle and outer planets to the habitable zone of the binary are discussed in Section 6. We summarize our results in Section 7.

\section{Observational Data}

\subsection{Optical Light Curves}

Kepler-47 (KIC 10020423, KOI 3154, 2MASS J19411149 $+4655136)$ was observed in long-cadence mode $(\approx 30$ minute samples) from Kepler Quarter 1 (BJD 2,454,964.51 or 2009 May 13) through the end of the nominal spacecraft operation in the second month of Quarter 17 (BJD 2,456,424.00 or 2013 May 11). There were a total of 65,428 long-cadence observations during this span (including flagged data), for a duty cycle of $91.6 \%$. Starting in Quarter 14 (BJD 2,456,107.13 or 2012 June 28), Kepler-47 was on the list of short-cadence targets $(\approx 1$ minute samples), where it remained until the cessation of normal spacecraft operations. There were a total of 409,046 short-cadence observations, including flagged data. We downloaded the FITS tables from the Mikulski Archive for Space Telescopes (MAST). These files were processed using the SOC release 9.0 .3 , and they include a $\approx 1$ minute correction to the time stamps that was not available at the time of original work reported in Orosz et al. (2012b).

The Kepler light curves of Kepler-47 required detrending, owing to instrumental trends and modulations due to star spots. We arrived at our final detrended light curves using a two-step process. First, an interactive technique described in Orosz et al. (2012b) and Bass et al. (2012) was used. In this step, the light curve was broken up into segments using data gaps and sudden jumps in the flux as end points. For each segment, data in the eclipses and transits were masked out, and cubic splines were fit to the remaining data. The segments were normalized by the spline fits, and pieced together to produce a detrended light curve. While effective, this technique is not entirely reproducible; it is also difficult to use with the short-cadence data, owing to limitations in our implementation of the method. Thus, an automated and reproducible detrending algorithm was devised as follows: The light curve that was detrended manually was modeled as described in Section 5. Once a good fit was found, the model was used to precisely determine the times of the eclipses and transits and the durations of these events. The times and durations were then used to determine which data points occurred during an eclipse or a transit event, which allowed us to assign those points zero weight during the normalization.

In our original work, only long-cadence Kepler data were available to us. As noted above, Kepler-47 was on the list of shortcadence targets for Kepler Quarters Q14 through Q17. In our view, using short-cadence data (when available) instead of longcadence data is preferable because the ingress and egress phases of eclipses and transits are better-resolved. Thus, a "raw" light curve using long-cadence data from Quarter 1 through Quarter 13 and short-cadence data from Quarter 14 through Quarter 17 was made, and data with quality flags with values greater than 16 were eliminated (in our experience, obviously poor cadences almost always have data quality flags greater than 16). The overall duty cycle of the retained data was $91.6 \%$. Segments centered at the mid-eclipse and transit times with widths of three times the duration of the event in question were extracted from the raw light curve. In a few cases, these segments included both an eclipse event and a transit event; also in a few cases, the segments were truncated owing to gaps in the data. A fifth-order Legendre polynomial was fit to each segment, where data that occurred during an eclipse or a transit event were given zero weight. The normalized segments were assembled to give the final normalized and trimmed light curve. We also produced a normalized light curve consisting entirely of long-cadence observations for the purposes of plotting the relatively weak transits.

\subsection{Radial Velocities}

Owing to the relative faintness of the secondary star (it is about $0.5 \%$ as bright as the primary in the optical), Kepler- 47 is a single-lined binary, so only radial velocity measurements for the primary star are available. We used 11 radial velocity measurements of the primary in the original work (Orosz et al. $2012 b$ ). Four of these came from the HRS spectrograph on the Hobby-Eberly Telescope (HET), six of these came from the Tull Coudé spectrograph on the Harlan J. Smith $2.7 \mathrm{~m}$ telescope (HJST) at McDonald Observatory, and one measurement came from the HIRES spectrograph on the Keck I telescope. Since that time, Kostov et al. (2013) have provided an additional four measurements with the SOPHIE spectrograph on the $1.93 \mathrm{~m}$ telescope at Haute-Provence Observatory. These measurements are summarized in Table 1 and displayed in Figure 1. Small differences were found in the velocity zero-points between the different observatories, so these were fitted for and removed. To arrive at our final radial velocity curve, we subtracted systemic offsets of $4.5680 \mathrm{~km} \mathrm{~s}^{-1}$ for the HJST radial velocities, $4.5999 \mathrm{~km} \mathrm{~s}^{-1}$ for the HET radial velocities, and $4.2685 \mathrm{~km} \mathrm{~s}^{-1}$ for the Kostov et al. (2013) velocities. Given that we have only one measurement from Keck, we cannot determine the systematic offset and we do not 


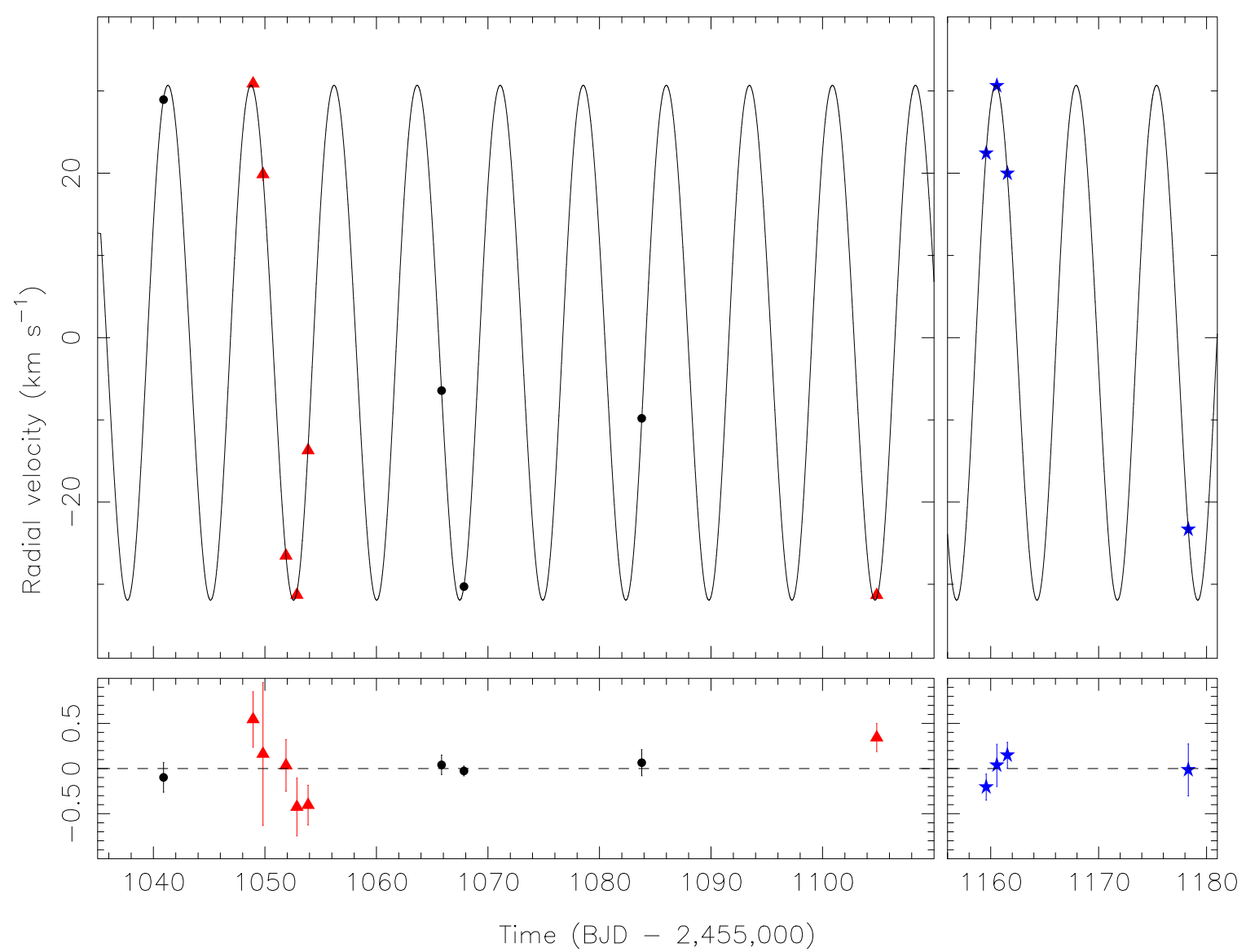

Figure 1. Top: the radial velocity measurements (in $\mathrm{km} \mathrm{s}^{-1}$ ) of the Kepler-47 primary are shown along with the best-fitting model. The horizontal axis gives the time in days, where the abbreviation BJD used here and in subsequent figures means "barycentric Julian date." The black filled circles denote measurements from the Hobby-Eberly Telescope, the red filled triangles denote measurements from the McDonald $2.7 \mathrm{~m}$, and the blue filled stars denote measurements taken from Kostov et al. (2013). For clarity, the model curve in the $\approx 50$ day gap between the radial velocities given in Orosz et al. (2012b) and those given in Kostov et al. (2013) is not shown. Bottom: the residuals of the model fit, where the symbols have the same meaning as in the top panels.

Table 1

Radial Velocities for Kepler-47

\begin{tabular}{|c|c|c|c|c|c|}
\hline $\begin{array}{l}\text { Date } \\
\text { YYYY-MM-DD }\end{array}$ & UT Time & $\begin{array}{c}\text { BJD } \\
(2,455,000+)\end{array}$ & $\begin{array}{c}\mathrm{RV}_{A} \\
\left(\mathrm{~km} \mathrm{~s}^{-1}\right)^{\mathrm{a}}\end{array}$ & $\begin{array}{c}\mathrm{RV}_{A} \\
\left(\mathrm{~km} \mathrm{~s}^{-1}\right)^{\mathrm{b}}\end{array}$ & Telescope \\
\hline 2012 Apr 10 & $13: 25: 48.68$ & 1028.05942 & $11.442 \pm 0.011$ & $\ldots$ & Keck \\
\hline 2012 Apr 23 & 09:11:27.36 & 1040.90325 & $33.534 \pm 0.091$ & $28.934 \pm 0.164$ & HET \\
\hline 2012 May 1 & 09:52:55.08 & 1048.93237 & $35.458 \pm 0.171$ & $30.890 \pm 0.308$ & HJST \\
\hline 2012 May 2 & 07:23:45.95 & 1049.82882 & $24.430 \pm 0.440$ & $19.862 \pm 0.792$ & HJST \\
\hline 2012 May 4 & 08:34:10.40 & 1051.88474 & $-21.957 \pm 0.159$ & $-26.525 \pm 0.286$ & HJST \\
\hline 2012 May 5 & 08:08:26.83 & 1052.86692 & $-26.719 \pm 0.178$ & $-31.287 \pm 0.320$ & HJST \\
\hline 2012 May 6 & 08:08:55.42 & 1053.86729 & $-9.150 \pm 0.122$ & $-13.718 \pm 0.220$ & HJST \\
\hline 2012 May 18 & $07: 35: 21.15$ & 1065.83749 & $-1.843 \pm 0.060$ & $-6.443 \pm 0.108$ & HET \\
\hline 2012 May 20 & 07:37:07.78 & 1067.83880 & $-25.681 \pm 0.030$ & $-30.281 \pm 0.054$ & HET \\
\hline 2012 Jun 5 & $06: 29: 24.15$ & 1083.79236 & $-5.223 \pm 0.080$ & $-9.823 \pm 0.144$ & HET \\
\hline 2012 Jun 26 & 08:03:52.39 & 1104.85862 & $-26.743 \pm 0.086$ & $-31.311 \pm 0.155$ & HJST \\
\hline 2012 Aug 20 & 02:04:42.20 & 1159.5866 & $26.670 \pm 0.080$ & $22.402 \pm 0.144$ & $1.93 \mathrm{~m} \mathrm{OHP}$ \\
\hline 2012 Aug 21 & 01:53:19.7 & 1160.5787 & $34.880 \pm 0.130$ & $30.612 \pm 0.234$ & $1.93 \mathrm{~m} \mathrm{OHP}$ \\
\hline 2012 Aug 22 & 01:10:33.6 & 1161.5490 & $24.220 \pm 0.080$ & $19.952 \pm 0.144$ & $1.93 \mathrm{~m} \mathrm{OHP}$ \\
\hline 2012 Sep 7 & 19:30:08.6 & 1178.3126 & $-19.070 \pm 0.160$ & $-23.339 \pm 0.288$ & $1.93 \mathrm{~m} \mathrm{OHP}$ \\
\hline
\end{tabular}

Notes.

${ }^{\mathrm{a}}$ Uncorrected radial velocities.

${ }^{\mathrm{b}}$ Corrected radial velocities with scaled error bars.

(This table is available in machine-readable form.) 


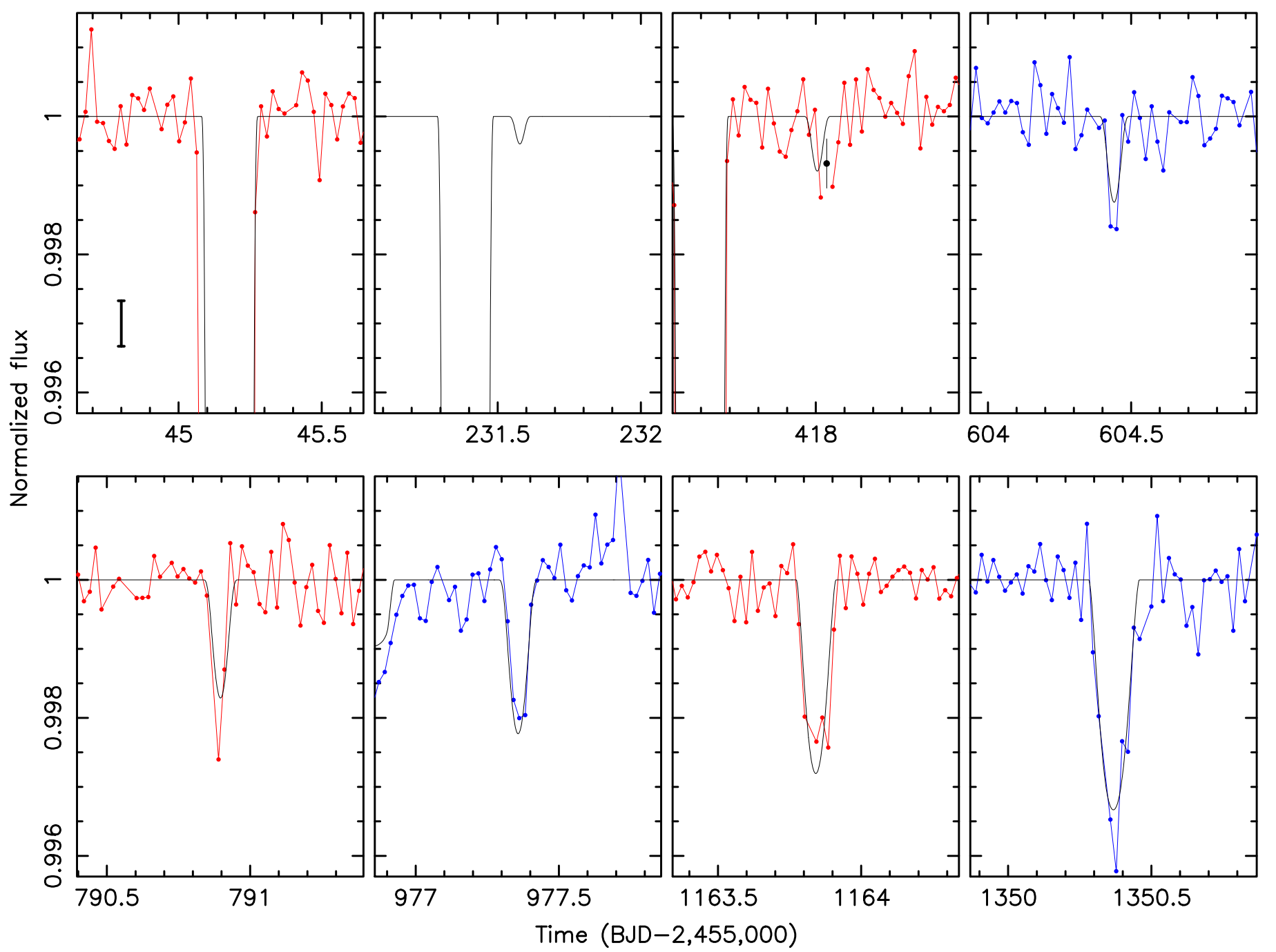

Figure 2. The detrended and normalized Kepler light curves are shown for the six observed transits of the middle planet (across the primary star). The color of the points denotes the observing season: red points are used for Q2, Q6, Q10, and Q14; blue points are used for Q4, Q8, Q12, and Q16. For clarity, the error bars on the points are not shown (a representative error bar is shown in the upper left panel). A total of eight primary transits occurred during the span of the Kepler observations, but one transit with a very small amplitude occurred during a primary eclipse (first panel) and another transit was missed during a gap in the data (second panel). The models for the missed events are shown for completeness. The "orphan" transit from the discovery paper is the one at day 977.4 (a transit of the inner planet across the primary occurred at near day 976.9). The transit near day 418.0 had one point affected by a cosmic ray (data quality flag 8192), so that point was not used in the analysis. The black point with the error bar shows where the cosmic-ray-corrected flux would have been had it not been excluded. The last two transits were observed in short-cadence mode (one-minute sampling), but we show the long-cadence data here for consistency.

use that measurement in our analysis. The uncertainties in the individual measurements in each set were scaled to give $\chi^{2}=N$, where $N=14$ is the number of radial velocity measurements. The offset velocities with the scaled uncertainties are also given in Table 1.

\section{New Transits and Transit Times}

Figure 2 shows the transits of the middle planet across the primary star. During the nominal Kepler mission, this planet transited the primary eight times. However, we were able to observe only six of these transits. The first transit near day 45.15 was blended with an eclipse of the primary, and the second transit near day 231.58 was lost due to a gap in the data. As discussed further below, the primary transits of the middle planet were getting deeper with time, owing to nodal precession of its orbit. The three weak primary transits near days 418.0, 604.5, and 790.9 were not identified until a preliminary orbital solution based on the last three observed primary transits was available. According to our best-fitting model discussed below, the middle planet did not transit the secondary star during the nominal Kepler mission. Even if transits of the secondary star did occur, they would be nearly impossible to see in the data, owing to the faintness of the secondary star.

There were a total of 25 transits of the inner planet Kepler- 47 b across the primary observed in the complete Kepler data set, seven of which are shown here for the first time (see Figure 3). Six primary transits of the inner planet were missed due to gaps in the data. A total of four transits of the outer planet Kepler$47 \mathrm{c}$ across the primary are available in the complete Kepler data set (see Figure 4), and fortunately, none were missed. According to our best-fitting model discussed below, the inner planet had seven transits across the secondary star, and the outer planet had one transit across the secondary star during the nominal Kepler mission. All of these secondary transits had 

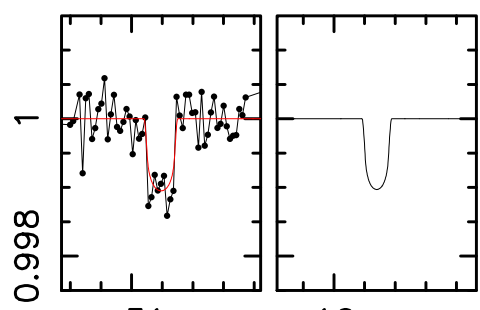

16
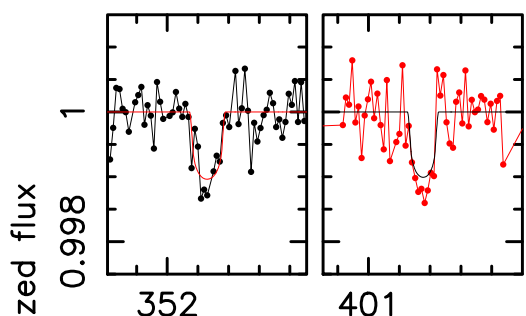

401

\begin{abstract}
448
\end{abstract}
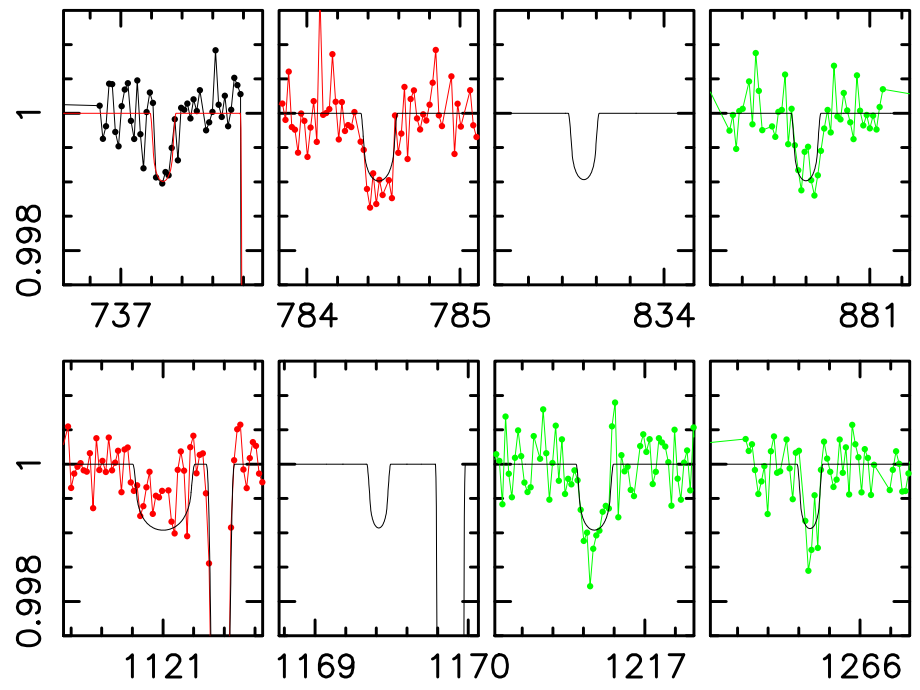

Time (BJD-2,455,000)
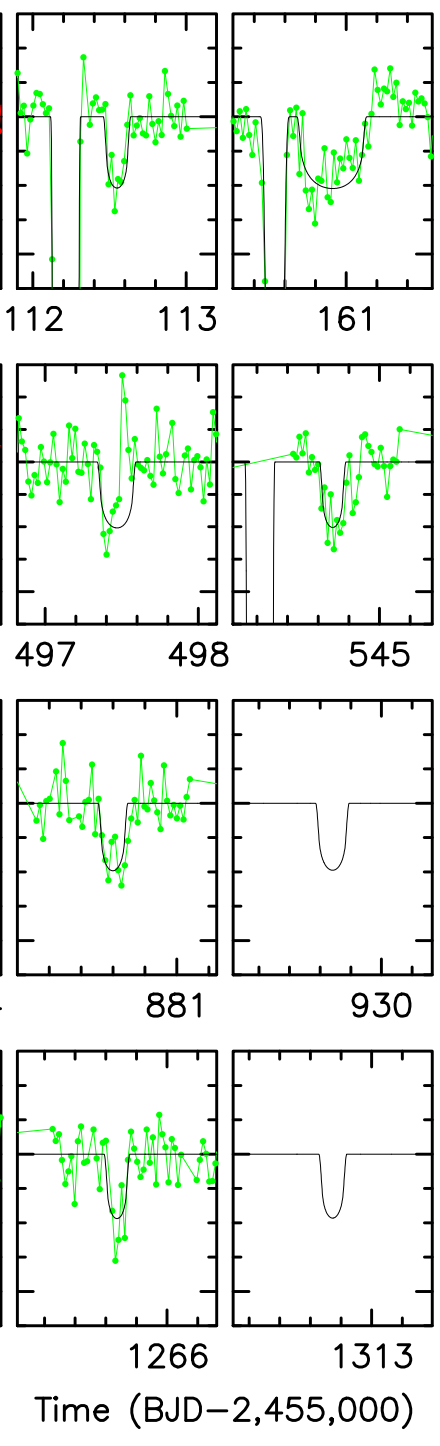
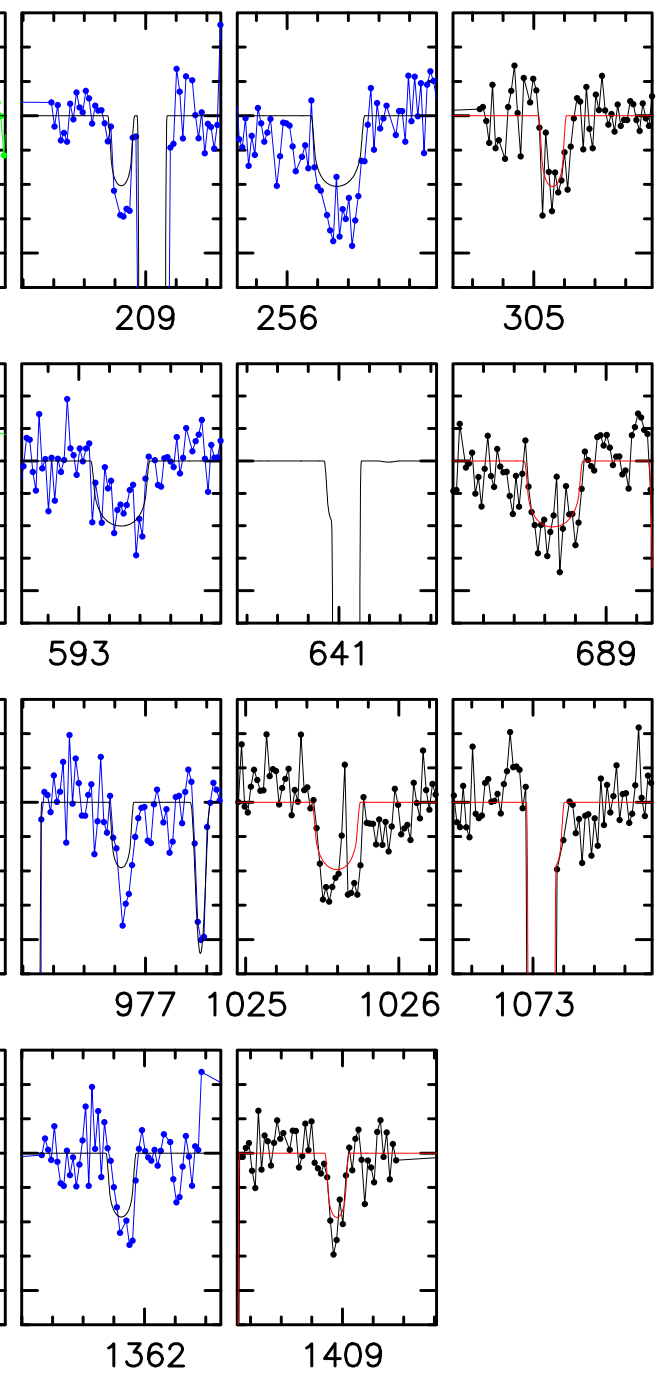

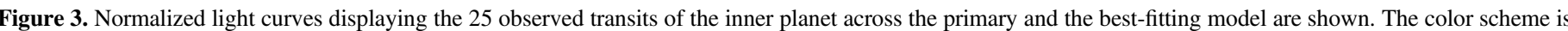

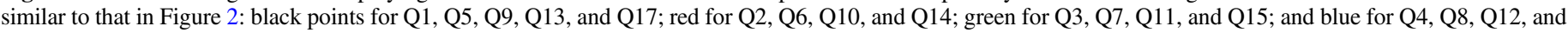

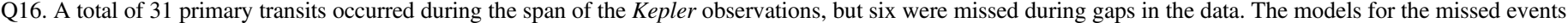

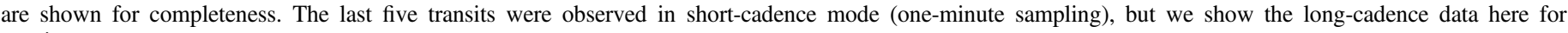
consistency.

impact parameters larger than 0.75 and are not seen above the noise level in the data, owing to the extreme faintness of the secondary star. Hereafter, when we use the term "transit," it is understood to be a transit of a planet across the primary star.

We measured the transit times for each of the planets using the ELC code (Orosz \& Hauschildt 2000). Separate data files were made from the normalized light curve for each event, where between 0.5 and 1.0 days of out-of-eclipse data were kept on either side of the transit event, when possible. In cases where the transit events occurred near eclipses, the eclipses were trimmed from the data.

To model the profile of a planet transit across a single star (assuming a circular orbit), one needs to specify the time of mid-transit, the orbital period, the inclination (or impact parameter), the radius of the star, the ratio of the stellar and planetary radii, and the limb darkening parameters. Given these parameters, the Mandel \& Agol (2002) algorithm can be used to produce a model light curve. Obviously, in the case of
Kepler-47, the primary is part of a binary system and not a single star. Nevertheless, the shapes of the transits can still be matched with a suitable change in the orbital period of the simple model. Because the transit shapes are well-matched, our model of a single planet transiting a single star should produce reliable times of mid-transit.

The model fits to each individual transit were optimized using both a genetic algorithm and a Differential Evolution Monte Carlo Markov Chain (DE-MCMC) routine (Ter Braak 2006). Once a best-fitting model was found, the uncertainties on the observations were scaled to give a reduced $\chi^{2}$ of 1 and the model was re-optimized. The lower $\left(\sigma_{\text {low }}\right)$ and upper $\left(\sigma_{\text {high }}\right) 1 \sigma$ uncertainties were taken to be the interval in the time where $\chi^{2}=\chi_{\min }^{2}+1$ on the low and high sides of the best-fitting time, respectively. Table 2 gives the measured times and durations along with their uncertainties, where the adopted uncertainty on each time was the larger of $\sigma_{\text {low }}$ or $\sigma_{\text {high. }}$. For completeness, we also give the corresponding times and 


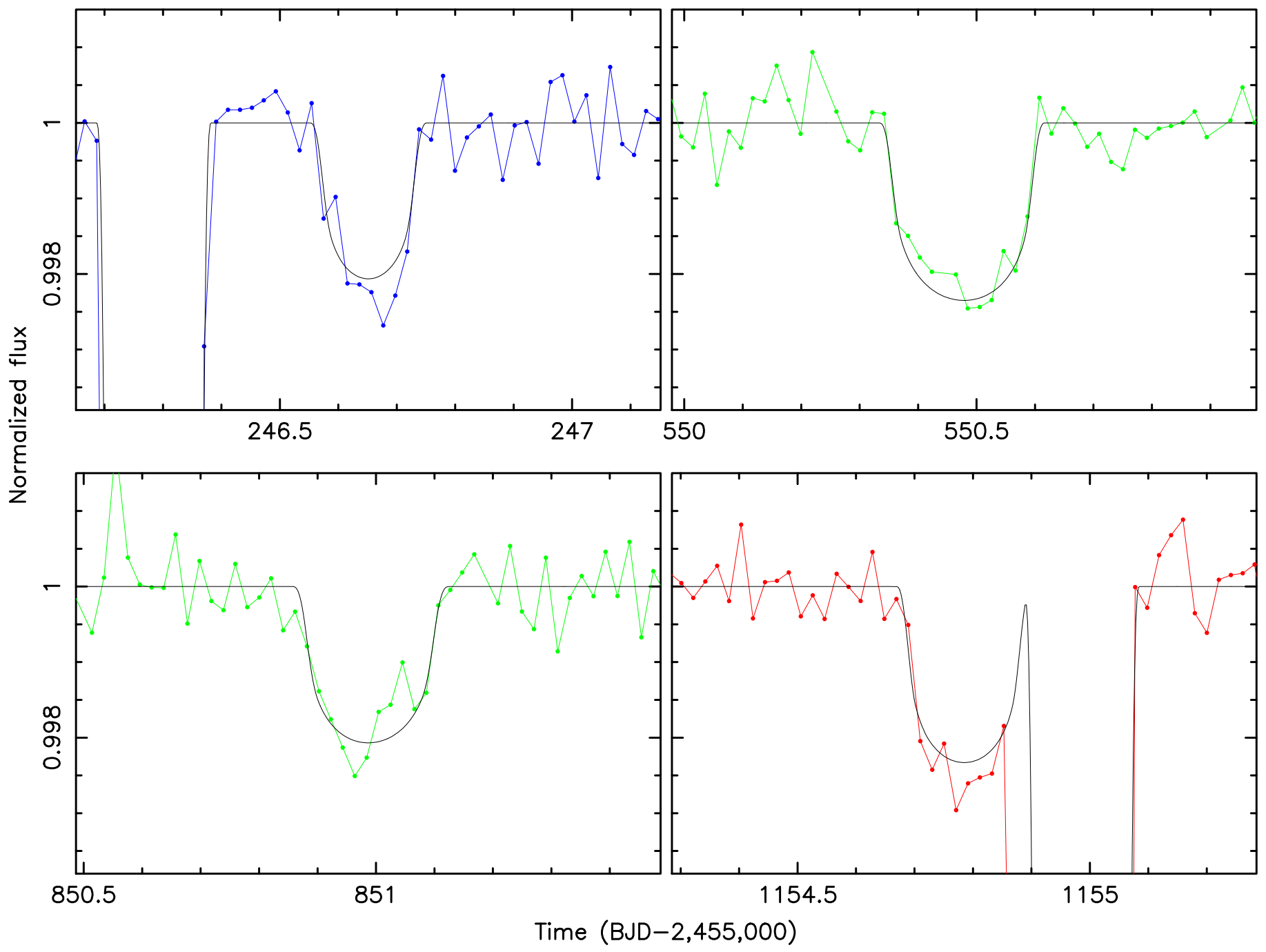

Figure 4. Normalized light curves displaying the four observed transits of the outer planet across the primary on top of the best-fitting model. The color scheme is the same as in Figure 3. The last transit was observed in short-cadence mode (one-minute sampling), but we show the long-cadence data here for consistency.

durations found from the best-fitting photodynamical model described below.

There is a point that was affected by a cosmic ray (data quality flag 8192) close to the middle of the transit of the middle planet, near day 418.0 (see Figure 2). That point was not used in the analysis. When that transit is plotted with all of the points connected by lines and without the bad point, it appears the transit is $\approx 30-45$ minutes late relative to the model. The formal uncertainty on the measured transit time with the bad point excluded is about nine minutes. There is a corrected flux value for the time in question, and the black point with the error bar in Figure 2 shows where that corrected flux would have been had that observation been in the detrended light curve. The transit looks less convincing when that point is shown, because its flux value is a bit higher than the flux values on either side. We did some Monte Carlo simulations where the bad point was put back into the light curve, but with a flux value drawn from a Gaussian distribution centered on the corrected value and with a standard deviation equal to the reported uncertainty. The measured transit time was shifted earlier by about three minutes from where it was without the questionable point included.
As discussed in Orosz et al. (2012b), the planet transit times are not expected to follow a simple linear ephemeris-owing mostly to the motion of the primary star around the system barycenter (because the location of the primary star changes as it orbits, transits can occur early or late relative to a stationary star), and to a lesser extent, perturbations from the binary (Agol et al. 2005). We fitted each set of transit times to linear ephemerides, and formed "Observed minus Computed" $(O-C)$ diagrams that are shown in Figure 5. The middle planet has $O-C$ variations of a few hours, which is strong evidence that the transits are due to a circumbinary body and are not from a background blend (a common false-positive scenario for planets around single stars). The inner planet has maximum $O-C$ variations of about $25 \mathrm{hr}$, and the outer planet has maximum $O-C$ variations of nearly $50 \mathrm{hr}$. Moreover, the $O-C$ variations for all three planets vary cyclically on the binary star's orbit. We define phase 0.0 as the time of the primary eclipse. Given this definition, the $O-C$ variation is zero at phases 0.0 and 0.5 because the projected displacement of the star from the center of mass is at a minimum. Near phases 0.25 and 0.75 , the star is at the projected ends of its orbit, and the timing variations are largest. If the binary system 
Table 2

Times and Durations of Planetary Transits

\begin{tabular}{|c|c|c|c|c|c|c|c|}
\hline Cycle\# & $\begin{array}{l}\text { Measured } \\
\text { Time }^{\mathrm{a}}\end{array}$ & $\begin{array}{c}\text { Uncertainty } \\
\text { (minute) }\end{array}$ & $\begin{array}{l}\text { Duration } \\
\text { (hr) }\end{array}$ & $\begin{array}{l}\text { Model } \\
\text { Time }^{\mathrm{a}}\end{array}$ & $\begin{array}{c}\text { Model } \\
\text { Duration (hr) }\end{array}$ & $\begin{array}{c}\text { Impact } \\
\text { Parameter }\end{array}$ & Note \\
\hline \multicolumn{8}{|c|}{ Inner Planet } \\
\hline$\overline{1.0}$ & -30.80969 & 4.46 & $4.55 \pm 0.19$ & -30.80876 & 4.76 & 0.29 & \\
\hline 2.0 & $\cdots$ & $\cdots$ & $\cdots$ & 16.27666 & 4.10 & 0.30 & Data gap \\
\hline 3.0 & 65.24832 & 22.29 & $5.81 \pm 1.40$ & 65.24587 & 6.41 & 0.29 & \\
\hline 4.0 & 112.54179 & 10.51 & $2.94 \pm 0.69$ & 112.54644 & 3.76 & 0.33 & \\
\hline 5.0 & 160.89953 & 32.75 & $10.83 \pm 2.66$ & 160.90621 & 10.45 & 0.28 & \\
\hline 6.0 & 208.84796 & 7.54 & $3.47 \pm 0.66$ & 208.83932 & 3.69 & 0.36 & \\
\hline 7.0 & 256.34222 & 11.56 & $8.86 \pm 0.69$ & 256.32139 & 7.90 & 0.30 & \\
\hline 8.0 & 305.14101 & 7.61 & $4.97 \pm 0.61$ & 305.12131 & 3.85 & 0.38 & \\
\hline 9.0 & 352.25457 & 12.36 & $5.41 \pm 1.90$ & 352.25805 & 5.10 & 0.35 & \\
\hline 10.0 & 401.35072 & 11.18 & $4.16 \pm 0.50$ & 401.35481 & 4.34 & 0.40 & \\
\hline 11.0 & 448.43586 & 9.83 & $3.16 \pm 0.60$ & 448.43157 & 4.06 & 0.40 & \\
\hline 12.0 & 497.42556 & 12.52 & $2.96 \pm 0.89$ & 497.46945 & 5.54 & 0.41 & \\
\hline 13.0 & 544.73196 & 17.72 & $6.90 \pm 0.82$ & 544.69458 & 3.61 & 0.44 & \\
\hline 14.0 & 593.25486 & 8.75 & $8.75 \pm 0.37$ & 593.27974 & 8.76 & 0.41 & \\
\hline 15.0 & $\cdots$ & $\cdots$ & $\cdots$ & 640.98828 & 3.45 & 0.48 & Data gap \\
\hline 16.0 & 688.66867 & 11.27 & $8.47 \pm 0.47$ & 688.65503 & 8.74 & 0.42 & \\
\hline 17.0 & 737.27699 & 37.31 & $2.99 \pm 1.23$ & 737.27888 & 3.51 & 0.51 & \\
\hline 18.0 & 784.46811 & 19.36 & $4.84 \pm 3.83$ & 784.47271 & 5.25 & 0.47 & \\
\hline 19.0 & $\cdots$ & $\cdots$ & $\cdots$ & 833.53075 & 3.85 & 0.53 & Data gap \\
\hline 20.0 & 880.61567 & 11.00 & $4.90 \pm 0.99$ & 880.60230 & 3.95 & 0.52 & \\
\hline 21.0 & $\cdots$ & $\cdots$ & $\cdots$ & 929.68734 & 4.72 & 0.54 & Corrupted data \\
\hline 22.0 & 976.86285 & 11.31 & $4.08 \pm 0.72$ & 976.84585 & 3.40 & 0.56 & \\
\hline 23.0 & 1025.61184 & 12.69 & $7.25 \pm 0.53$ & 1025.60438 & 7.09 & 0.53 & \\
\hline 24.0 & $\cdots$ & $\cdots$ & $\cdots$ & 1073.13111 & 3.18 & 0.59 & Blend \\
\hline 25.0 & 1120.93797 & 70.76 & $7.42 \pm 2.54$ & 1121.01153 & 9.26 & 0.53 & \\
\hline 26.0 & $\cdots$ & $\cdots$ & $\cdots$ & 1169.41978 & 3.19 & 0.61 & Data gap \\
\hline 27.0 & 1216.66103 & 15.68 & $5.72 \pm 0.84$ & 1216.67569 & 5.38 & 0.57 & \\
\hline 28.0 & 1265.68597 & 20.38 & $2.50 \pm 0.75$ & 1265.67744 & 3.47 & 0.62 & \\
\hline 29.0 & $\ldots$ & $\ldots$ & $\ldots$ & 1312.74767 & 3.85 & 0.60 & Data gap \\
\hline 30.0 & 1361.87205 & 11.00 & $4.14 \pm 1.12$ & 1361.85261 & 4.20 & 0.61 & \\
\hline 31.0 & 1408.96020 & 12.58 & $3.00 \pm 1.37$ & 1408.96429 & 3.25 & 0.63 & \\
\hline \multicolumn{8}{|c|}{ Middle Planet } \\
\hline 1.0 & $\cdots$ & $\cdots$ & $\cdots$ & 45.15444 & 0.90 & 0.99 & Blend \\
\hline 2.0 & $\cdots$ & $\cdots$ & $\cdots$ & 231.57791 & 1.33 & 0.97 & Data gap \\
\hline 3.0 & 418.04185 & 8.93 & $1.71 \pm 0.86$ & 418.00486 & 1.66 & 0.95 & One cadence missing \\
\hline 4.0 & 604.43883 & 5.69 & $1.12 \pm 0.46$ & 604.44003 & 1.97 & 0.94 & \\
\hline 5.0 & 790.88576 & 16.08 & $1.55 \pm 0.71$ & 790.89628 & 2.31 & 0.93 & \\
\hline 6.0 & 977.36103 & 4.98 & $1.86 \pm 0.65$ & 977.35770 & 2.73 & 0.91 & Original orphan \\
\hline 7.0 & 1163.84405 & 3.35 & $2.99 \pm 0.21$ & 1163.84154 & 3.31 & 0.90 & \\
\hline 8.0 & 1350.36851 & 6.08 & $4.81 \pm 0.60$ & 1350.36740 & 4.23 & 0.88 & \\
\hline \multicolumn{8}{|c|}{ Outer Planet } \\
\hline$\overline{1.0}$ & 246.65182 & 5.95 & $4.14 \pm 0.64$ & 246.65033 & 4.29 & 0.70 & \\
\hline 2.0 & 550.47424 & 5.08 & $5.96 \pm 0.39$ & 550.47748 & 6.16 & 0.56 & \\
\hline 3.0 & 850.97978 & 11.50 & $8.07 \pm 1.46$ & 850.98582 & 5.80 & 0.72 & \\
\hline 4.0 & 1154.79184 & 6.42 & $6.15 \pm 0.68$ & 1154.78852 & 4.95 & 0.55 & \\
\hline
\end{tabular}

Note.

${ }^{\mathrm{a}}$ BJD-2,455,000.

(This table is available in machine-readable form.)

and the planet both have circular orbits, then the $O-C$ variation with orbital phase will be sinusoidal; see Figure 5. Note that the transits of the middle planet span a limited range in binary phase due to the roughly 25:1 ratio of the planet's orbital period to the binary orbital period, so only a small portion of the expected sinusoidal variation is seen. This limited range in binary phase has no adverse effect on the mass determination from the photodynamical model discussed below.

Finally, the planet transit durations depend on the relative projected velocities of the primary star and the planet. If a transit occurs near primary eclipse, the bodies are moving in opposite directions, resulting in a narrow (short-duration) transit. For transits near secondary eclipse, the bodies are moving in the same direction, yielding a longer duration transit; see Figure 5. Note that, if the planet transits near the stellar limb rather than the center, then the duration can be short at any phase-this is the case for the middle planet. Kostov et al. (2014) give an analytic expression to compute the transit durations as a function of binary phase, assuming a circular orbit for the planet and a constant impact parameter. Figure 5 shows the model duration curves for each planet, where we 


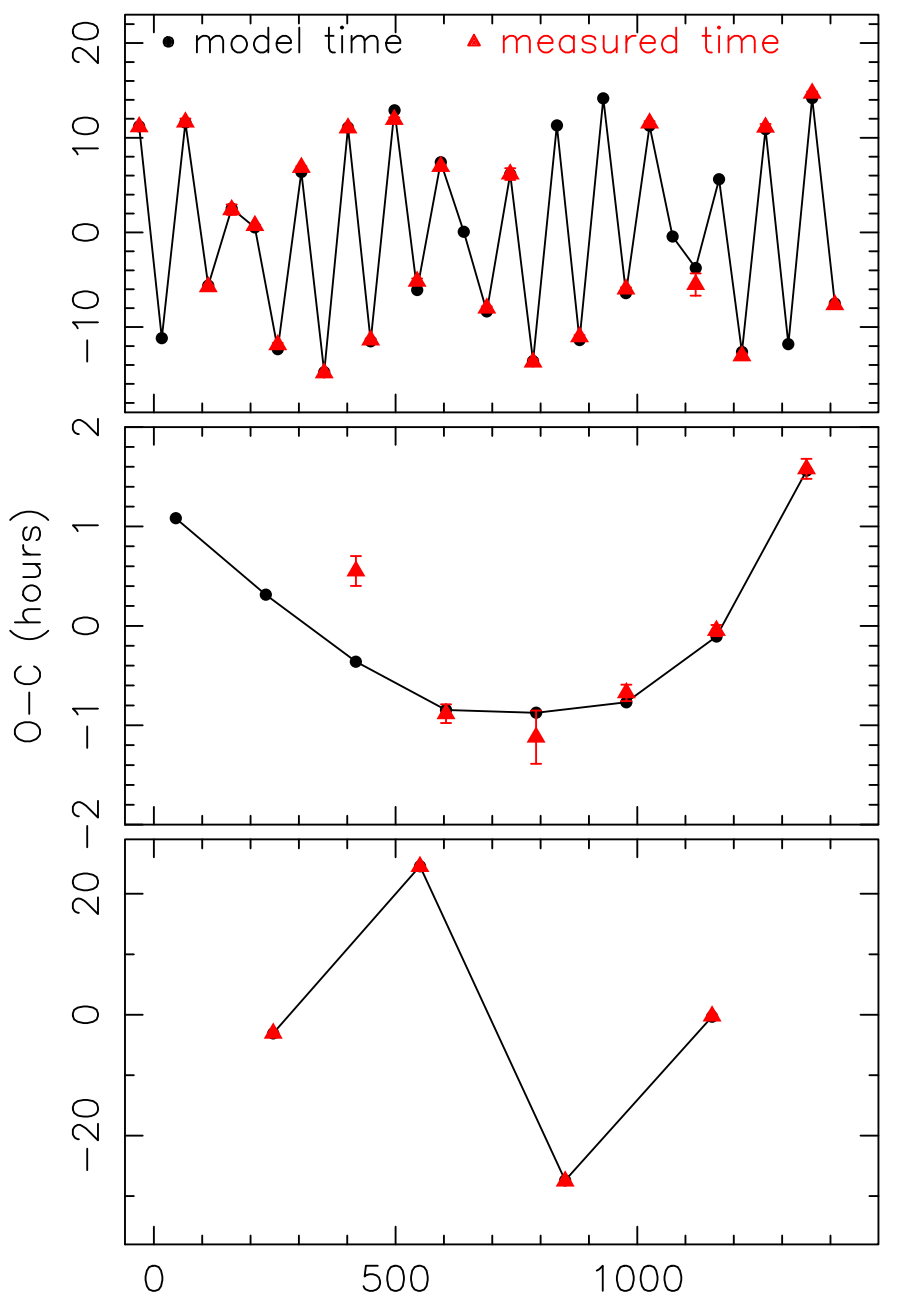

Time (BJD-2,455,000)

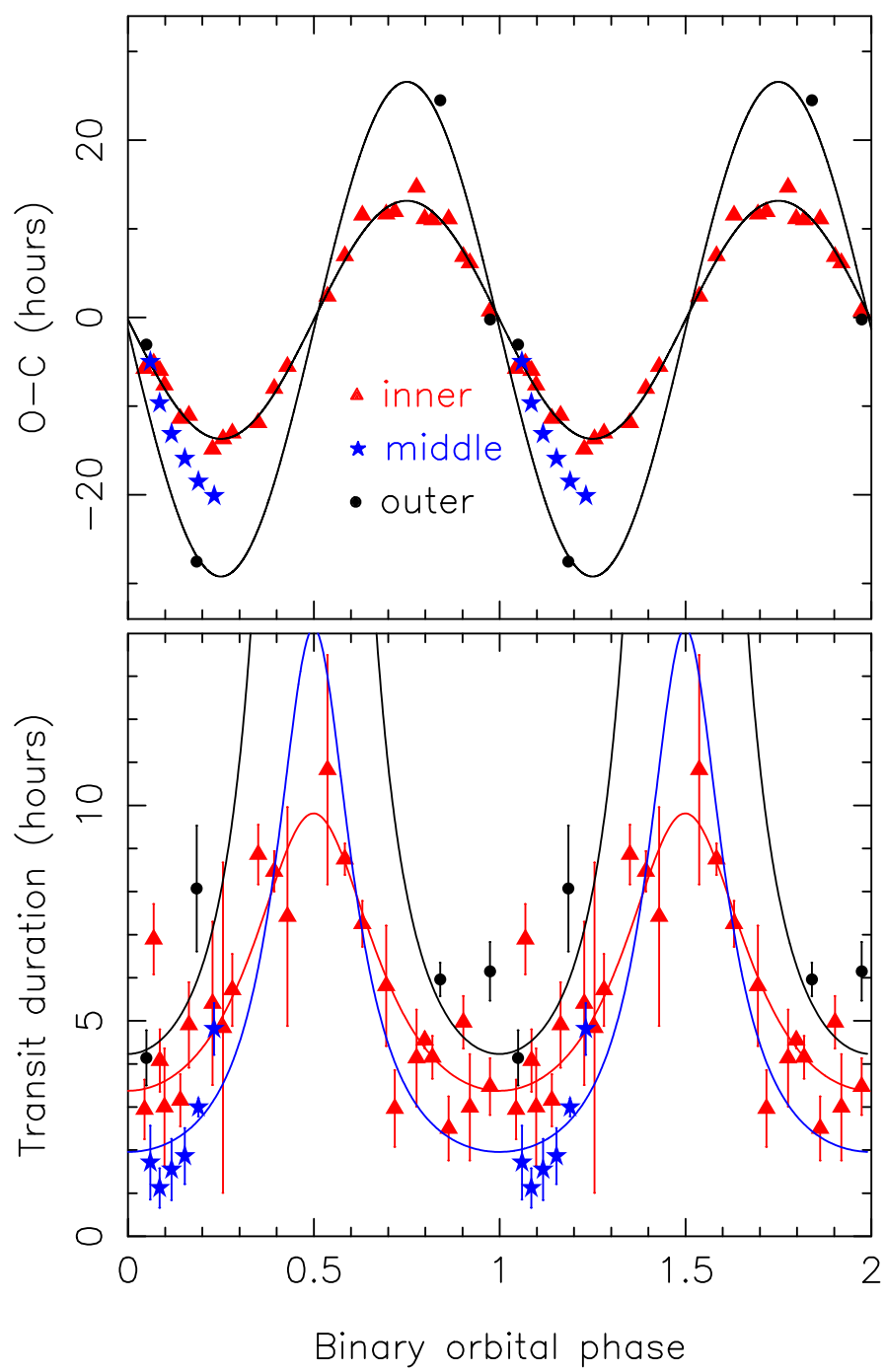

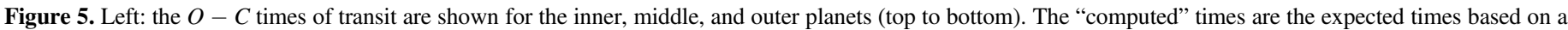

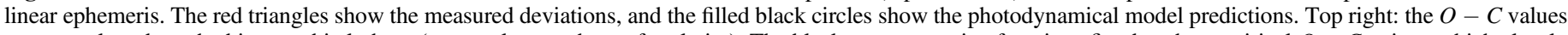

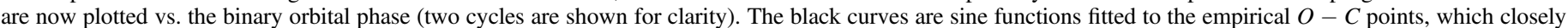

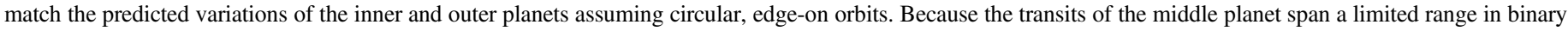

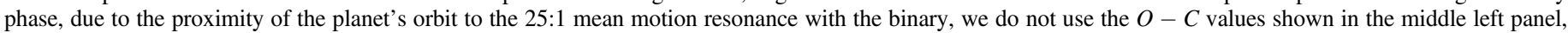

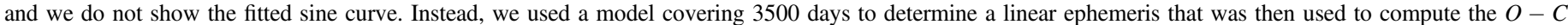

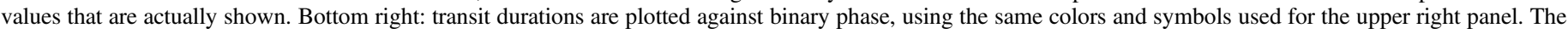

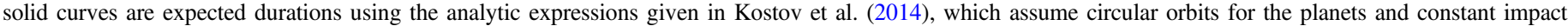
parameters (we used $b=0.45,0.63$, and 0.92 for the inner, middle, and outer planets, respectively).

used $b=0.45,0.63$, and 0.92 for the inner, middle, and outer planets, respectively. The model curves do a reasonable job of fitting the observed durations, although the scatter is somewhat large given that individual transits can have an impact parameter that is quite different than the mean; see Table 2.

\section{Stellar Eclipse Times and Corrections}

We measured the times of the primary and secondary eclipses using the technique outlined in Welsh et al. (2012) and Orosz et al. (2012b), and the results are given in Table 3. Note that the cycle numbers given for the secondary eclipses are not exactly half integers because the orbit is eccentric.

The times of the primary eclipses were fitted to a linear ephemeris. The $O-C$ residual times were computed and are shown in Figure 6. As was the case in our earlier work (Orosz et al. 2012b), there are coherent modulations of up to about two minutes, with a quasiperiod near 178 days. These timing anomalies are caused by star spots on the primary that are partially or fully covered during the primary eclipse. When this occurs, the eclipse profile is not symmetric in time. As discussed in Orosz et al. (2012b) and Welsh et al. (2015), the shift in the measured eclipse time will depend on where the spot is seen on the face of the primary star and when the secondary passes over it. Also, the slope of the light curve near primary eclipse depends on where the spot is on the primary as it rotates into and out of view. Therefore, a correlation is expected between the local light curve slope and the $O-C$ residual (Holczer et al. 2015; Mazeh et al. 2015), which is shown in Figure 7. A linear function was fitted to the data shown in Figure 7 and used to statistically correct the times of the primary eclipses. A new linear ephemeris was fitted to the corrected eclipse times, and the $O-C$ residuals are shown in 
Table 3

Times of Stellar Eclipses

\begin{tabular}{|c|c|c|c|c|c|c|}
\hline Cycle \# & $\begin{array}{l}\text { Primary } \\
\text { Time }^{\mathrm{a}}\end{array}$ & $\begin{array}{l}\text { Corrected } \\
\text { Time }^{\mathrm{a}}\end{array}$ & $\begin{array}{l}\text { Uncertainty } \\
\text { (minutes) }\end{array}$ & Cycle \# & $\begin{array}{l}\text { Secondary } \\
\text { Time }^{\mathrm{a}}\end{array}$ & $\begin{array}{l}\text { Uncertainty } \\
\text { (minutes) }\end{array}$ \\
\hline 0.0 & $\ldots$ & $\ldots$ & $\ldots$ & 0.487385 & -33.12242 & 2.55 \\
\hline 1.0 & -29.30551 & -29.30557 & 0.51 & 1.487385 & -25.67575 & 2.65 \\
\hline 2.0 & -21.85721 & -21.85712 & 0.51 & 2.487385 & -18.22955 & 2.65 \\
\hline 3.0 & $\ldots$ & $\ldots$ & $\ldots$ & 3.487385 & -10.77950 & 2.54 \\
\hline 4.0 & -6.96069 & -6.96058 & 0.49 & 4.487385 & $\ldots$ & $\ldots$ \\
\hline 5.0 & $\ldots$ & $\ldots$ & $\ldots$ & 5.487385 & 4.11925 & 3.00 \\
\hline 6.0 & 7.93602 & 7.93622 & 0.48 & 6.487385 & 11.56766 & 2.89 \\
\hline 7.0 & $\ldots$ & $\ldots$ & $\ldots$ & 7.487385 & 19.01206 & 2.77 \\
\hline 8.0 & 22.83277 & 22.83289 & 0.48 & 8.487385 & 26.46528 & 2.65 \\
\hline 9.0 & 30.28122 & 30.28145 & 0.48 & 9.487385 & 33.91418 & 2.31 \\
\hline 10.0 & 37.72961 & 37.72978 & 0.48 & 10.487385 & 41.36150 & 2.65 \\
\hline 11.0 & $\ldots$ & $\ldots$ & $\ldots$ & 11.487385 & 48.80864 & 2.54 \\
\hline 12.0 & $\ldots$ & $\ldots$ & $\ldots$ & 12.487385 & 56.25788 & 2.77 \\
\hline 13.0 & 60.07493 & 60.07506 & 0.49 & 13.487385 & $\ldots$ & $\ldots$ \\
\hline 14.0 & 67.52346 & 67.52350 & 0.50 & 14.487385 & 71.15074 & 2.42 \\
\hline 15.0 & 74.97166 & 74.97165 & 0.49 & 15.487385 & 78.60188 & 2.65 \\
\hline 16.0 & 82.42033 & 82.42022 & 0.49 & 16.487385 & 86.05079 & 2.54 \\
\hline 17.0 & 89.86868 & 89.86856 & 0.49 & 17.487385 & 93.49712 & 3.12 \\
\hline 18.0 & 97.31711 & 97.31693 & 0.47 & 18.487385 & 100.94813 & 2.54 \\
\hline 19.0 & $\ldots$ & $\ldots$ & $\ldots$ & 19.487385 & 108.39654 & 2.54 \\
\hline 20.0 & 112.21389 & 112.21356 & 0.48 & 20.487385 & 115.84383 & 2.42 \\
\hline 21.0 & 119.66226 & 119.66203 & 0.48 & 21.487385 & $\ldots$ & $\ldots$ \\
\hline 22.0 & 127.11047 & 127.11021 & 0.48 & 22.487385 & 130.73682 & 2.54 \\
\hline 23.0 & 134.55888 & 134.55875 & 0.50 & 23.487385 & $\ldots$ & $\ldots$ \\
\hline 24.0 & 142.00720 & 142.00713 & 0.49 & 24.487385 & 145.63577 & 2.77 \\
\hline 25.0 & 149.45537 & 149.45534 & 0.48 & 25.487385 & $\ldots$ & $\ldots$ \\
\hline 26.0 & 156.90382 & 156.90398 & 0.48 & 26.487385 & 160.53487 & 2.65 \\
\hline 27.0 & 164.35231 & 164.35226 & 0.48 & 27.487385 & 167.98122 & 2.54 \\
\hline 28.0 & 171.80081 & 171.80089 & 0.49 & 28.487385 & $\ldots$ & $\ldots$ \\
\hline 29.0 & 179.24881 & 179.24907 & 0.48 & 29.487385 & $\ldots$ & $\ldots$ \\
\hline 30.0 & 186.69701 & 186.69743 & 0.48 & 30.487385 & 190.32692 & 2.54 \\
\hline 31.0 & 194.14522 & 194.14566 & 0.48 & 31.487385 & 197.77646 & 2.65 \\
\hline 32.0 & 201.59352 & 201.59381 & 0.50 & 32.487385 & 205.22630 & 2.54 \\
\hline 33.0 & $\ldots$ & $\ldots$ & $\ldots$ & 33.487385 & 212.67039 & 3.46 \\
\hline 34.0 & $\ldots$ & $\ldots$ & $\ldots$ & 34.487385 & 220.12045 & 2.42 \\
\hline 35.0 & 223.93909 & 223.93924 & 0.50 & 35.487385 & 227.56808 & 3.46 \\
\hline 36.0 & $\ldots$ & $\ldots$ & $\ldots$ & 36.487385 & 235.01796 & 2.42 \\
\hline 37.0 & 238.83626 & 238.83617 & 0.48 & 37.487385 & 242.46574 & 2.42 \\
\hline 38.0 & 246.28459 & 246.28468 & 0.49 & 38.487385 & 249.91273 & 2.54 \\
\hline 39.0 & 253.73305 & 253.73320 & 0.47 & 39.487385 & 257.36230 & 2.54 \\
\hline 40.0 & 261.18094 & 261.18106 & 0.47 & 40.487385 & 264.81024 & 2.42 \\
\hline 41.0 & 268.62946 & 268.62949 & 0.48 & 41.487385 & $\ldots$ & $\ldots$ \\
\hline 42.0 & $\ldots$ & $\ldots$ & $\ldots$ & 42.487385 & 279.70694 & 2.54 \\
\hline 43.0 & 283.52628 & 283.52618 & 0.49 & 43.487385 & $\ldots$ & $\ldots$ \\
\hline 44.0 & 290.97491 & 290.97467 & 0.49 & 44.487385 & 294.60605 & 2.42 \\
\hline 45.0 & 298.42331 & 298.42300 & 0.48 & 45.487385 & $\ldots$ & $\ldots$ \\
\hline 46.0 & 305.87186 & 305.87149 & 0.49 & 46.487385 & 309.50082 & 2.65 \\
\hline 47.0 & 313.32040 & 313.31982 & 0.48 & 47.487385 & $\ldots$ & $\ldots$ \\
\hline 48.0 & 320.76880 & 320.76819 & 0.49 & 48.487385 & 324.39990 & 2.42 \\
\hline 49.0 & 328.21707 & 328.21655 & 0.48 & 49.487385 & 331.84735 & 2.42 \\
\hline 50.0 & 335.66525 & 335.66498 & 0.49 & 50.487385 & 339.29431 & 2.42 \\
\hline 51.0 & 343.11343 & 343.11331 & 0.48 & 51.487385 & 346.74100 & 2.54 \\
\hline 52.0 & 350.56171 & 350.56155 & 0.50 & 52.487385 & 354.19067 & 2.65 \\
\hline 53.0 & 358.00989 & 358.00983 & 0.49 & 53.487385 & 361.63971 & 2.54 \\
\hline 54.0 & 365.45822 & 365.45825 & 0.48 & 54.487385 & 369.08972 & 2.54 \\
\hline 55.0 & 372.90720 & 372.90741 & 0.63 & 55.487385 & 376.53705 & 2.65 \\
\hline 56.0 & $\ldots$ & $\ldots$ & $\ldots$ & 56.487385 & 383.98596 & 2.54 \\
\hline 57.0 & 387.80347 & 387.80380 & 0.47 & 57.487385 & 391.43390 & 2.65 \\
\hline 58.0 & 395.25180 & 395.25220 & 0.48 & 58.487385 & 398.88248 & 2.65 \\
\hline 59.0 & 402.69992 & 402.70035 & 0.50 & 59.487385 & 406.33072 & 2.77 \\
\hline 60.0 & 410.14825 & 410.14865 & 0.49 & 60.487385 & $\ldots$ & $\ldots$ \\
\hline 61.0 & 417.59674 & 417.59717 & 0.49 & 61.487385 & $\ldots$ & $\ldots$ \\
\hline 62.0 & 425.04520 & 425.04559 & 0.50 & 62.487385 & 428.67667 & 2.42 \\
\hline
\end{tabular}


Table 3

(Continued)

\begin{tabular}{|c|c|c|c|c|c|c|}
\hline Cycle \# & $\begin{array}{c}\text { Primary } \\
\text { Time }^{\mathrm{a}}\end{array}$ & $\begin{array}{l}\text { Corrected } \\
\text { Time }^{\mathrm{a}}\end{array}$ & $\begin{array}{l}\text { Uncertainty } \\
\text { (minutes) }\end{array}$ & Cycle \# & $\begin{array}{l}\text { Secondary } \\
\text { Time }^{\mathrm{a}}\end{array}$ & $\begin{array}{l}\text { Uncertainty } \\
\text { (minutes) }\end{array}$ \\
\hline 63.0 & 432.49371 & 432.49371 & 0.92 & 63.487385 & 436.12863 & 3.70 \\
\hline 64.0 & 439.94229 & 439.94229 & 0.48 & 64.487389 & 443.57419 & 2.54 \\
\hline 65.0 & 447.39066 & 447.39035 & 0.49 & 65.487389 & 451.01895 & 2.65 \\
\hline 66.0 & 454.83932 & 454.83868 & 0.50 & 66.487389 & 458.47025 & 2.65 \\
\hline 67.0 & $\ldots$ & $\ldots$ & $\ldots$ & 67.487389 & $\ldots$ & $\ldots$ \\
\hline 68.0 & 469.73666 & 469.73569 & 0.51 & 68.487389 & 473.36713 & 2.42 \\
\hline 69.0 & $\ldots$ & $\ldots$ & $\ldots$ & 69.487389 & 480.81107 & 2.54 \\
\hline 70.0 & 484.63339 & 484.63257 & 0.50 & 70.487389 & 488.26462 & 2.54 \\
\hline 71.0 & $\ldots$ & $\ldots$ & $\ldots$ & 71.487389 & 495.71335 & 2.42 \\
\hline 72.0 & $\cdots$ & $\ldots$ & $\ldots$ & 72.487389 & 503.15891 & 2.65 \\
\hline 73.0 & 506.97739 & 506.97763 & 0.49 & 73.487389 & 510.60684 & 2.77 \\
\hline 74.0 & 514.42554 & 514.42584 & 0.49 & 74.487389 & 518.05542 & 2.77 \\
\hline 75.0 & 521.87421 & 521.87439 & 0.47 & 75.487389 & 525.50653 & 2.54 \\
\hline 76.0 & 529.32257 & 529.32281 & 0.48 & 76.487389 & 532.95557 & 2.65 \\
\hline 77.0 & 536.77094 & 536.77118 & 0.47 & 77.487389 & 540.40015 & 2.54 \\
\hline 78.0 & $\ldots$ & $\ldots$ & $\ldots$ & 78.487389 & 547.84821 & 2.77 \\
\hline 79.0 & 551.66760 & 551.66797 & 0.48 & 79.487389 & $\ldots$ & $\ldots$ \\
\hline 80.0 & $\ldots$ & $\ldots$ & $\ldots$ & 80.487389 & $\ldots$ & $\ldots$ \\
\hline 81.0 & $\ldots$ & $\ldots$ & $\ldots$ & 81.487389 & $\ldots$ & $\ldots$ \\
\hline 82.0 & 574.01306 & 574.01324 & 0.48 & 82.487389 & 577.64178 & 2.65 \\
\hline 83.0 & 581.46143 & 581.46149 & 0.48 & 83.487389 & $\ldots$ & $\cdots$ \\
\hline 84.0 & $\cdots$ & $\ldots$ & $\ldots$ & 84.487389 & 592.54077 & 2.54 \\
\hline 85.0 & $\ldots$ & $\ldots$ & $\ldots$ & 85.487389 & 599.98920 & 2.42 \\
\hline 86.0 & 603.80609 & 603.80603 & 0.54 & 86.487389 & 607.43634 & 2.54 \\
\hline 87.0 & 611.25482 & 611.25470 & 0.52 & 87.487389 & 614.88654 & 2.42 \\
\hline 88.0 & 618.70325 & 618.70300 & 0.52 & 88.487389 & 622.33221 & 2.65 \\
\hline 89.0 & 626.15149 & 626.15137 & 0.52 & 89.487389 & 629.78101 & 2.77 \\
\hline 90.0 & 633.60010 & 633.60004 & 0.51 & 90.487389 & $\ldots$ & $\ldots$ \\
\hline 91.0 & $\ldots$ & $\ldots$ & $\ldots$ & 91.487389 & 644.67877 & 2.54 \\
\hline 92.0 & 648.49695 & 648.49652 & 0.47 & 92.487389 & 652.12604 & 2.54 \\
\hline 93.0 & 655.94531 & 655.94482 & 0.47 & 93.487389 & 659.57526 & 2.54 \\
\hline 94.0 & 663.39392 & 663.39337 & 0.48 & 94.487389 & 667.02484 & 2.42 \\
\hline 95.0 & 670.84247 & 670.84210 & 0.48 & 95.487389 & 674.47101 & 2.54 \\
\hline 96.0 & $\ldots$ & $\ldots$ & $\ldots$ & 96.487389 & 681.92090 & 2.54 \\
\hline 97.0 & 685.73883 & 685.73865 & 0.48 & 97.487389 & 689.37140 & 2.65 \\
\hline 98.0 & 693.18701 & 693.18695 & 0.49 & 98.487389 & 696.81665 & 2.31 \\
\hline 99.0 & 700.63531 & 700.63531 & 0.49 & 99.487389 & 704.26617 & 2.54 \\
\hline 100.0 & 708.08374 & 708.08374 & 0.49 & 100.487389 & 711.71442 & 2.42 \\
\hline 101.0 & 715.53192 & 715.53204 & 0.48 & 101.487389 & 719.16046 & 2.42 \\
\hline 102.0 & 722.98016 & 722.98029 & 0.48 & 102.487389 & 726.60980 & 2.42 \\
\hline 103.0 & 730.42853 & 730.42883 & 0.47 & 103.487389 & 734.05872 & 2.54 \\
\hline 104.0 & 737.87714 & 737.87732 & 0.47 & 104.487389 & 741.50854 & 2.42 \\
\hline 105.0 & 745.32550 & 745.32550 & 0.48 & 105.487389 & 748.95648 & 2.54 \\
\hline 106.0 & 752.77393 & 752.77380 & 0.47 & 106.487389 & 756.40527 & 2.65 \\
\hline 107.0 & $\ldots$ & $\ldots$ & $\ldots$ & 107.487389 & 763.85193 & 2.65 \\
\hline 108.0 & 767.67059 & 767.67065 & 0.47 & 108.487389 & 771.30164 & 2.65 \\
\hline 109.0 & 775.11890 & 775.11896 & 0.48 & 109.487389 & $\ldots$ & $\ldots$ \\
\hline 110.0 & 782.56732 & 782.56732 & 0.51 & 110.487389 & 786.19946 & 2.65 \\
\hline 111.0 & $\ldots$ & $\ldots$ & $\ldots$ & 111.487389 & $\ldots$ & $\ldots$ \\
\hline 112.0 & 797.46442 & 797.46448 & 0.49 & 112.487389 & 801.09283 & 2.54 \\
\hline 113.0 & 804.91309 & 804.91296 & 0.50 & 113.487389 & 808.54272 & 2.42 \\
\hline 114.0 & 812.36139 & 812.36102 & 0.47 & 114.487389 & 815.99078 & 2.77 \\
\hline 115.0 & 819.80957 & 819.80927 & 0.47 & 115.487389 & 823.43982 & 2.54 \\
\hline 116.0 & 827.25800 & 827.25775 & 0.47 & 116.487389 & 830.88733 & 2.65 \\
\hline 117.0 & 834.70618 & 834.70618 & 0.48 & 117.487389 & 838.33765 & 2.65 \\
\hline 118.0 & $\ldots$ & $\ldots$ & $\ldots$ & 118.487389 & $\ldots$ & $\ldots$ \\
\hline 119.0 & 849.60260 & 849.60272 & 0.47 & 119.487389 & 853.23230 & 2.42 \\
\hline 120.0 & 857.05133 & 857.05127 & 0.49 & 120.487389 & 860.68353 & 2.42 \\
\hline 121.0 & 864.49988 & 864.49969 & 0.49 & 121.487389 & 868.13147 & 2.42 \\
\hline 122.0 & 871.94800 & 871.94781 & 0.47 & 122.487389 & 875.57574 & 2.54 \\
\hline 123.0 & 879.39630 & 879.39630 & 0.47 & 123.487389 & 883.02753 & 2.54 \\
\hline 124.0 & 886.84467 & 886.84460 & 0.47 & 124.487389 & 890.47528 & 2.31 \\
\hline 125.0 & 894.29315 & 894.29303 & 0.47 & 125.487389 & 897.92639 & 2.42 \\
\hline
\end{tabular}


Table 3

(Continued)

\begin{tabular}{|c|c|c|c|c|c|c|}
\hline Cycle \# & $\begin{array}{l}\text { Primary } \\
\text { Time }^{\mathrm{a}}\end{array}$ & $\begin{array}{l}\text { Corrected } \\
\text { Time }^{\mathrm{a}}\end{array}$ & $\begin{array}{l}\text { Uncertainty } \\
\text { (minutes) }\end{array}$ & Cycle \# & $\begin{array}{l}\text { Secondary } \\
\text { Time }^{\mathrm{a}}\end{array}$ & $\begin{array}{c}\text { Uncertainty } \\
\text { (minutes) }\end{array}$ \\
\hline 126.0 & 901.74152 & 901.74146 & 0.48 & 126.487389 & $\ldots$ & $\ldots$ \\
\hline 127.0 & 909.19000 & 909.19006 & 0.48 & 127.487389 & 912.82153 & 2.77 \\
\hline 128.0 & 916.63831 & 916.63855 & 0.49 & 128.487381 & 920.26917 & 2.54 \\
\hline 129.0 & 924.08630 & 924.08685 & 0.48 & 129.487381 & $\ldots$ & $\ldots$ \\
\hline 130.0 & $\ldots$ & $\ldots$ & $\ldots$ & 130.487381 & 935.16748 & 2.54 \\
\hline 131.0 & 938.98212 & 938.98297 & 0.53 & 131.487381 & 942.61377 & 2.42 \\
\hline 132.0 & $\ldots$ & $\ldots$ & $\ldots$ & 132.487381 & $\ldots$ & $\ldots$ \\
\hline 133.0 & 953.87927 & 953.87964 & 0.55 & 133.487381 & $\ldots$ & $\ldots$ \\
\hline 134.0 & 961.32800 & 961.32831 & 0.57 & 134.487381 & 964.95990 & 3.12 \\
\hline 135.0 & 968.77692 & 968.77704 & 0.57 & 135.487381 & 972.40747 & 2.42 \\
\hline 136.0 & 976.22607 & 976.22589 & 0.58 & 136.487381 & 979.85541 & 2.65 \\
\hline 137.0 & 983.67474 & 983.67450 & 0.52 & 137.487381 & $\ldots$ & $\ldots$ \\
\hline 138.0 & 991.12311 & 991.12274 & 0.51 & 138.487381 & $\ldots$ & $\ldots$ \\
\hline 139.0 & $\ldots$ & $\ldots$ & $\ldots$ & 139.487381 & 1002.20215 & 2.31 \\
\hline 140.0 & 1006.01910 & 1006.01849 & 0.51 & 140.487381 & $\ldots$ & $\ldots$ \\
\hline 141.0 & $\ldots$ & $\ldots$ & $\ldots$ & 141.487381 & 1017.09631 & 2.42 \\
\hline 142.0 & $\ldots$ & $\ldots$ & $\ldots$ & 142.487381 & 1024.54651 & 2.54 \\
\hline 143.0 & 1028.36389 & 1028.36365 & 0.49 & 143.487381 & 1031.99353 & 2.42 \\
\hline 144.0 & 1035.81226 & 1035.81226 & 0.48 & 144.487381 & 1039.44019 & 2.54 \\
\hline 145.0 & 1043.26074 & 1043.26074 & 0.49 & 145.487381 & 1046.89429 & 3.23 \\
\hline 146.0 & $\ldots$ & $\ldots$ & $\ldots$ & 146.487381 & 1054.33911 & 2.65 \\
\hline 147.0 & 1058.15735 & 1058.15723 & 0.48 & 147.487381 & $\ldots$ & $\ldots$ \\
\hline 148.0 & 1065.60583 & 1065.60571 & 0.49 & 148.487381 & 1069.23608 & 2.54 \\
\hline 149.0 & 1073.05432 & 1073.05444 & 0.50 & 149.487381 & 1076.68445 & 2.31 \\
\hline 150.0 & 1080.50232 & 1080.50269 & 0.50 & 150.487381 & 1084.13232 & 2.42 \\
\hline 151.0 & 1087.95056 & 1087.95093 & 0.50 & 151.487381 & 1091.58301 & 2.54 \\
\hline 152.0 & 1095.39880 & 1095.39929 & 0.50 & 152.487381 & 1099.02747 & 2.54 \\
\hline 153.0 & 1102.84717 & 1102.84753 & 0.48 & 153.487381 & $\ldots$ & $\ldots$ \\
\hline 154.0 & 1110.29541 & 1110.29578 & 0.47 & 154.487381 & 1113.92444 & 2.43 \\
\hline 155.0 & $\ldots$ & $\ldots$ & $\ldots$ & 155.487381 & 1121.37476 & 2.32 \\
\hline 156.0 & $\cdots$ & $\cdots$ & $\ldots$ & 156.487381 & $\ldots$ & $\ldots$ \\
\hline 157.0 & 1132.64062 & 1132.64111 & 0.47 & 157.487381 & 1136.27185 & 2.43 \\
\hline 158.0 & $\ldots$ & $\ldots$ & $\ldots$ & 158.487381 & 1143.71802 & 2.32 \\
\hline 159.0 & $\ldots$ & $\ldots$ & $\ldots$ & 159.487381 & 1151.16760 & 2.21 \\
\hline 160.0 & $\ldots$ & $\ldots$ & $\ldots$ & 160.487381 & 1158.61731 & 2.43 \\
\hline 161.0 & 1162.43567 & 1162.43506 & 0.47 & 161.487381 & 1166.06384 & 2.55 \\
\hline 162.0 & $\ldots$ & $\ldots$ & $\ldots$ & 162.487381 & 1173.51453 & 2.21 \\
\hline 163.0 & $\ldots$ & $\ldots$ & $\ldots$ & 163.487381 & 1180.96008 & 2.32 \\
\hline 164.0 & 1184.78064 & 1184.77991 & 0.47 & 164.487381 & 1188.41089 & 2.43 \\
\hline 165.0 & 1192.22864 & 1192.22791 & 0.47 & 165.487381 & 1195.85779 & 2.43 \\
\hline 166.0 & 1199.67688 & 1199.67639 & 0.47 & 166.487381 & 1203.30750 & 2.43 \\
\hline 167.0 & 1207.12500 & 1207.12476 & 0.47 & 167.487381 & 1210.75415 & 2.32 \\
\hline 168.0 & $\ldots$ & $\ldots$ & $\ldots$ & 168.487381 & 1218.20361 & 2.43 \\
\hline 169.0 & $\ldots$ & $\ldots$ & $\ldots$ & 169.487381 & 1225.65259 & 2.21 \\
\hline 170.0 & $\ldots$ & $\ldots$ & $\ldots$ & 170.487381 & 1233.09839 & 2.32 \\
\hline 171.0 & $\ldots$ & $\ldots$ & $\ldots$ & 171.487381 & 1240.54895 & 2.55 \\
\hline 172.0 & 1244.36633 & 1244.36682 & 0.47 & 172.487381 & $\ldots$ & $\ldots$ \\
\hline 173.0 & $\ldots$ & $\ldots$ & $\ldots$ & 173.487381 & 1255.44543 & 2.32 \\
\hline 174.0 & 1259.26306 & 1259.26343 & 0.47 & 174.487381 & 1262.89319 & 2.66 \\
\hline 175.0 & 1266.71155 & 1266.71179 & 0.47 & 175.487381 & 1270.33948 & 2.21 \\
\hline 176.0 & 1274.15991 & 1274.16028 & 0.47 & 176.487381 & 1277.78967 & 2.32 \\
\hline 177.0 & 1281.60815 & 1281.60840 & 0.47 & 177.487381 & 1285.24048 & 2.55 \\
\hline 178.0 & 1289.05664 & 1289.05701 & 0.47 & 178.487381 & 1292.68555 & 2.21 \\
\hline 179.0 & 1296.50476 & 1296.50513 & 0.47 & 179.487381 & 1300.13538 & 2.21 \\
\hline 180.0 & $\ldots$ & $\ldots$ & $\ldots$ & 180.487381 & 1307.58350 & 2.32 \\
\hline 181.0 & $\ldots$ & $\ldots$ & $\ldots$ & 181.487381 & $\ldots$ & $\ldots$ \\
\hline 182.0 & $\ldots$ & $\ldots$ & $\ldots$ & 182.487381 & 1322.48193 & 2.43 \\
\hline 183.0 & 1326.29883 & 1326.29883 & 0.47 & 183.487381 & 1329.93335 & 2.43 \\
\hline 184.0 & $\ldots$ & $\ldots$ & $\ldots$ & 184.487381 & $\ldots$ & $\ldots$ \\
\hline 185.0 & 1341.19531 & 1341.19568 & 0.47 & 185.487381 & 1344.82397 & 2.43 \\
\hline 186.0 & 1348.64368 & 1348.64404 & 0.47 & 186.487381 & 1352.27368 & 2.43 \\
\hline 187.0 & 1356.09229 & 1356.09241 & 0.47 & 187.487381 & 1359.72375 & 2.43 \\
\hline 188.0 & 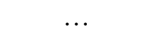 & $\ldots$ & $\ldots$ & 188.487381 & 1367.17090 & 2.32 \\
\hline
\end{tabular}


Table 3

(Continued)

\begin{tabular}{|c|c|c|c|c|c|c|}
\hline Cycle \# & $\begin{array}{l}\text { Primary } \\
\text { Time }^{\mathrm{a}}\end{array}$ & $\begin{array}{l}\text { Corrected } \\
\text { Time }^{\mathrm{a}}\end{array}$ & $\begin{array}{l}\text { Uncertainty } \\
\text { (minutes) }\end{array}$ & Cycle \# & $\begin{array}{c}\text { Secondary } \\
\text { Time }^{\mathrm{a}}\end{array}$ & $\begin{array}{c}\text { Uncertainty } \\
\text { (minutes) }\end{array}$ \\
\hline 189.0 & 1370.98950 & 1370.98914 & 0.47 & 189.487381 & 1374.61914 & 2.32 \\
\hline 190.0 & 1378.43774 & 1378.43726 & 0.47 & 190.487381 & 1382.06519 & 2.66 \\
\hline 191.0 & $\ldots$ & $\ldots$ & $\ldots$ & 191.487381 & 1389.51453 & 2.21 \\
\hline 192.0 & 1393.33435 & 1393.33398 & 0.47 & 192.487381 & 1396.96460 & 2.21 \\
\hline 193.0 & 1400.78259 & 1400.78247 & 0.47 & 193.487381 & 1404.41370 & 2.21 \\
\hline 194.0 & 1408.23083 & 1408.23096 & 0.47 & 194.487381 & 1411.86157 & 2.21 \\
\hline 195.0 & $\ldots$ & $\ldots$ & $\ldots$ & $\ldots$ & $\ldots$ & $\ldots$ \\
\hline 196.0 & 1423.12757 & 1423.12767 & 0.47 & $\ldots$ & $\ldots$ & $\ldots$ \\
\hline
\end{tabular}

Note.

${ }^{\mathrm{a}}$ BJD-2,455,000.

(This table is available in machine-readable form.)

the middle panel of Figure 6. Apart from a feature near day 1000 , the scatter in the residuals has been greatly reduced compared to the the residuals shown in the top panel of the figure. No spot-induced variations were seen in the times of the secondary eclipse, so no correction to those times was applied.

The best-fitting ephemerides for the corrected primary eclipse times and the secondary eclipse times are:

$$
\begin{aligned}
& P_{A}=7.44837568 \pm 0.00000029 \text { day Kepler-47 primary } \\
& P_{B}=7.44837596 \pm 0.00000193 \text { day Kepler-47 secondary } \\
& T_{0}(A)=\text { BJD 2,454,963.246137 } \pm 0.000032 \text { Kepler-47 primary } \\
& T_{0}(B)=\text { BJD 2,454,959.427964 } \pm 0.000228 \quad \text { Kepler-47 secondary }
\end{aligned}
$$

The difference between the primary and secondary periods is $0.02 \pm 0.17 \mathrm{~s}$. The fitted period from the secondary eclipses is formally longer than the fitted period from the primary eclipses, although the difference is not significant. This lack of a significant difference between the two periods means the effect of the planets on the eclipse timings is unmeasurable, and the masses of the planets are constrained by planet-planet interactions, not planet-binary interactions (see Section 6.1).

\section{Photodynamical Modeling of the Light and Velocity Curves}

The complete Kepler-47 light and velocity curves were modeled using the ELC code. Given five bodies with some initial positions and velocities, the Newtonian equations of motion can be integrated, thereby giving the positions and velocities of each body as a function of time. Then, given the position of each body on the plane of the sky at a specific time (corrected for light travel time) and information about their radiative properties, the light curve can be computed. In the discussion below, we give details of the photodynamical model and its application to the Kepler-47 data.

\subsection{ODE Integrator}

The numerical integrator that ELC uses is a symplectic, 12th-order Gaussian Runge-Kutta (GRK) routine based on methods and codes devised by Hairer et al. (2006). For situations where only the mutual gravitational forces between the five bodies are considered, the ordinary differential equations (ODEs) are second-order and can be written such that only the positions of the bodies appear explicitly. GRK is a collocation method in which a system of equations based on the Gaussian quadrature nodes must be solved at each time step. In particular, for the 12th-order method, there are six nodes that determine the coefficients of the collocation polynomial. These coefficients are solved for iteratively. With a suitable initial guess, the convergence of this iteration is quadratic-and hence, very fast. If needed, the equations of motion can be modified to account for extra effects such as precession due to General Relativity (GR) and/or precession due to tidal bulges on the stars (Eggleton et al. 1998; Mardling \& Lin 2002). In the case of the GR correction, the velocities of the bodies appear explicitly in the equations of motion, and the first-order ODEs must be solved using a different iteration scheme. This iteration scheme is only to first order, so the convergence is somewhat slower than the second-order scheme.

Many of the symplectic integrators used in the field of solar system dynamics (e.g., Wisdom \& Holman 1991) rely on a factorization (or splitting) of the Hamiltonian in a Lie algebra sense. These integrators are very fast, but are best applied to problems where there is one dominant central mass. One advantage of GRK integrators is that they do not approach the issue as a Lie algebra factorization and hence do not have the restriction of just one large mass. The trade-off is that the step size is determined by the shortest period, which results in longer run-times (we typically use a time step $h$ that is about 400 times smaller than the smallest period in the system). However, we note that, even with the relatively low speed compared to other integrators, it takes much less than one second to solve the coupled equations of motion for the five bodies in Kepler-47 over a 1500 day time span using the GRK method.

The GRK scheme does have another advantage, in that it is possible to find positions and velocities at intermediate times to high accuracy with relatively few computations. In our implementation, the solutions at the six internal nodal points used for the collocation for each time step are saved. Using the collocation points with divided differences, it is easy to determine the positions and velocities of each body at intermediate times to the same order of accuracy (namely, 12 th) as at the integration times (e.g., $t_{\text {start }}, t_{\text {start }}+h, t_{\text {start }}+2 h$, etc.). The number of operations needed to achieve the 12thorder accuracy is comparable to what one would need for a spline interpolation scheme, which would give the intermediate values with a much lower-order accuracy. 


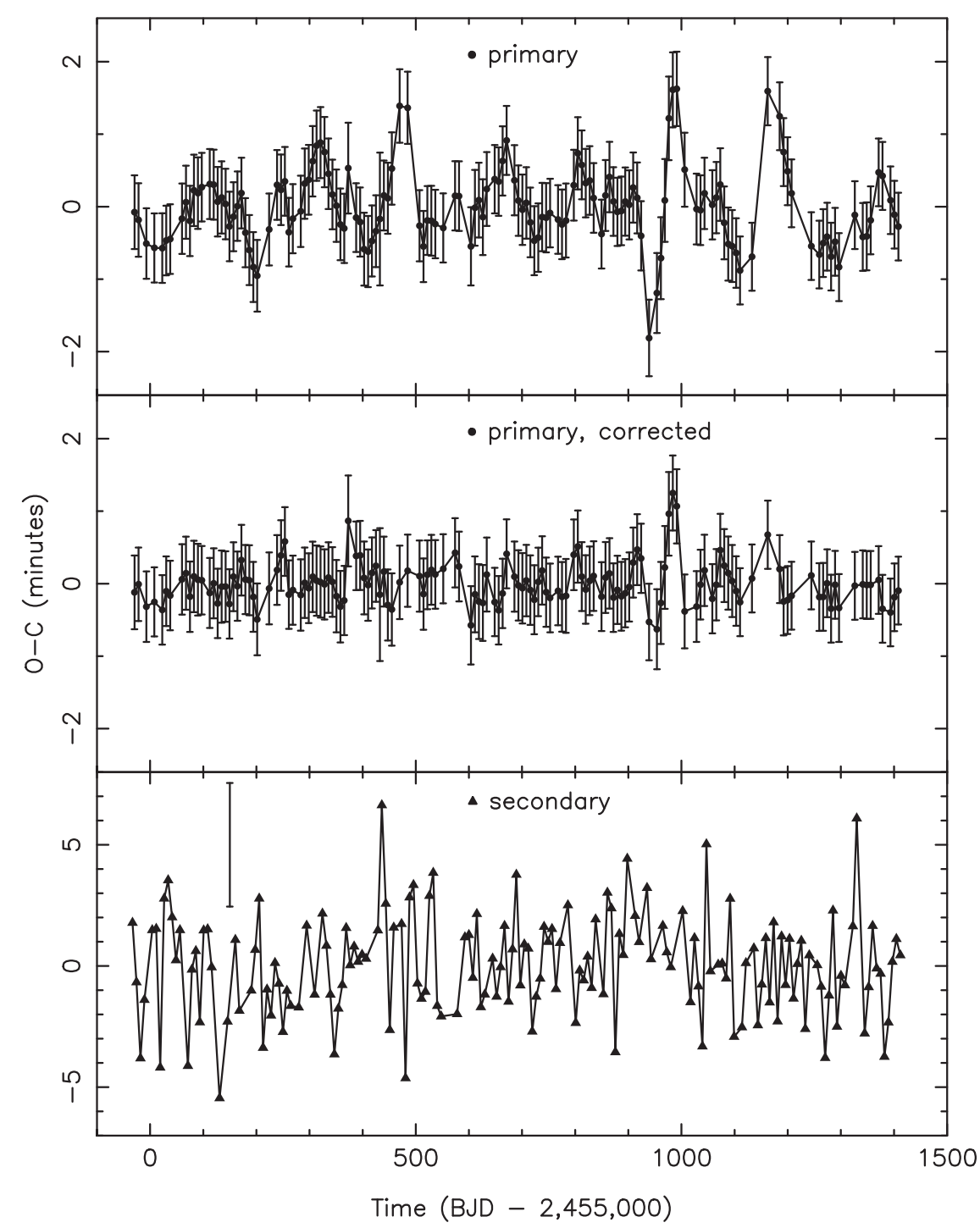

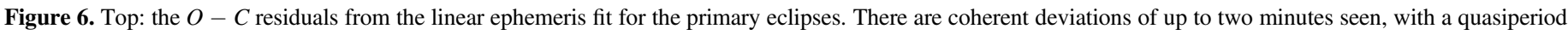

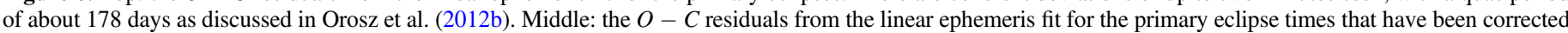

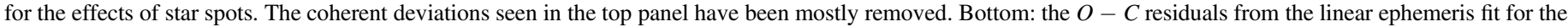

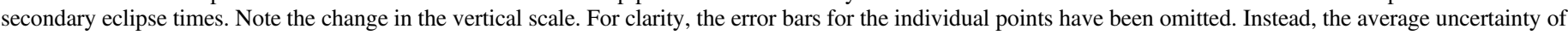
2.54 minutes is shown by the isolated error bar.

The integrator works in Cartesian coordinates relative to the system's barycenter. The unit of mass is the solar mass, the unit of distance is the astronomical unit, the unit of time is the day, and the unit of velocity is astronomical units per day. The adopted value of the Gaussian gravitational constant is $k=0.01720209895$ (Clemence 1965). Newton's gravitational constant, expressed in (au $)^{2} M_{\odot}{ }^{-1}(\text { day })^{-2}$, is then $G=k^{2}$. For convenience, instantaneous Keplerian parameters valid at some reference epoch for each body are used to specify the initial conditions (our adopted reference time is $T_{\text {ref }}=$ BJD $2,454,965.000)$. These parameters are the orbital period $P$, the time of barycentric transit (which is the same as the time of inferior conjunction) $T_{\text {conj }}$, the inclination $i$, the eccentricity $e$, the argument of periastron $\omega$, and the nodal angle $\Omega$. The coordinate system is Jacobian, so the orbital parameters of the secondary star are given relative to the primary, the orbital parameters of the first planet are given relative to the binary's center of mass, and so on. Because of this, the specified conjunction time for the binary occurs very near a primary eclipse, whereas nothing observable has to happen at the conjunction times of the planets (the actual transits can occur either earlier or later than the barycentric conjunction times). The Keplerian parameters are converted to Cartesian coordinates using the algorithms given in Murray \& Dermott (1999).

After the ODE is solved, the plane-of-sky positions of each body are corrected for light travel time following the method outlined in Carter et al. (2011). The times of the primary and secondary eclipses, and the times of transits of each planet across the primary and secondary are then computed by finding the times when the projected separation between two given bodies in the plane of the sky is minimized. For computational convenience, we minimize the square of the plane-of-sky distance vector, which is just the dot product of that distance vector with itself. That dot product is minimized when its derivative is zero. That derivative is two times the dot product of the distance vector with its velocity vector (which itself is readily found using the appropriate components of the velocities that are output from the ODE solver), and is zero 


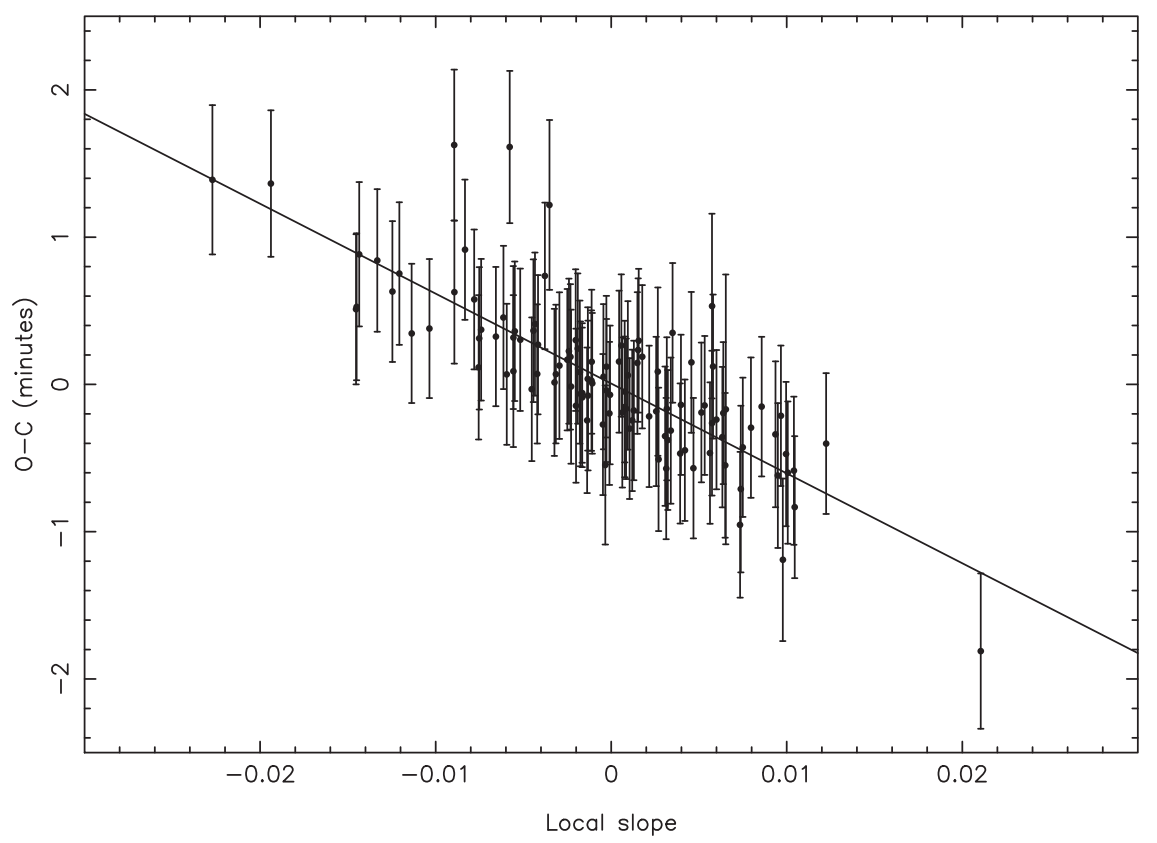

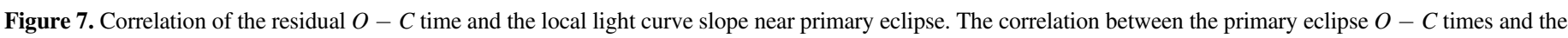
local slope in the SAP light curve is shown. The best-fitting line has a coefficient of correlation $r=-0.84$.

when the distance vector itself is zero or when the distance vector is perpendicular to its velocity vector. Several iterations of the secant method are used to find the zeros of the derivative. By comparing the results found using the first-order ODE solver with the results found using the second-order ODE solver, we estimate the times of conjunction to be accurate to $\approx 1 \mu \mathrm{s}$ or better. An eclipse or a transit will occur at a conjunction if the projected minimum separation on the sky is less than the sum of the radii of the two bodies.

For the specific case of Kepler-47, the corrections due to GR result in an apsidal advance of about 0.00017 per orbital period. The rate of apsidal advance due to tidal bulges on each star is between 10 and 50 times smaller, depending on the values of the tidal Love numbers used (we considered $0.0 \leqslant k_{2} \leqslant 0.01$ for the primary and $0.0 \leqslant k_{2} \leqslant 0.2$ for the secondary). In what follows, we have included the GR correction in the ODEs and neglected the corrections for the apsidal advance due to tidal bulges on the stars.

\subsection{Light Curve Synthesis}

For situations where the bodies are spherical with linear or quadratic limb darkening laws, ELC can use the algorithm of Mandel \& Agol (2002) or the algorithm of Giménez (2006a) to compute the light curves during eclipses or transits. In the present work, we have used the Mandel \& Agol (2002) routine with a quadratic limb darkening law, because it is much faster than the Giménez (2006a) routine.

\subsection{Model Setup for Kepler-47}

Our model as applied to Kepler-47 has 42 free parameters in total. Each orbit needs six Keplerian parameters for a total of 24. However, the nodal angle of the binary is fixed at zero, so there are really only 23 free parameters to describe the orbits. For each orbit, we used the combinations $e \cos \omega$ and $e \sin \omega$ rather than $e$ and $\omega$ separately, because the former pair of variables usually are less correlated with each other compared to the latter pair of variables. Because there are five bodies, five parameters describing the masses of the bodies and five parameters describing the sizes of the bodies are needed. For the binary, we used the primary mass $M_{1}$ and the binary mass ratio $Q_{2,1} \equiv M_{2} / M_{1}$. For the two stars, we used the fractional radii $R_{1} / a$ and $R_{2} / a$ to parameterize their sizes. To parameterize the planetary radii, we used the ratio $R_{1} / R_{p}$, which is the ratio of the primary star's radius to the particular planet radius in question. There are two radiating bodies in the system, so a total of six parameters are required to specify the radiative properties: the two temperatures and two limb darkening coefficients for each star. In this case, the temperature of the primary was fixed at $5636 \mathrm{~K}$ (Orosz et al. 2012b), so only five free parameters were used. For convenience, the temperature ratio $T_{2} / T_{1}$ was used instead of directly using $T_{2}$. ELC has a table of model atmosphere specific intensities (for solar metallicity) derived from the NextGen models (Allard et al. 1997; Hauschildt et al. 1999), and these were used to compute the baseline fluxes of the primary and secondary star (in the Kepler bandpass) given their temperatures and gravities. The standard quadratic limb darkening law given by

$$
I(\mu) / I_{0}=1-u_{1}(1-\mu)-u_{2}(1-\mu)^{2}
$$

was used (where $\mu=\cos \theta$ is the projected distance from the center of the stellar disk), but with the "triangular" sampling technique of Kipping (2013) with coefficients given by $q_{1}=\left(u_{1}+u_{2}\right)^{2}$ and $q_{2}=0.5 u_{1}\left(u_{1}+u_{2}\right)^{-1}$. Finally, to account for light from other sources in Kepler's aperture, four seasonal contamination parameters were used.

\subsection{Model Optimization}

As was shown in Section 4, many of the primary eclipse profiles show clear evidence of spot crossing events. Because our light curve model does not include star spots, fitting the primary eclipses where there are clear spot crossing events 
Table 4

Priors for Fitting Parameters

\begin{tabular}{|c|c|c|c|}
\hline Orbit & Parameter & Lower & Upper \\
\hline \multirow{15}{*}{$\frac{\text { Binary and }}{\underline{\text { Stellar }}}$} & Period (days) & 7.4480 & 7.44899 \\
\hline & & & \\
\hline & $T_{\text {conj }}($ BJD 2,455,000+) & -29.316 & -29.300 \\
\hline & $e \cos \omega$ & -0.1 & 0.0 \\
\hline & $e \sin \omega$ & -0.05 & 0.01 \\
\hline & Inclination (deg) & 89.00 & 90.25 \\
\hline & $M_{1}\left(M_{\odot}\right)$ & 0.80 & 1.0 \\
\hline & $Q$ & 0.340 & 0.373 \\
\hline & $R_{1} / a$ & 0.046 & 0.061 \\
\hline & $R_{2} / a$ & 0.0186 & 0.0202 \\
\hline & $T_{2} / T_{1}$ & 0.39 & 0.83 \\
\hline & $q_{1}$ (primary) & 0.0 & 1.0 \\
\hline & $q_{2}$ (primary) & 0.0 & 1.0 \\
\hline & $q_{1}($ secondary $)$ & 0.0 & 1.0 \\
\hline & $q_{2}$ (secondary) & 0.0 & 1.0 \\
\hline \multirow[t]{7}{*}{$\underline{\text { Inner }}$} & Period (days) & 49.41 & 49.52 \\
\hline & $T_{\text {conj }}($ BJD 2,455,000+) & -31.6 & -31.14 \\
\hline & $e \cos \omega$ & -0.08 & 0.10 \\
\hline & $e \sin \omega$ & 10.114 & 0.069 \\
\hline & Inclination (deg) & 89.03 & 90.32 \\
\hline & Nodal angle (deg) & -0.390 & 0.315 \\
\hline & $M_{3}\left(M_{\oplus}\right)$ & 0.01 & 100.0 \\
\hline \multirow[t]{7}{*}{ Middle } & Period (days) & 187.15 & 187.60 \\
\hline & $T_{\text {conj }}($ BJD 2,455,000+) & 45.10 & 45.75 \\
\hline & $e \cos \omega$ & -0.39 & 0.35 \\
\hline & $e \sin \omega$ & -0.1 & 0.2 \\
\hline & Inclination (deg) & 90.18 & 90.62 \\
\hline & Nodal angle (deg) & -2.8 & 0.5 \\
\hline & $M_{3}\left(M_{\oplus}\right)$ & 0.01 & 100.0 \\
\hline \multirow[t]{7}{*}{ Outer } & Period (days) & 303.00 & 303.42 \\
\hline & $T_{\text {conj }}($ BJD 2,455,000+) & -55.8 & -55.4 \\
\hline & $e \cos \omega$ & -0.19 & 0.23 \\
\hline & $e \sin \omega$ & -0.21 & 0.11 \\
\hline & Inclination (deg) & 90.10 & 90.28 \\
\hline & Nodal angle (deg) & -3.0 & 1.2 \\
\hline & $M_{3}\left(M_{\oplus}\right)$ & 0.01 & 100.0 \\
\hline \multirow[t]{4}{*}{ Kepler } & First season contamination $^{a}$ & 0.000 & 0.035 \\
\hline & $\begin{array}{l}\text { Second season } \\
\text { contamination }^{\mathrm{b}}\end{array}$ & 0.000 & 0.035 \\
\hline & Third season contamination ${ }^{c}$ & 0.000 & 0.035 \\
\hline & $\begin{array}{l}\text { Fourth season } \\
\text { contamination }^{\mathrm{d}}\end{array}$ & 0.000 & 0.035 \\
\hline
\end{tabular}

Notes.

a Kepler Quarters Q1, Q5, ..

${ }^{\mathrm{b}}$ Kepler Quarters Q2, Q6, ..

c Kepler Quarters Q3, Q7, ...

${ }^{\mathrm{d}}$ Kepler Quarters Q4, Q8, $\cdots$

might lead to biased results. To avoid this possible bias in the fitting, we fit the statistically corrected primary eclipse times along with only a small subset of the light curve. We chose ten primary eclipse profiles (seven in long cadence and three in short cadence) that do not have spot crossing events to fit, as well as ten secondary eclipses that are close in time to the ten selected primary eclipses. We also fit the 25 transits of the inner planet, the six transits of the middle planet, and the four transits of the outer planet. Finally, we also included the radial velocities of the primary star in the fit.

We used four algorithms to explore parameter space: an adaptation of a simple grid-search algorithm (Bevington 1969), a genetic algorithm (Charbonneau 1995); a simple search based the
MCMC algorithm outlined by Tegmark et al. (2004), and a Differential Evolution MCMC (DE-MCMC) algorithm of Ter Braak (2006; see also Nelson et al. 2014). All four of these algorithms are bounded, meaning the fitting parameters never wander outside predefined ranges. In addition, the grid-search code, the genetic code, and the DE-MCMC code can be run in parallel on multiple CPU cores. We assumed the likelihood function follows a $\chi^{2}$ distribution, where $\chi^{2}$ is based on the sum of squares of differences between observed quantities (a photometric measurement, a radial velocity measurement, or an eclipse or transit time) and predicted quantities normalized by the measurement uncertainty for each quantity. Each of these algorithms needs to have fixed ranges for each free parameter. Some parameters, like some of the orbital parameters for the binary, are constrained reasonably well. We also had some good starting solutions for the parameters of the inner planet from our previous work (Orosz et al. 2012b), whereas some of the orbital parameters (for example, the eccentricity parameters) for the outer planet were constrained much less well. Apart from the rough value of the orbital period and the fact that the inclination of the orbit must be near $90^{\circ}$ in order for transits to occur, the orbital parameters for the middle planet were initially not known.

Because the parameter space is vast, we looked for optimal solutions in three main stages: (i) updating the model from the previous work by adding the middle planet to the model and finding some initial fits; (ii) exploring parameter space to find something close to the "best" model, get some rough idea of the uncertainties in the fitted and derived parameters, and also look for possible correlations between various parameters; and (iii) finding realistic uncertainties on the fitted and derived parameters. The three stages are described below.

Stage $i$ : the inner planet has seven additional transits and the outer planet has one additional transit since the previous work, so the orbital parameters for those two planets were revised first using the DE-MCMC code. Next, the middle planet was added to the model, and initial orbital parameters were found "by hand." Educated guesses for the time of barycentric conjunction, the inclination, the nodal angle, etc. were used to initialize the simple MCMC code, and the model fits were inspected by eye after running a few iterations of that optimizer. It was not unduly difficult to find a model that produced transits of the middle planet at roughly the correct times.

Stage ii: after a few decent models were found, longer runs using the genetic algorithm and the DE-MCMC were done. Both of these codes require an initial "population" of models (we typically used population sizes between 100 and 200), and we have various ways of generating the initial population: (a) totally random values between the specified lower and upper parameter bounds for each free parameter; (b) one or more "elite" models (e.g., ones that are already a good match to the data) along with randomly generated models; and (c) one or more "elite" models along with "mutated" copies of the elite models where a few randomly chosen parameters are "tweaked" by adding or subtracting small offsets. In practice, options (b) and (c) work the best for cases where one has a few dozen or more free parameters.

For this intermediate stage of fitting, we adopted ranges for the free parameters given in Table 4. We ran the genetic algorithm or the DE-MCMC code for a few pilot runs of several thousand generations to confirm that the prior ranges included support for the entire range with nontrivial likelihood. After each pilot run, plots of parameter values versus the 

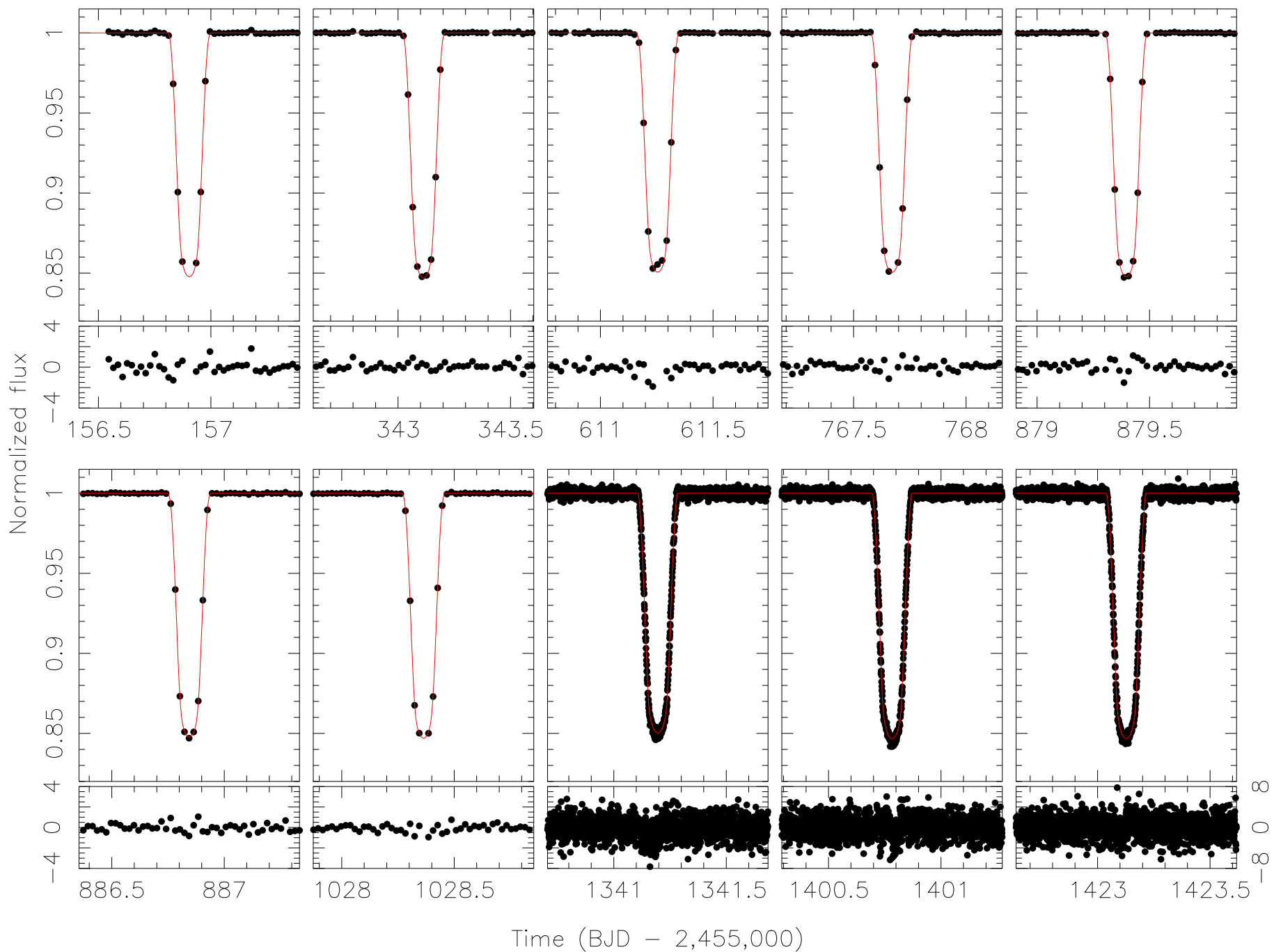

Figure 8. Model fits to the "clean" primary eclipse profiles. The fits to the 10 primary eclipse profiles that have no strong spot crossing events are shown. The vertical scale on the panels showing the residuals are parts per thousand. Note the scale change on the three panels showing the residuals of the fits to the short-cadence data.

generation number and plots of the $\chi^{2}$ versus each parameter were inspected to find instances where the allowed range of a parameter was either too large or too small. We verified that the likelihood falls to extremely small values by the time model parameters reach any of these boundaries (with the possible exception of hard physical boundaries).

After this intermediate state of optimization, several million models have been computed. The genetic algorithm and the DE-MCMC code explore parameter space in very different ways; after several long runs of each, we can be reasonably sure the optimal region in parameter space has been found.

Stage iii: We used a brute-force "stepper" to generate initial "seed" models for the final run of the DE-MCMC code. The stepper works as follows. Take the best overall model and choose a key fitting parameter, such as the primary mass. Offset that parameter by a small amount (say, $0.5 \%$ of the parameter value) and hold it fixed while optimizing the other parameters using the simple parallel grid-search code, where the parameter values from the optimal model are used as the initial guess. After that optimization is done, offset the same key parameter again and repeat the optimization, using the previous solution as the initial guess. After the stepper is done, you have a set of reasonably optimal models with a range of different values of the chosen key parameter. We ran steppers on the mass parameters (primary mass, binary mass ratio, and the planet masses), the radius parameters (primary and secondary fractional radii and planet radius ratios), and the planet orbital periods.

For the last run of the DE-MCMC code, we used a population size of 1600 models per generation with 900 "seed" models found from the steppers. The other 700 models were mutated copies of randomly selected seed models with five parameters in each model randomly tweaked. The code was ran for 17,000 generations, giving about 27 million models in total. Judging from plots of the parameters versus the generation number, convergence was achieved after about 4000 generations. However, to be conservative, the first 5000 generations were discarded, leaving about 19 million models. Figure 1 shows the best-fitting model radial velocity curve. The best fits to the transits of the middle planet, inner planet, and the outer planet are shown in Figures 2-4, respectively. Figure 8 shows the best-fitting model with the "clean" primary eclipses and Figure 9 shows the best-fitting model with the corresponding secondary eclipses. The best-fitting input parameters are given 


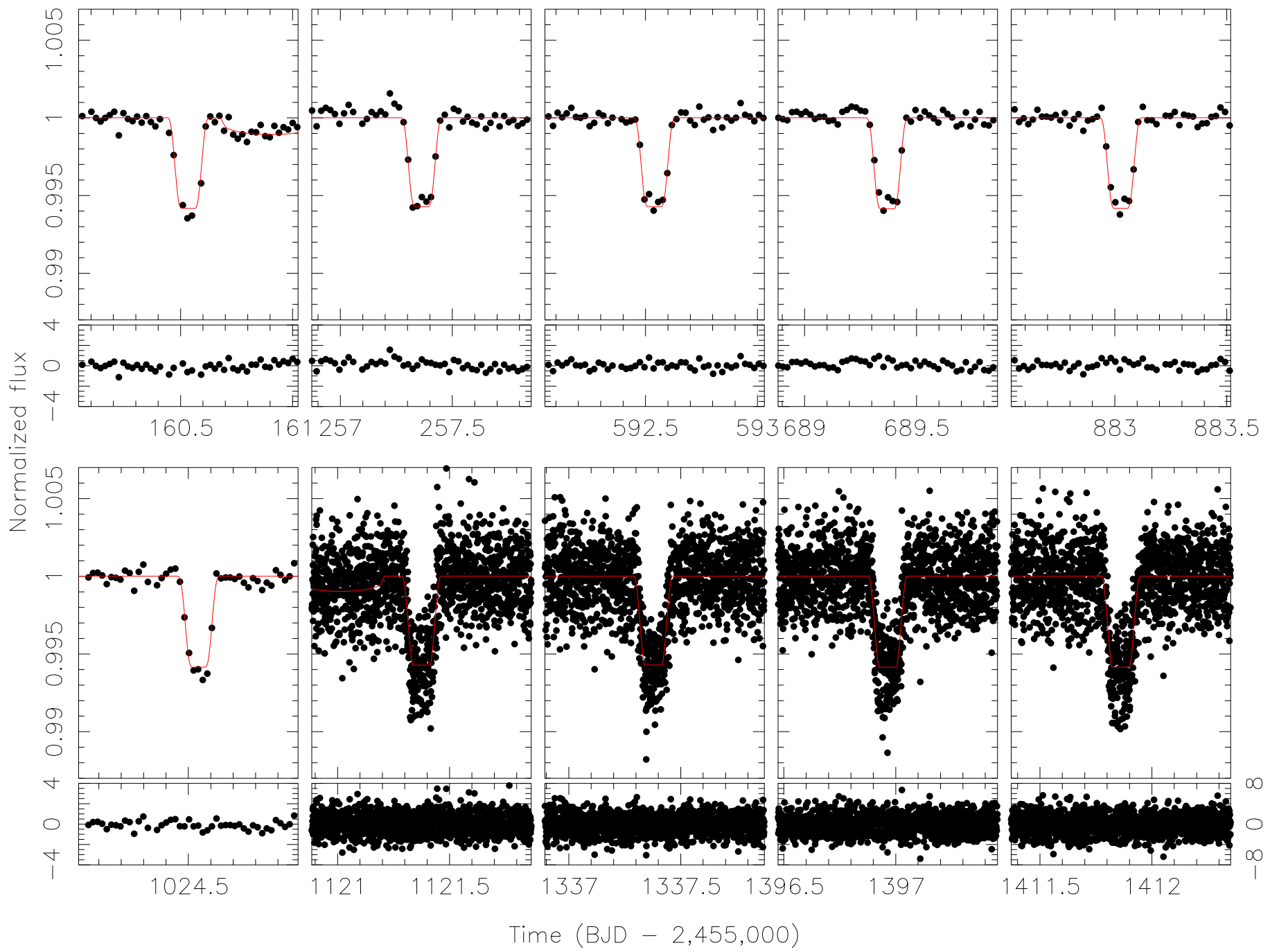

Figure 9. Model fits to the "clean" secondary eclipse profiles. The fits to the 10 secondary eclipse profiles included in the fitting are shown. The vertical scale on the panels showing the residuals are parts per thousand. Note the scale change on the four panels showing the residuals of the fits to the short-cadence data. Transits of the inner planet can be seen near day 160.7 and day 1121.0.

in Table 5, and Table 6 summarizes several derived parameters of interest. In order to allow others to reproduce our best-fitting model using other codes, we give the initial Cartesian barycentric coordinates for the best-fitting model in Table 7 and the initial orbital elements (which are traditionally used in dynamical studies) for the best-fitting model in Table 8 . These coordinates and orbital elements are valid for the reference time BJD 2,454,965.000.

For each parameter's posterior distribution, we computed the mode and the lower and upper boundaries of the region that contains $68.3 \%$ of the area under the curve. We show plots of posterior distributions for several fitting and derived parameters in Figures 10-17, and plots of the two-parameter joint posterior distributions for the 28 major orbital parameters in Figures 18-20 (Table 9 gives the displayed parameters and their ranges).

When one examines the posterior distribution of a given parameter, the mode of the distribution might be taken as the "most likely" value of that parameter. We note, however, that a model constructed using the modes of each distribution will not necessarily provide a good fit to the data. Therefore, for the final adopted parameters, we take the parameters from the bestfitting model.

\section{Discussion}

\subsection{Constraints on the Planetary Masses}

For several CBPs (Doyle et al. 2011; Welsh et al. 2012; Kostov et al. 2016), the gravitational pull of the planet is sufficiently large to measurably alter the positions and velocities of their host stars. These perturbations can be detected by measuring slight deviations from strict periodicity in the times of the stellar eclipses. From these eclipse-timing variations, the mass of the planet can be estimated. For Kepler47 , only upper limits on the masses could be made at the time of their discovery: $<2.0$ Jupiter masses $\left(<635 M_{\oplus}\right)$ for the inner planet and $<28$ Jupiter masses $\left(<8900 M_{\oplus}\right)$ for the outer planet (Orosz et al. 2012b).

The presence of the third planet dramatically changes this situation. The two outer planets gravitationally perturb one another, resulting in dynamical transit-timing variations. Due to their proximity, the planets perturb each other more than they affect the stars. In the discovery paper (Orosz et al. 2012b), the planets were assumed to be massless in the modeling process, as the interactions between the planets and the binary-and between each other-was determined to be small. In the present model, all five bodies have mass and the mutual 
Table 5

Fitted Parameters from the Photometric-dynamical Model ${ }^{\mathrm{a}}$

\begin{tabular}{|c|c|c|c|c|c|}
\hline Parameter & Binary Orbit & Inner Orbit & Middle Orbit & Outer Orbit & \\
\hline Period (days) & $7.4483648_{-0.0000270}^{+0.0000038}$ & $49.4643_{-0.0074}^{+0.0081}$ & $187.366_{0.051}^{+0.069}$ & $303.227_{-0.027}^{+0.062}$ & \\
\hline$T_{\text {conj }}(\mathrm{BJD}+2,455,000)$ & $-29.305248_{-0.000030}^{+0.000031}$ & $-31.3573_{-0.0049}^{+0.0043}$ & $45.428_{-0.047}^{+0.044}$ & $-55.638_{-0.046}^{+0.067}$ & \\
\hline$e \cos \omega$ & $-0.019895_{-0.000024}^{+0.000025}$ & $0.0139_{-0.0025}^{+0.0020}$ & $0.024_{-0.036}^{+0.038}$ & $0.026_{-0.034}^{+0.029}$ & \\
\hline$e \sin \omega$ & $-0.0208_{-0.0020}^{+0.0019}$ & $0.0157_{-0.0011}^{+0.0015}$ & $-0.004_{-0.028}^{+0.023}$ & $-0.035_{-0.025}^{+0.026}$ & \\
\hline$i(\operatorname{deg})$ & $89.613_{-0.040}^{+0.045}$ & $89.752_{-0.045}^{+0.063}$ & $90.395_{-0.012}^{+0.009}$ & $90.1925_{-0.0042}^{+0.0055}$ & \\
\hline$\Omega(\operatorname{deg})$ & 0.0 & $-0.088_{-0.053}^{+0.056}$ & $-0.87_{-0.10}^{+0.11}$ & $-1.28_{-0.25}^{+0.23}$ & \\
\hline Parameter & Primary & Secondary & Inner planet & Middle planet & Outer planet \\
\hline$R / a^{\mathrm{b}}$ & $0.05347_{-0.00016}^{+0.00016}$ & $0.019332_{-0.000065}^{+0.000056}$ & $\ldots$ & $\ldots$ & $\ldots$ \\
\hline$R_{1} / R^{\mathrm{c}}$ & $\cdots$ & $\ldots$ & $33.51_{-0.48}^{+0.54}$ & $14.55_{-1.35}^{+1.11}$ & $21.97_{-0.35}^{+0.42}$ \\
\hline$q_{1}^{\mathrm{d}}$ & $0.502_{-0.063}^{+0.067}$ & $<0.66$ & $\ldots$ & $\ldots$ & $\ldots$ \\
\hline$q_{2}{ }^{\mathrm{e}}$ & $0.188_{-0.028}^{+0.020}$ & $0.38_{-0.12}^{+0.34}$ & $\cdots$ & $\cdots$ & $\cdots$ \\
\hline \multicolumn{6}{|l|}{ Other parameters } \\
\hline$s_{3}$ (Season 3 contamination) & $0.0232_{-0.0029}^{+0.0007}$ & $\cdots$ & $\cdots$ & $\ldots$ & $\cdots$ \\
\hline
\end{tabular}

Notes.

${ }^{\mathrm{a}} T_{\text {ref }}=$ BJD 2,454,965.000.

${ }^{\mathrm{b}}$ Fractional stellar radii.

${ }^{\mathrm{c}}$ Ratio of primary radius to planet radius.

${ }^{\mathrm{d}}$ Kipping's first quad-law triangular limb darkening coefficient.

e Kipping's second quad-law triangular limb darkening coefficient.

Table 6

Derived Parameters from the Photometric-dynamical Model

\begin{tabular}{|c|c|c|c|c|c|}
\hline Parameter & Binary Orbit & Inner Orbit & Middle Orbit & Outer Orbit & \\
\hline$\overline{a(\mathrm{au})}$ & $0.08145_{-0.00037}^{+0.00036}$ & $0.2877_{-0.0011}^{+0.0014}$ & $0.6992_{-0.0033}^{+0.0031}$ & $0.9638_{-0.0044}^{+0.0041}$ & \\
\hline$e$ & $0.0288_{-0.0013}^{+0.0015}$ & $0.0210_{-0.0022}^{+0.0025}$ & $0.024_{-0.017}^{+0.025}$ & $0.044_{-0.019}^{+0.029}$ & \\
\hline$\omega(\operatorname{deg})$ & $226.3_{-2.6}^{+2.8}$ & $48.6_{-2.3}^{+3.0}$ & $352_{-166}^{+96}$ & $306_{-67}^{+40}$ & \\
\hline$I_{2, i}(\mathrm{deg})^{\mathrm{b}}$ & $\ldots$ & $\ldots$ & $1.006_{-0.050}^{+0.089}$ & $1.247_{-0.018}^{+0.024}$ & \\
\hline$I_{3, i}(\mathrm{deg})^{\mathrm{c}}$ & $\ldots$ & $\ldots$ & ... & $0.442_{-0.102}^{+0.028}$ & \\
\hline$R\left(R_{\odot}\right.$ or $\left.R_{\oplus}\right)$ & $0.936_{-0.005}^{+0.005}$ & $0.338_{-0.002}^{+0.0002}$ & $3.05_{-0.04}^{+0.04}$ & $7.04_{-0.49}^{+0.66}$ & $4.65_{-0.07}^{+0.09}$ \\
\hline$\rho\left(\mathrm{g} \mathrm{cm}^{-3}\right)$ & $1.65_{-0.01}^{+0.02}$ & $12.42_{-0.11}^{+0.11}$ & $0.40_{-0.40}^{+4.87}$ & $0.30_{-0.18}^{+0.38}$ & $0.17_{-0.07}^{+0.09}$ \\
\hline
\end{tabular}

Notes.

${ }^{a}$ Mutual inclination between the orbital planes of the binary and the planets, given by $\cos I=\sin i_{p} \sin i_{b} \cos \Delta \Omega+\cos i_{p} \cos i_{b}$.

${ }^{\mathrm{b}}$ Mutual inclination between the orbital planes of the inner planet and the other planets.

${ }^{c}$ Mutual inclination between the orbital planes of the middle planet and the outer planet.

interactions between each body are fully accounted for. We find that we can place meaningful constraints on the masses of the middle and outer planets. The $1 \sigma$ range of the mass of the inner planet is between zero and $25.8 M_{\oplus}$, whereas the mass of the middle planet is different from zero at the $\approx 5 \sigma$ limit and the mass of the outer planet is different from zero at the $\approx 2 \sigma$ limit.

We computed the planet transit times of the best model with a zero-mass middle planet and the best model with a zero-mass outer planet, and compared those times to the transit times from the overall best-fitting model. The difference in the respective transit times are shown in Figure 21. In either case, the transit times of the inner planet do not change by more than $\approx 1$ minute. On the other hand, a massless middle planet results in changes in the outer planet's transit times by up to $\approx 20$ minutes. Likewise, a massless outer planet results in changes in the middle planet's transit times by up to $\approx 10$ minutes. These changes in the transit times can be detected at the significance of a few $\sigma$, leading to the mass constraints that we have on the middle and outer planets.

Uncertainties in the planet masses have improved by over an order of magnitude compared with the discovery paper (Orosz et al. 2012b). The mass estimates for the inner and outer planets 
Table 7

Initial Cartesian Barycentric Conditions ${ }^{\mathrm{a}}$ for Kepler-47

\begin{tabular}{|c|c|c|c|c|c|}
\hline Parameter & Primary & Secondary & Inner Planet & Middle Planet & Outer Planet \\
\hline$\overline{\text { Mass }\left(M_{\odot}\right)}$ & $9.573912601143495 \mathrm{E}-01$ & $3.416626576742950 \mathrm{E}-01$ & $6.208164703811071 \mathrm{E}-06$ & $5.711706000000001 \mathrm{E}-05$ & $9.530601983442541 \mathrm{E}-06$ \\
\hline$x(\mathrm{au})$ & $2.092317942847221 \mathrm{E}-02$ & $-5.866162141774457 \mathrm{E}-02$ & $1.302102743834203 \mathrm{E}-01$ & $2.398289447308760 \mathrm{E}-01$ & $-3.872087634688049 \mathrm{E}-01$ \\
\hline$y(\mathrm{au})$ & $-1.364205200783173 \mathrm{E}-05$ & $3.791628623967441 \mathrm{E}-05$ & $8.812713793817102 \mathrm{E}-04$ & $8.391663271667140 \mathrm{E}-04$ & $5.540095504781927 \mathrm{E}-03$ \\
\hline$z(\mathrm{au})$ & $-1.988193746560007 \mathrm{E}-03$ & $5.649120240556342 \mathrm{E}-03$ & $2.500211844794519 \mathrm{E}-01$ & $-6.478290001132635 \mathrm{E}-01$ & $9.270974333301998 \mathrm{E}-01$ \\
\hline$v_{x}(\mathrm{au})$ & $1.350420386068420 \mathrm{E}-03$ & $-3.786681230811973 \mathrm{E}-03$ & $-3.299902928667536 \mathrm{E}-02$ & $2.207346827176963 \mathrm{E}-02$ & $-1.773342551338714 \mathrm{E}-02$ \\
\hline$v_{y}(\mathrm{au})$ & $1.239137665559171 \mathrm{E}-04$ & $-3.471736278879929 \mathrm{E}-04$ & $1.256761297656883 \mathrm{E}-04$ & $-3.936013341278077 \mathrm{E}-04$ & $4.205823582940745 \mathrm{E}-04$ \\
\hline$v_{z}(\mathrm{au})$ & $1.835286133723581 \mathrm{E}-02$ & $-5.142910738078619 \mathrm{E}-02$ & $1.739447523236208 \mathrm{E}-02$ & $8.701783937203954 \mathrm{E}-03$ & $-7.191666020340975 \mathrm{E}-03$ \\
\hline
\end{tabular}

Note.

${ }^{\mathrm{a}} T_{\text {ref }}=$ BJD 2,454,965.000. 
Table 8

Initial Orbital Elements ${ }^{\mathrm{a}}$ for Kepler-47

\begin{tabular}{|c|c|c|c|c|}
\hline Parameter & $\begin{array}{c}\text { Binary } \\
\text { Orbit }\end{array}$ & $\begin{array}{l}\text { Inner } \\
\text { Orbit }\end{array}$ & $\begin{array}{c}\text { Middle } \\
\text { Orbit }\end{array}$ & $\begin{array}{l}\text { Outer } \\
\text { Orbit }\end{array}$ \\
\hline$a(\mathrm{au})$ & $8.144243202775878 \mathrm{E}-02$ & $2.877434527504005 \mathrm{E}-01$ & $6.992154891162726 \mathrm{E}-01$ & $9.638218771090775 \mathrm{E}-01$ \\
\hline$e$ & $2.877193854136767 \mathrm{E}-02$ & $2.095514505946130 \mathrm{E}-02$ & $2.416231575331903 \mathrm{E}-02$ & $4.371716413425727 \mathrm{E}-02$ \\
\hline$i(\mathrm{deg})$ & $8.961321083430335 \mathrm{E}+01$ & $8.975235828571519 \mathrm{E}+01$ & $9.039477337702283 \mathrm{E}+01$ & $9.019249877520613 \mathrm{E}+01$ \\
\hline$\omega(\operatorname{deg})$ & $2.262537367634179 \mathrm{E}+02$ & $4.859496576626187 \mathrm{E}+01$ & $3.517243342867096 \mathrm{E}+02$ & $3.059357996708584 \mathrm{E}+02$ \\
\hline$\Omega(\operatorname{deg})$ & $0.000000000000000 \mathrm{E}+00$ & $-8.764673739309498 \mathrm{E}-02$ & $-8.659498733670760 \mathrm{E}-01$ & $-1.280580223423379 \mathrm{E}+00$ \\
\hline$f(\mathrm{deg})$ & $3.082645652441405 E+02$ & $1.389173589110049 \mathrm{E}+01$ & $2.985868391504580 \mathrm{E}+02$ & $1.667337092860433 \mathrm{E}+02$ \\
\hline$M(\mathrm{deg})$ & $3.108188471954695 \mathrm{E}+02$ & $1.332389759998393 \mathrm{E}+01$ & $3.009969996738955 \mathrm{E}+02$ & $1.655463689900479 \mathrm{E}+02$ \\
\hline$\lambda(\operatorname{deg})$ & $1.745183020075583 \mathrm{E}+02$ & $6.239905491996927 \mathrm{E}+01$ & $2.894452235638005 \mathrm{E}+02$ & $1.113889287334783 \mathrm{E}+02$ \\
\hline
\end{tabular}

Notes.

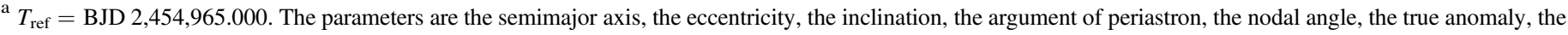
mean anomaly, and the mean longitude.

Table 9

Parameter Ranges Displayed in Figures 18-20

\begin{tabular}{|c|c|c|c|}
\hline Orbit & Parameter & Lower & Upper \\
\hline \multirow[t]{7}{*}{ Binary } & Period (days) & 7.44820 & 7.44838 \\
\hline & $T_{\text {conj }}(\mathrm{BJD} 2,455,000+)$ & -29.30541 & -29.30515 \\
\hline & $e \cos \omega$ & -0.02000 & -0.01979 \\
\hline & $e \sin \omega$ & -0.028 & -0.012 \\
\hline & Inclination (deg) & 89.5 & 89.8 \\
\hline & $M_{1}\left(M_{\odot}\right)$ & 0.8950 & 0.9905 \\
\hline & $M_{2}\left(M_{\odot}\right)$ & 0.326 & 0.352 \\
\hline \multirow[t]{7}{*}{$\underline{\text { Inner }}$} & Period (days) & 49.440 & 49.495 \\
\hline & $T_{\text {conj }}(\mathrm{BJD} 2,455,000+)$ & -31.375 & -31.337 \\
\hline & $e \cos \omega$ & 0.006 & 0.024 \\
\hline & $e \sin \omega$ & 0.0122 & 0.0210 \\
\hline & Inclination (deg) & 89.55 & 90.05 \\
\hline & Nodal angle (deg) & -0.32 & 0.12 \\
\hline & $M_{3}\left(M_{\oplus}\right)$ & 0.0 & 100.0 \\
\hline \multirow[t]{7}{*}{$\underline{\text { Middle }}$} & Period (days) & 187.2 & 187.5 \\
\hline & $T_{\text {conj }}(\mathrm{BJD} 2,455,000+)$ & 45.25 & 45.60 \\
\hline & $e \cos \omega$ & -0.15 & 0.14 \\
\hline & $e \sin \omega$ & -0.08 & 0.09 \\
\hline & Inclination (deg) & 90.34 & 90.45 \\
\hline & Nodal angle (deg) & -0.4 & -0.4 \\
\hline & $M_{3}\left(M_{\oplus}\right)$ & 0.0 & 100.0 \\
\hline \multirow[t]{7}{*}{$\underline{\text { Outer }}$} & Period (days) & 303.2 & 303.37 \\
\hline & $T_{\text {conj }}(\mathrm{BJD} 2,455,000+)$ & -55.78 & -55.49 \\
\hline & $e \cos \omega$ & -0.10 & 0.13 \\
\hline & $e \sin \omega$ & -0.110 & 0.055 \\
\hline & Inclination (deg) & 90.17 & 90.21 \\
\hline & Nodal angle (deg) & -2.4 & -0.4 \\
\hline & $M_{3}\left(M_{\oplus}\right)$ & 0.0 & 12.0 \\
\hline
\end{tabular}

are now $<26 M_{\oplus}$ and $\sim 2-5 M_{\oplus}$, at the $1 \sigma$ level. The middle planet has a mass $\sim 7-43 M_{\oplus}(1 \sigma$ range $)$. As usual, the radii are determined much better than the masses: $3.05,7.0$, and $4.7 R_{\oplus}$ for the inner, middle, and outer planets, respectively. The middle and outer planets have low bulk densities, $<0.68$ and $<0.26 \mathrm{~g} \mathrm{~cm}^{-3}$ at the $1 \sigma$ level (Saturn's density is $0.69 \mathrm{~g} \mathrm{~cm}^{-3}$ ). The densities of all currently known low-mass planets are shown in Figure 22; models indicate that such low-density planets must have substantial hydrogen and helium atmospheres (Lopez \& Fortney 2014; Jontof-Hutter et al. 2016). There is also an apparent trend that highly irradiated planets tend to have high densities while planets with low incident fluxes have a range of densities. Kepler-47 c and Kepler-47 d fit this latter trend.

Several studies have shown that the formation of CBPs at close distances to the binary is not expected to proceed efficiently, and that CBPs likely formed at large distances and migrated to their current orbits (Pierens \& Nelson 2008, 2013; Meschiari 2012; Paardekooper et al. 2012; Pelupessy \& Portegies Zwart 2013; Martin et al. 2013; Rafikov 2013; Kley \& Haghighipour 2014, 2015; Lines et al. 2015b). Planets that survive the migration phase are expected to have orbits with small eccentricities and small mutual inclinations relative to the orbital plane of the binary stars. The planets in Kepler- 47 have low masses compared with Jupiter or Saturn, and the orbits have low eccentricities: $e<0.030,0.024,0.05,0.07$ (at the $+1 \sigma$ level) for the binary and the inner, middle, and outer planets, respectively. All four orbits have mutual inclinations aligned to within 1.6 of one another $(+1 \sigma$ level). This nearly circular, co-planar, packed configuration is unlikely to have arisen as an outcome of strong gravitational scattering of the planets into their current orbits. Rather, the observations suggest that this planetary configuration is the result of relatively gentle migration in a circumbinary protoplanetary disk.

\subsection{Stability of the Orbits}

Numerical integrations have shown that the region around the binary where the orbit of a planet will become unstable extends to $\approx 0.18$ au (Hinse et al. 2015). Thus, the semimajor axis of the innermost planet $(0.2877 \mathrm{au})$ is much farther beyond the outer edge of the unstable region. The integrations of the observed system (using the nominal masses given in Table 6) also show evidence of stability, as the variations in the semimajor axis, eccentricity, ascending node, and inclination relative to the binary plane, $i_{\text {rel }}$, do not have appreciable secular changes and are remarkably flat in their maximum and minimum values over the $100 \mathrm{Myr}$ integration timescale (see Figure 23). The angular momentum deficit (AMD) of the system is used to estimate the changes of secular perturbations that could induce instability on a long timescale. We scale this quantity relative to the present-day solar system value for the terrestrial planets. The Kepler-47 planets are more massive than our terrestrial system, thereby allowing for higher values in AMD. Finally, we show a periodogram for the cyclical variations in inclination within the system and find the 

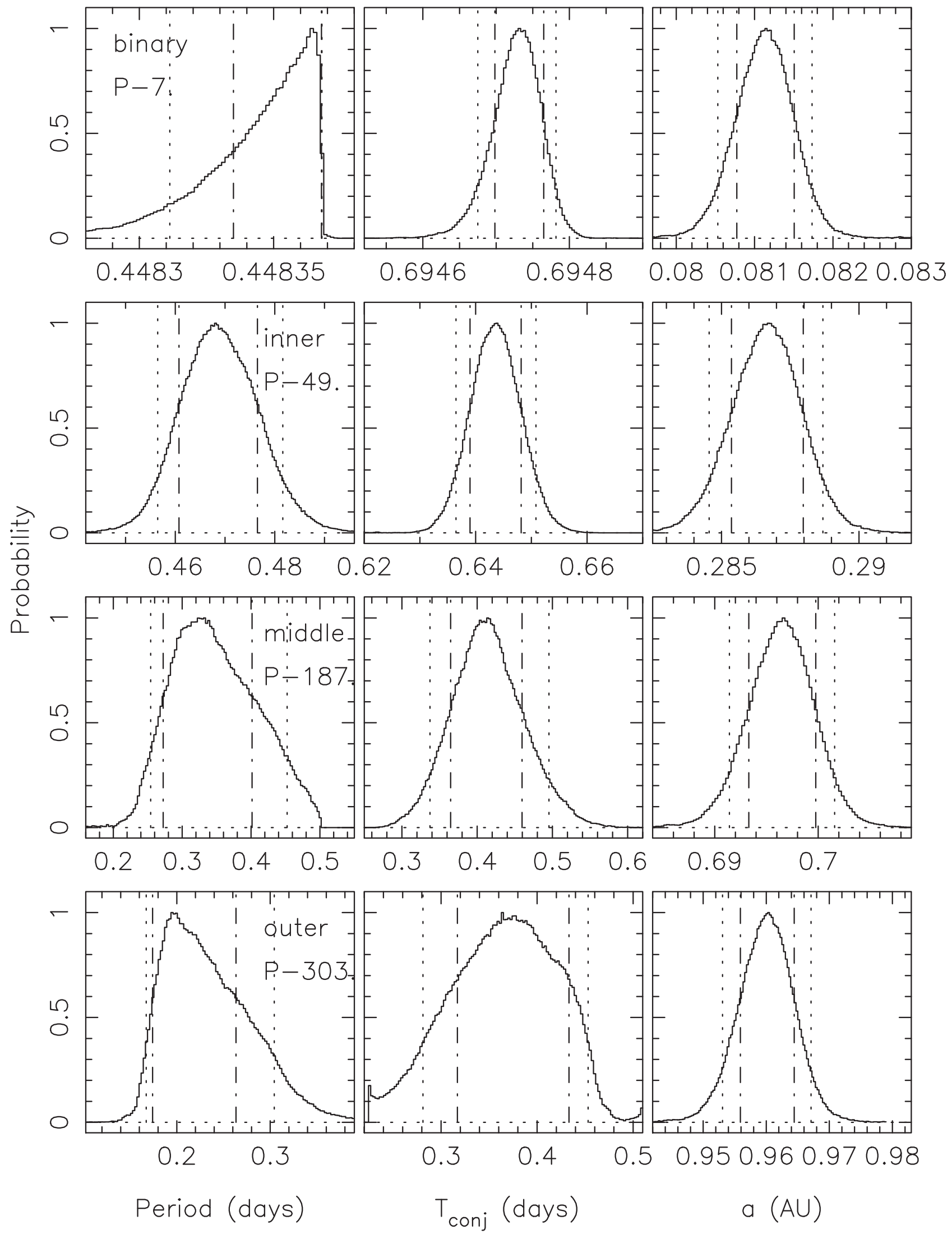

Figure 10. Posterior distributions from the DE-MCMC run of the orbital periods (left), times of barycentric conjunction (middle), and semimajor axes (right). We show only the decimal parts of the periods and times of conjunction (see Table 5 for parameter values from the best-fitting model). The dashed vertical lines denote the lower and upper boundaries that contain $68.3 \%$ of the area, and the vertical dotted lines denote the lower and upper boundaries that contain $90 \%$ of the area. 

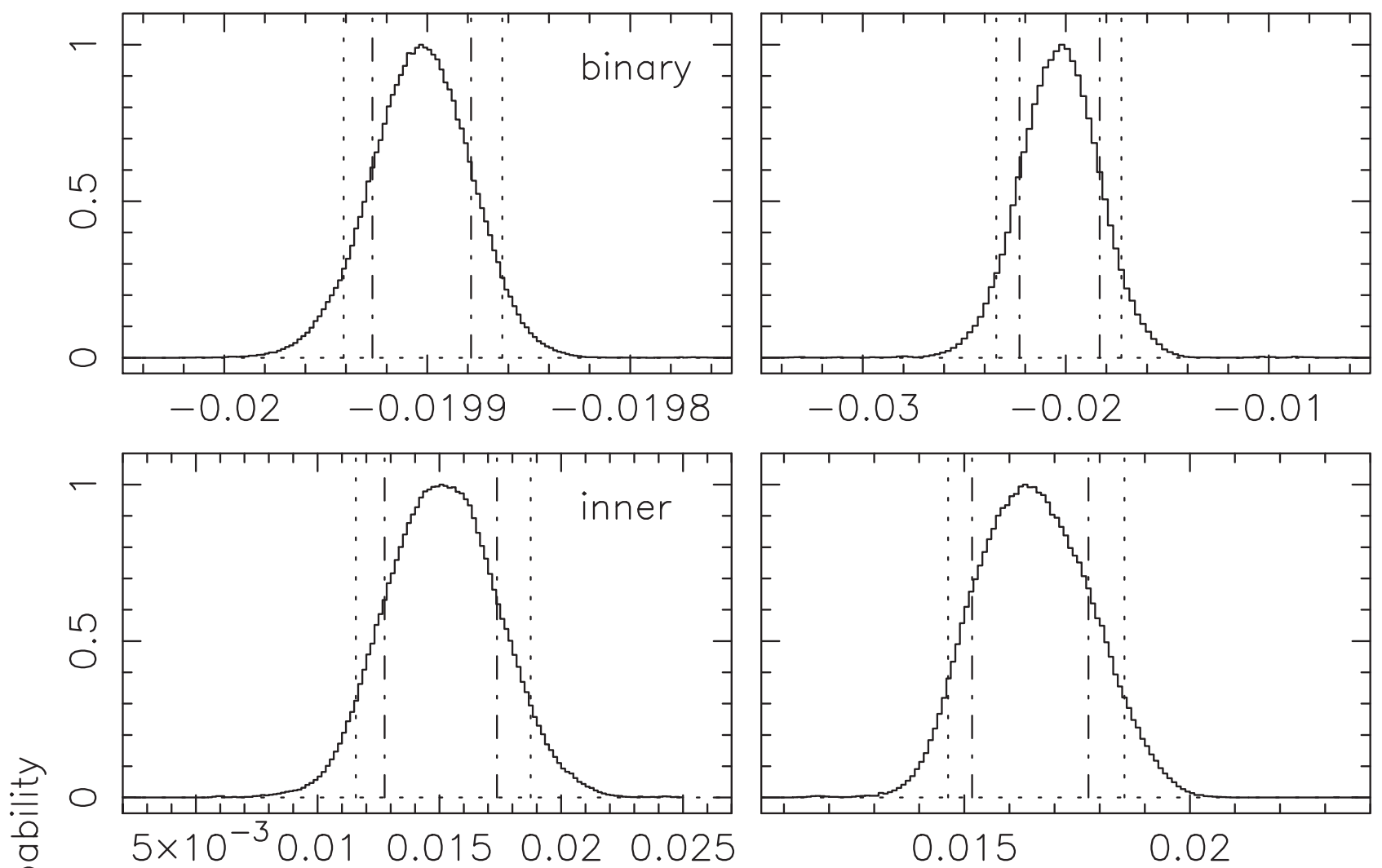

은
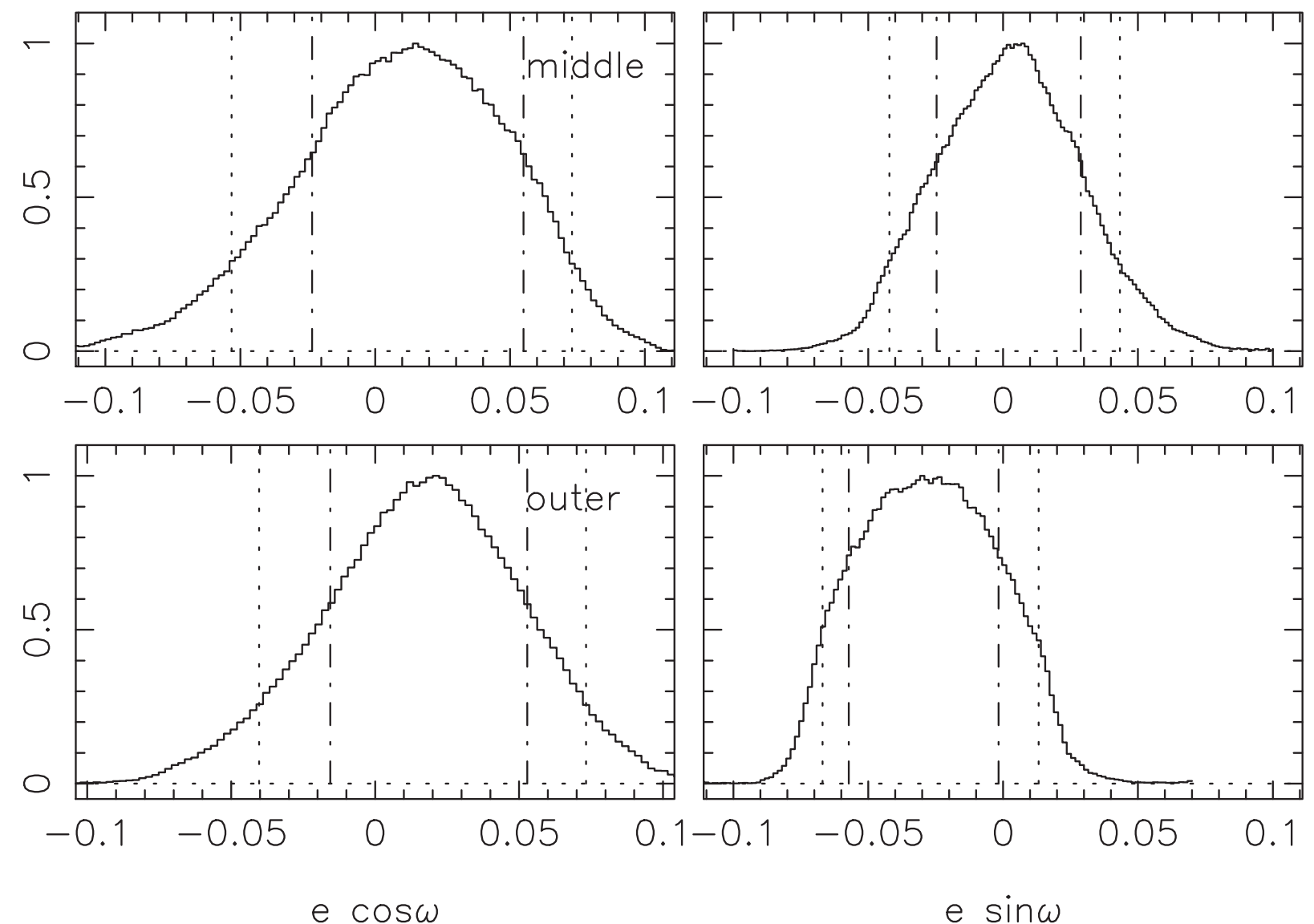

Figure 11. Similar to Figure 10, but showing the posterior distributions of $e \cos \omega$ (left) and $e \sin \omega$ (right). 

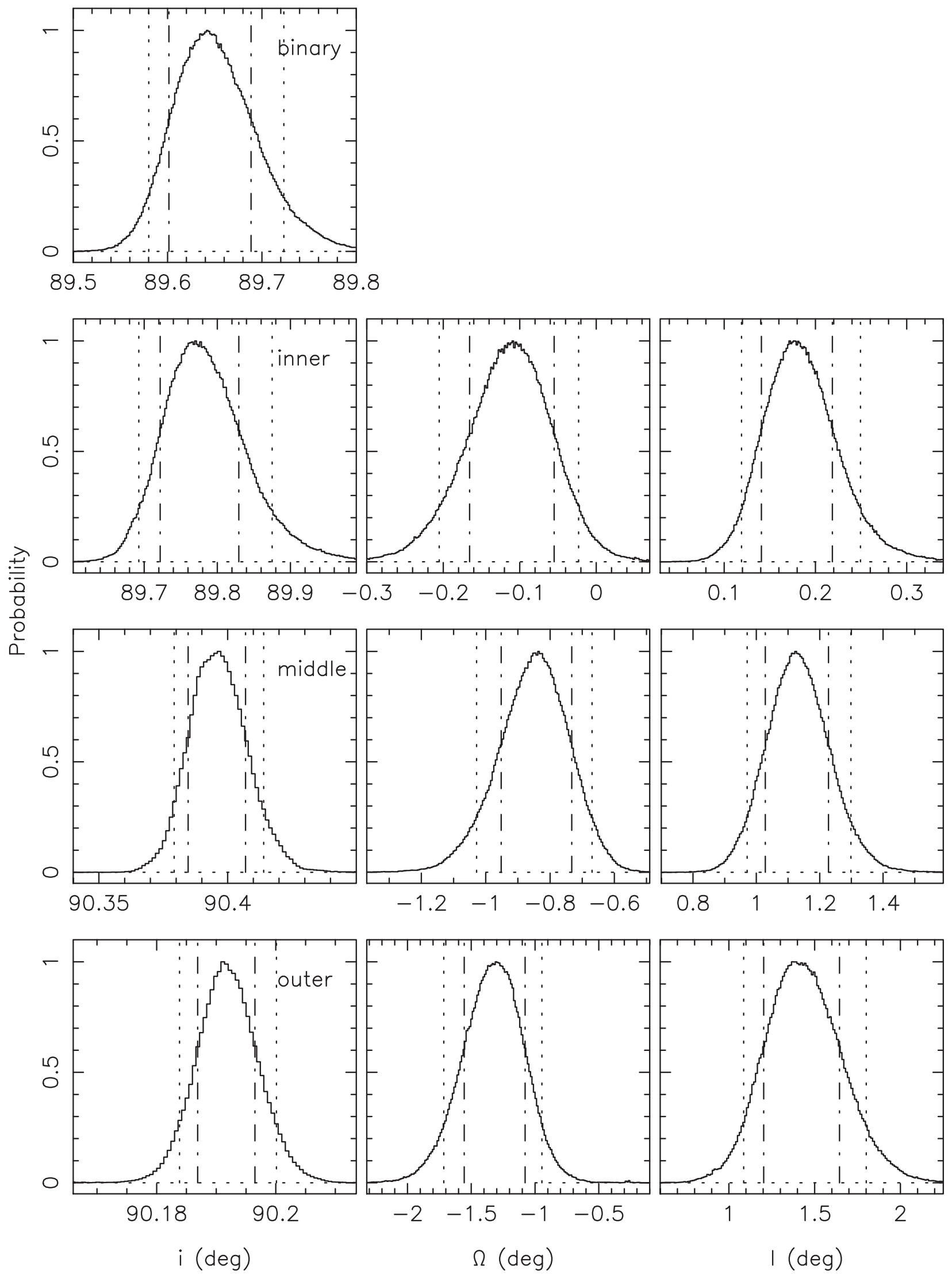

Figure 12. Similar to Figure 10, but showing the posterior distributions of the orbital inclinations (left); the nodal angles of the orbits-note that the nodal angle of the binary is fixed at 0.0 (middle); and the mutual inclinations relative to the binary plane (right). 

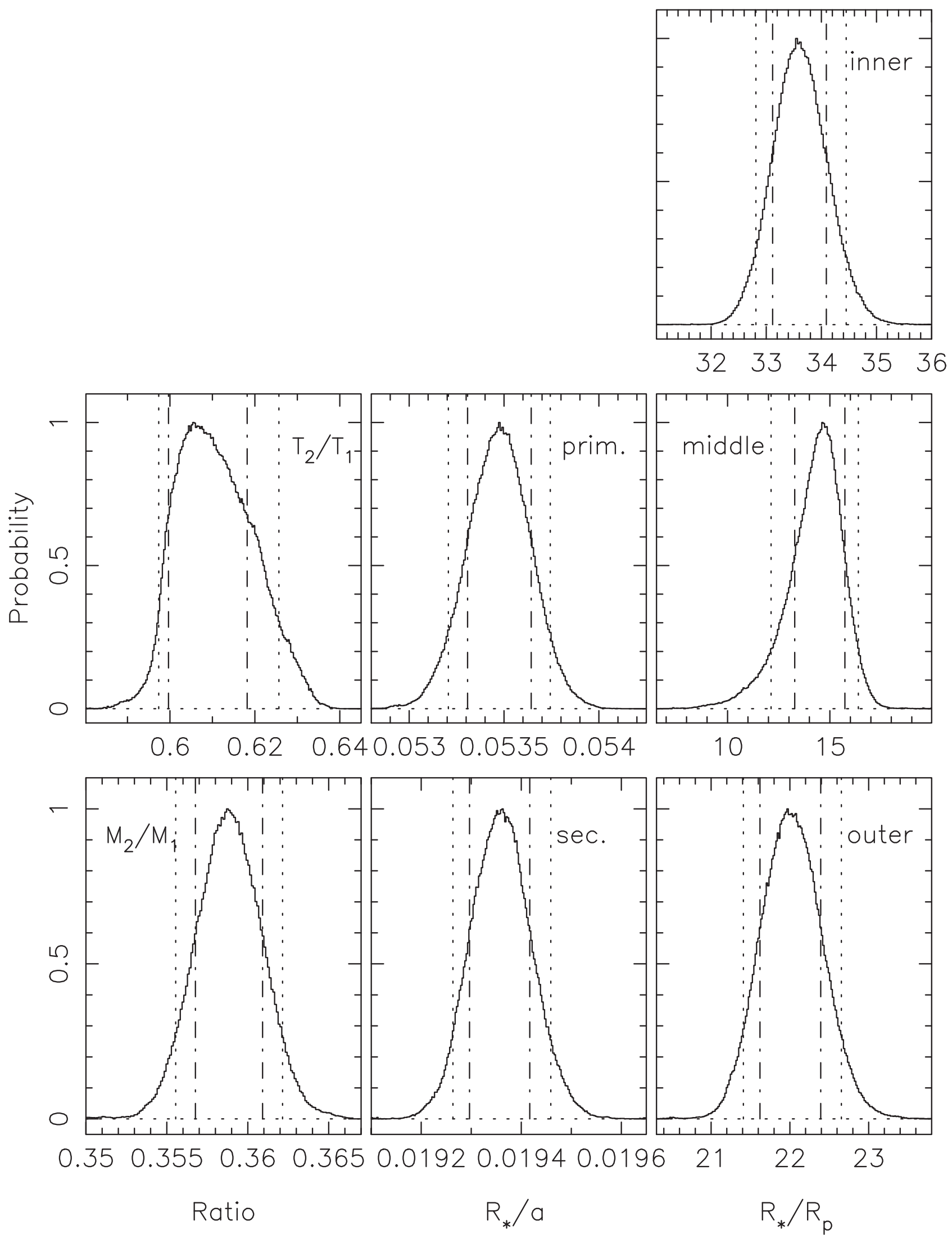

Figure 13. Similar to Figure 10, but showing the posterior distributions of the binary mass and temperature ratios (left), the fractional stellar radii $R_{*} / a($ middle), and the planet radius ratios $R_{*} / R_{p}$ (right). 


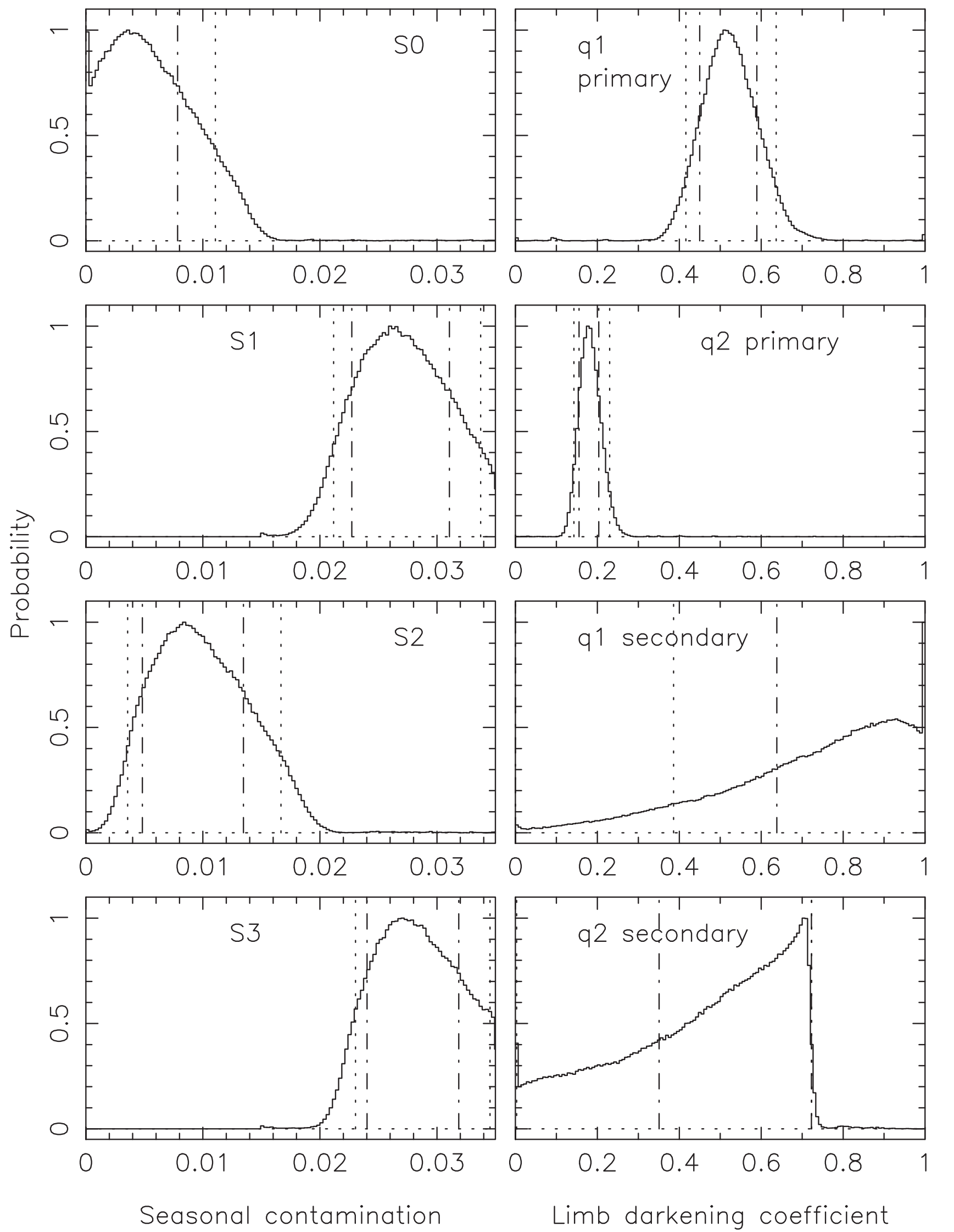

Figure 14. Similar to Figure 10, but showing the posterior distributions of the seasonal contamination parameters (left) and the limb darkening parameters (right). 


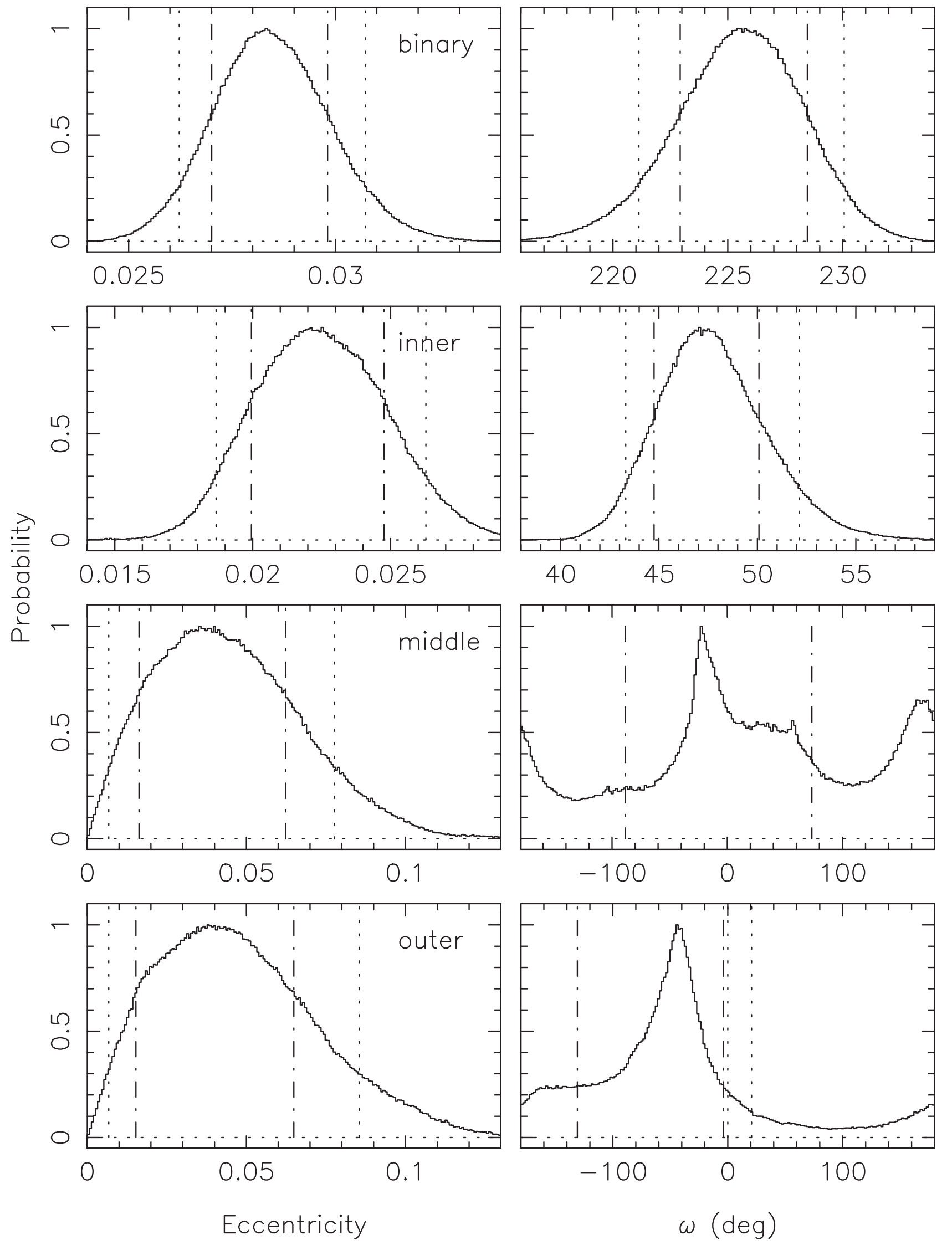

Figure 15. Similar to Figure 10, but showing the posterior distributions of the eccentricities (left) and arguments of periastron (right). 

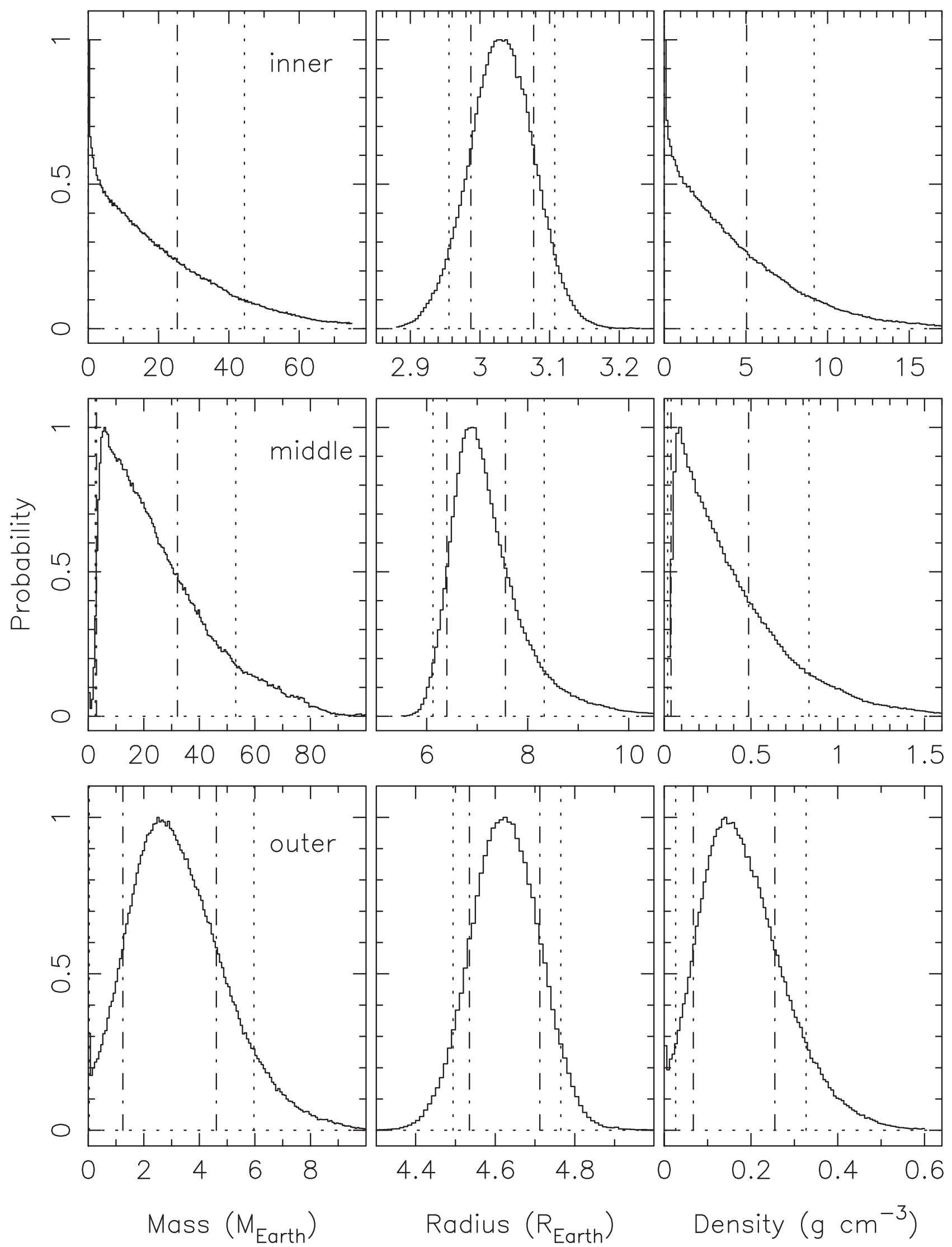

Figure 16. Similar to Figure 10, but showing the posterior distributions of the planet masses (left), the planet radii (middle), and the planet densities (right). 


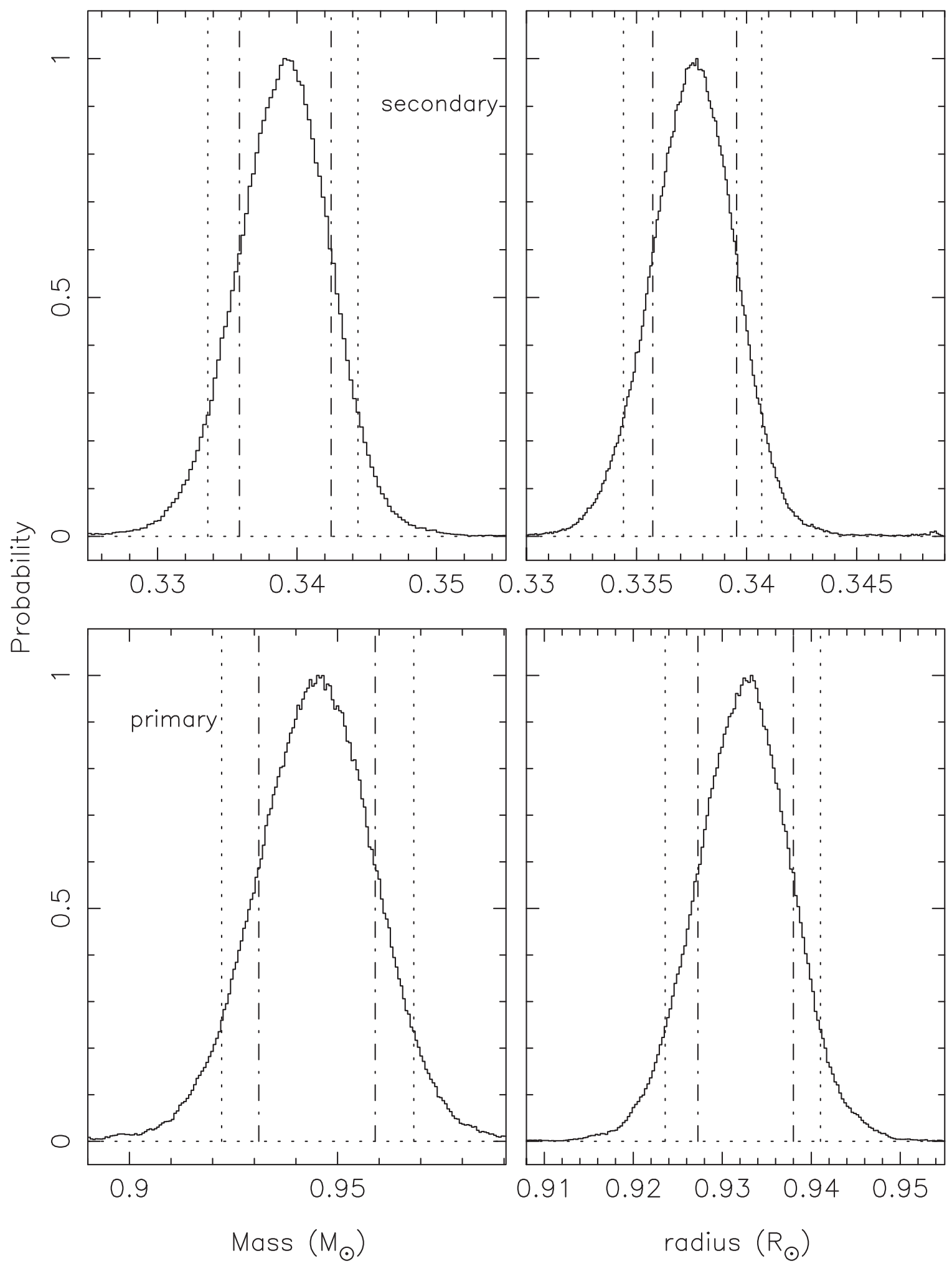

Figure 17. Similar to Figure 10, but showing the posterior distributions of the stellar masses (left) and the stellar radii (right).

precession timescales of the inner, middle, and outer planets to be $\sim 10 \mathrm{yr}, 245 \mathrm{yr}$, and $738 \mathrm{yr}$, respectively (see Figure 23). Figure 24 shows the evolution of the impact parameters (for transits across the primary star) for the three planets. The horizontal dashed lines in the figure denote impact parameters where transits of the primary star could occur. As one might expect, the precessionary motion affects the duration of time for which a given planet can transit relative to our line of sight (Schneider 1994).

We examined the possibility of additional planets within the Kepler-47 system. Before exploring the possibility of the existence of an actual planet, we began by integrating the orbits of a large battery of test particles between the system's planets. The numerical scheme for these simulations used a modified 


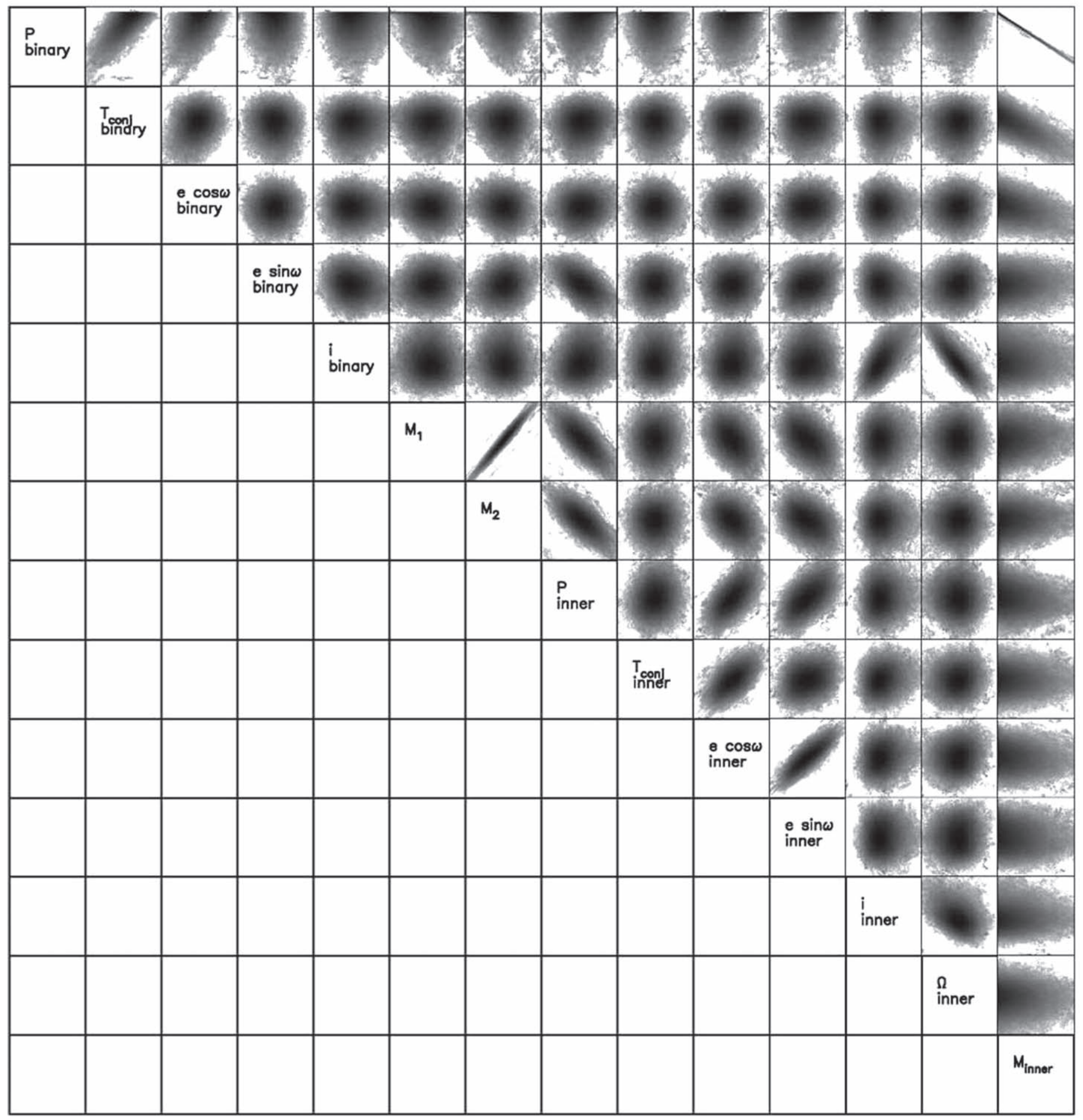

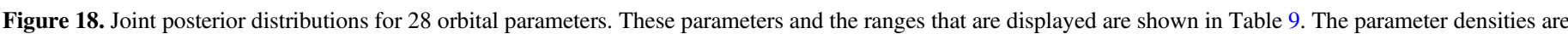

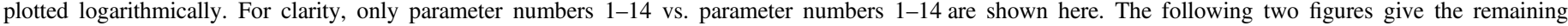
combinations.

version of the orbital integration package, mercury6, that is specifically designed to evaluate the orbits of CBPs efficiently (Chambers et al. 2002). We chose to investigate the test particles initially on coplanar (relative to the binary plane), circular orbits and varied the initial phase of the test particles between $0^{\circ}$ and $360^{\circ}$, in increments of $2^{\circ}$. Additionally, we varied the starting semimajor axis of the test bodies from 0.25 to $1.05 \mathrm{au}$, such that we could evaluate whether non-transiting planets between either the inner-middle pair or the middle-outer pair could be stable for $10 \mathrm{Myr}$. Overall, we integrated test particles corresponding to a grid of $801 \times 181$ initial conditions. From this study, we found that virtually all of the test particles between the middle and outer planets became unstable (experiencing ejection from the system or a collision with another body), indicating that no stable (prograde) planetary orbits exist between these two planets. In other 


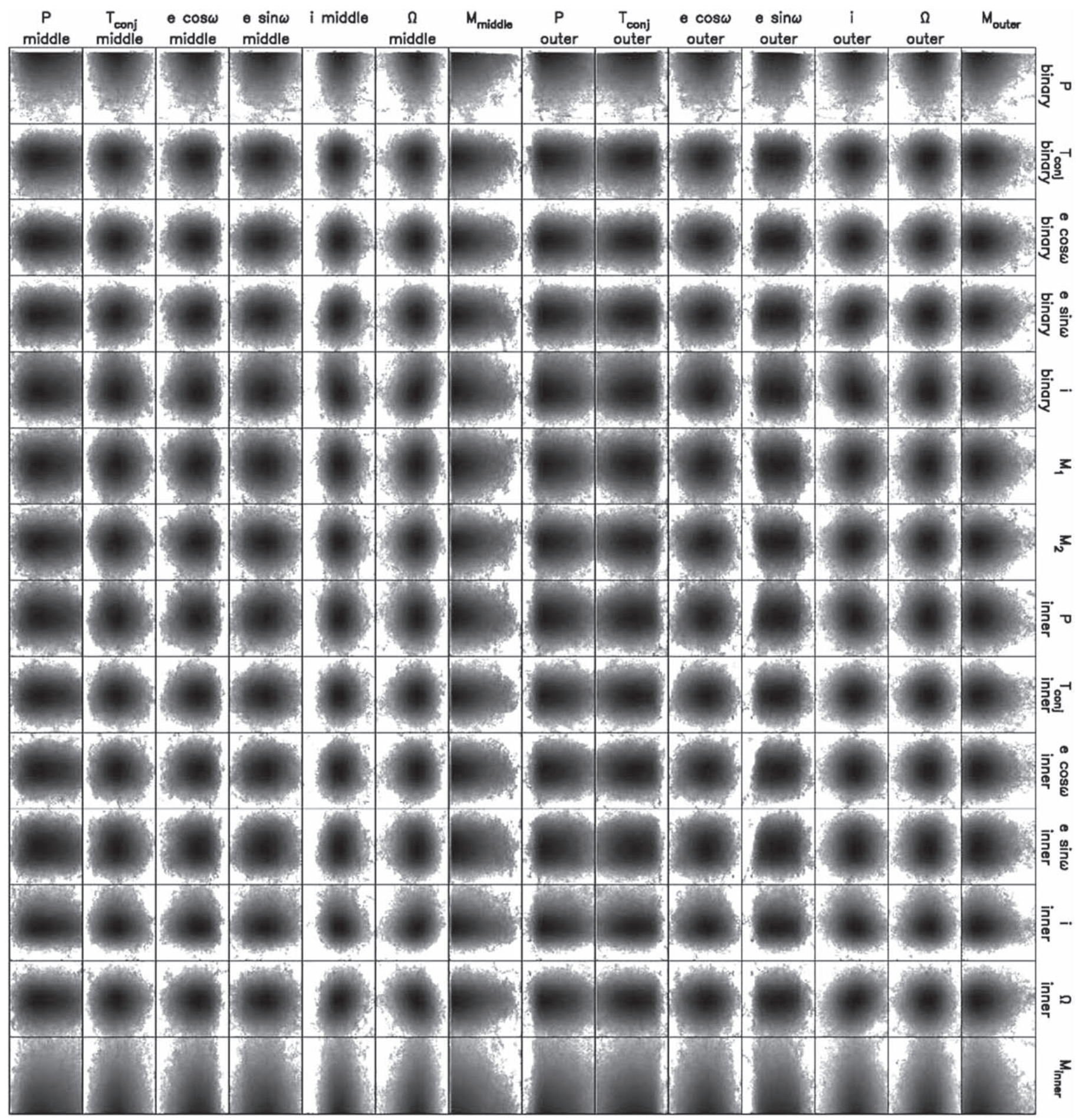

Figure 19. Similar to Figure 18, for parameter numbers $15-28$ vs. parameter numbers $1-14$.

words, the region between the middle and outer planets is dynamically full (i.e., these planets are in a packed orbital configuration). However, there remained a broad region of parameter space where the test particles were stable between the inner and middle planets. Despite this stability, there is insufficient evidence to suggest that a massive body exists, because of the many dynamical interactions that would influence the observations of the transiting planets and thereby alert us to its presence. There are many regions where the test particles' eccentricities were significantly increased due to mean motion resonances with the middle planet and we expect these locations to be eroded over time in a similar manner to how the Kirkwood gaps in the solar system are.

Based on analytical studies, we used the estimate of Chambers et al. (1996), assuming that the binary can be approximated as a single star with a large oblateness, and found that the separation in mutual Hill radii of the inner-middle pair and middle-outer pair to be $32.90 \pm 17.44 R_{H, m}$ and $12.34 \pm 4.43 R_{H, m}$, respectively. These values are well above the critical value of $2 \sqrt{3} \approx 3.46$ for single-star systems 


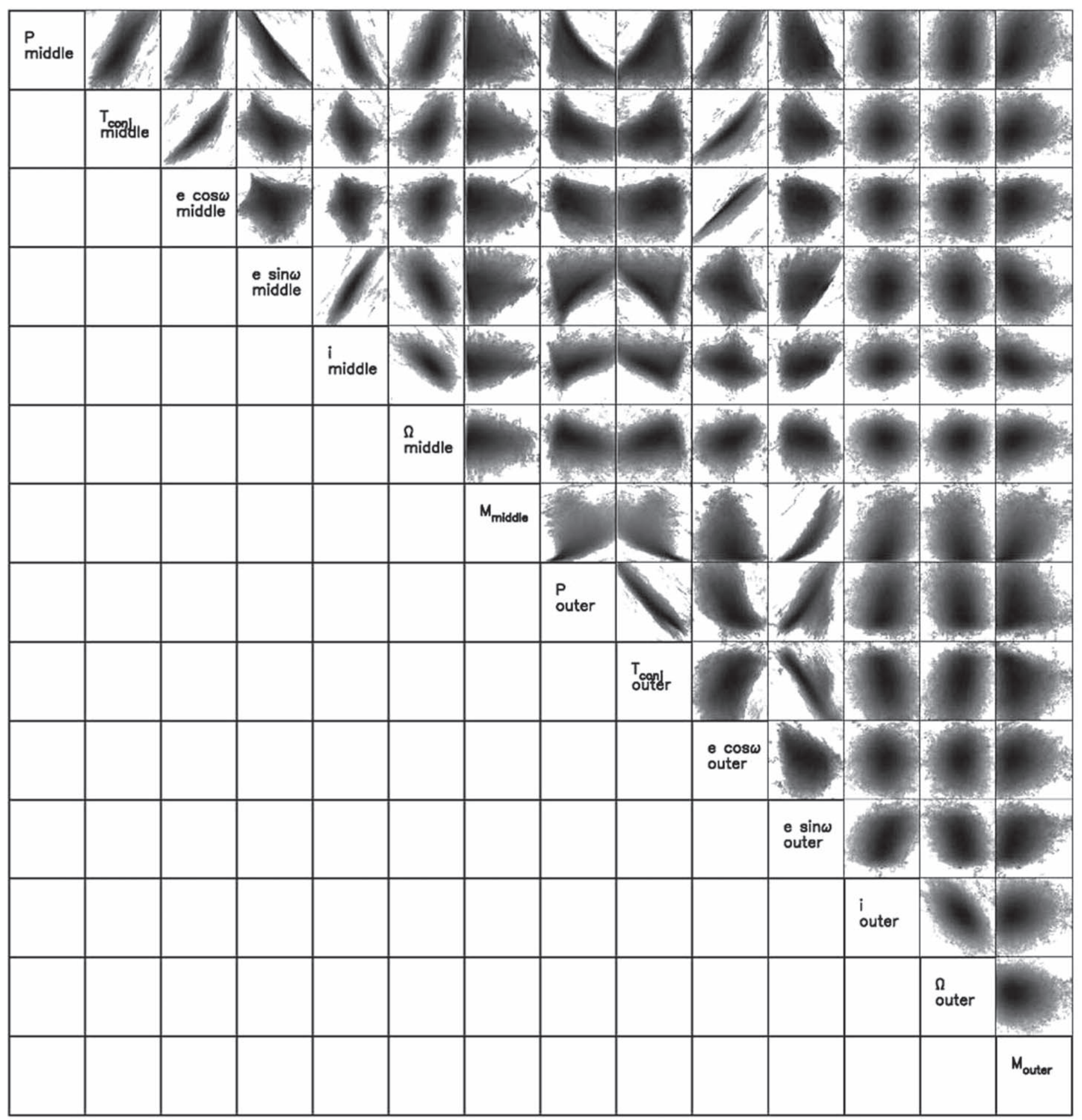

Figure 20. Similar to Figure 18 for parameter numbers $15-28$ vs. parameter numbers $15-28$.

(Gladman 1993) or the critical range of values (5-7) suggested by Kratter \& Shannon (2014) for circumbinary systems. The mutual Hill radii of the middle-outer pair are quite close, even when considering the uncertainties, and this proximity does not allow for any additional planets to orbit between 0.7 and $0.96 \mathrm{au}$. Based on our numerical tests, assuming nominal mass estimates, the middle and outer planets are "packed." This conclusion is supported by other numerical studies (Smith \& Lissauer 2009; Kratter \& Shannon 2014; Quarles \& Lissauer 2018).
We have calculated a dynamical Mean Exponential Growth factor of Nearby Orbits (MEGNO) map (Cincotta et al. 2003; Goździewski et al. 2001) of the middle planet using the best-fit osculating (Jacobian) initial conditions presented in this work. Initial values for the mean anomalies of the binary and each planet were set to zero. The MEGNO factor $\langle Y\rangle$ is a numerical technique to detect and differentiate between aperiodic (chaotic) and quasiperiodic (regular/stable) motion. The computation of the variational vector necessary to calculate MEGNO was done by solving the variational equations of 


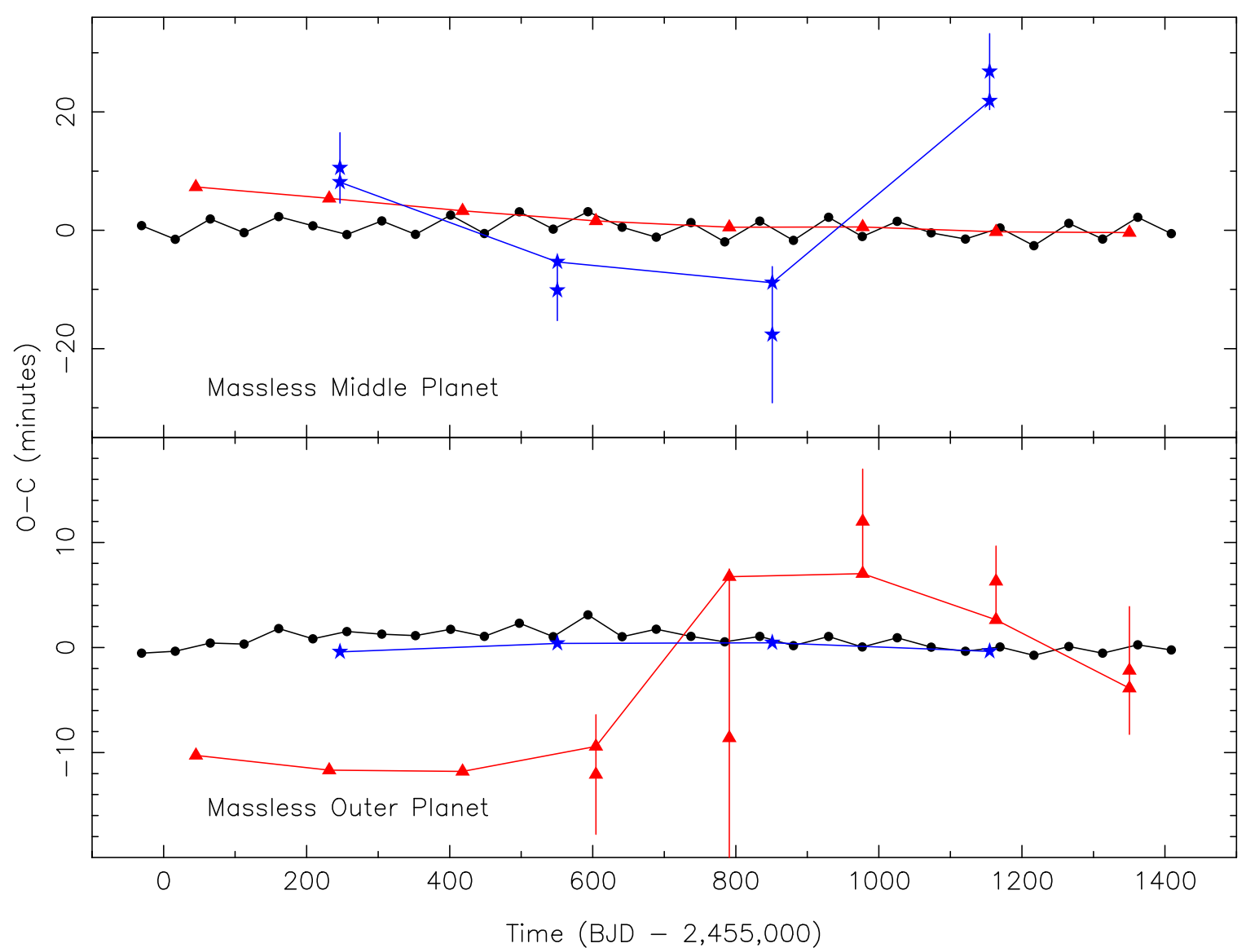

Figure 21. Top: the $O-C$ diagram showing the change in the times of the planet transits when the middle planet is forced to have zero mass (i.e., the times from the overall best-fitting minus the times from the model with the zero-mass middle planet), using black filled circles for the inner planet, blue filled stars for the middle planet, and red filled triangles for the outer planet. The filled stars with error bars are the observed transit times for the outer planet minus the predicted times from the model with the zero-mass middle planet. Bottom: similar to the top, but where the outer planet is forced to have zero mass. The filled triangles with error bars are the observed transit times for the middle planet minus the predicted times from the model with the zero-mass outer planet. The $O-C$ value for the first observed transit (near day 418) is $\approx 41 \pm 9$ minutes, but this value is probably prone to large systematic errors owing to the missing cadence near the middle of the transit.

motion in parallel to the five-body equations of motion. These equations of motion were solved using the accurate adaptive time-step ODEX integrator. ${ }^{19}$ A given integration was terminated once $\langle Y\rangle$ was greater than five. The result of computing MEGNO over a grid in semimajor axis versus eccentricity space is shown in Figure 25. The color coding follows the numerical value of MEGNO attained at the end of the integration. Yellow colors indicate chaotic and a blue $(|\langle Y\rangle-2.0|<0.001)$ color indicate quasiperiodic dynamics of the third planet. The binary and remaining two planets were started at their best-fit osculating Jacobian elements.

Recently, the MEGNO technique was used to study possible positions of the middle planet in the framework of the fivebody problem (Hinse et al. 2015). Compared to that study, the quantitative picture of the semimajor axis versus eccentricity phase-space structure has changed significantly in the present analysis. The main difference is that the eccentricity of the outer planet has decreased from 0.41 to almost zero in the newly found best-fit model. This demonstrates the overall destabilizing effect an eccentricity of 0.41 for the outer planet orbit has, as it would greatly limit the number of possible stable orbits of the middle planet. This is a fine example of the possibility of planet packing as demonstrated in

\footnotetext{
${ }^{19}$ http://www.unige.ch/ hairer/prog/nonstiff/odex.f
}

Kratter \& Shannon (2014). Finally, the location of meanmotion resonances (vertical structures in Figure 25) might change for different initial phases of the planets (when varied within their parameter uncertainties). However, the overall global topology structure of chaotic/quasiperiodic orbits of the third planet would not change dramatically.

While there is no dynamical evidence for an additional planet in the Kepler-47 system, another (low-mass) planet between the inner and middle planets could exist. Also, more planets could have stable orbits sufficiently far outside the orbit of the outer planet. Thus, it would be worthwhile to obtain additional highprecision photometry of Kepler-47 whenever possible, to look for additional transits. From Figure 24, we see that the precession timescales for the three known planets are relatively short, and that the middle and outer planets can transit the primary star for only a small fraction of their precession cycles ( $\sim 23 \%$ and $\sim 12 \%$ of the time for the middle and outer planets, respectively). If there are additional planets in the Kepler-47 system with similar precession cycles, they might precess into view at a later time.

\subsection{Comparison with Stellar Evolution Models}

Our new values for the masses of the stars in Kepler-47 are about $6 \%$ smaller than in the discovery paper, and the new radii are about 3\% smaller than previously reported. We therefore update our analysis and discussion of the evolutionary state of 

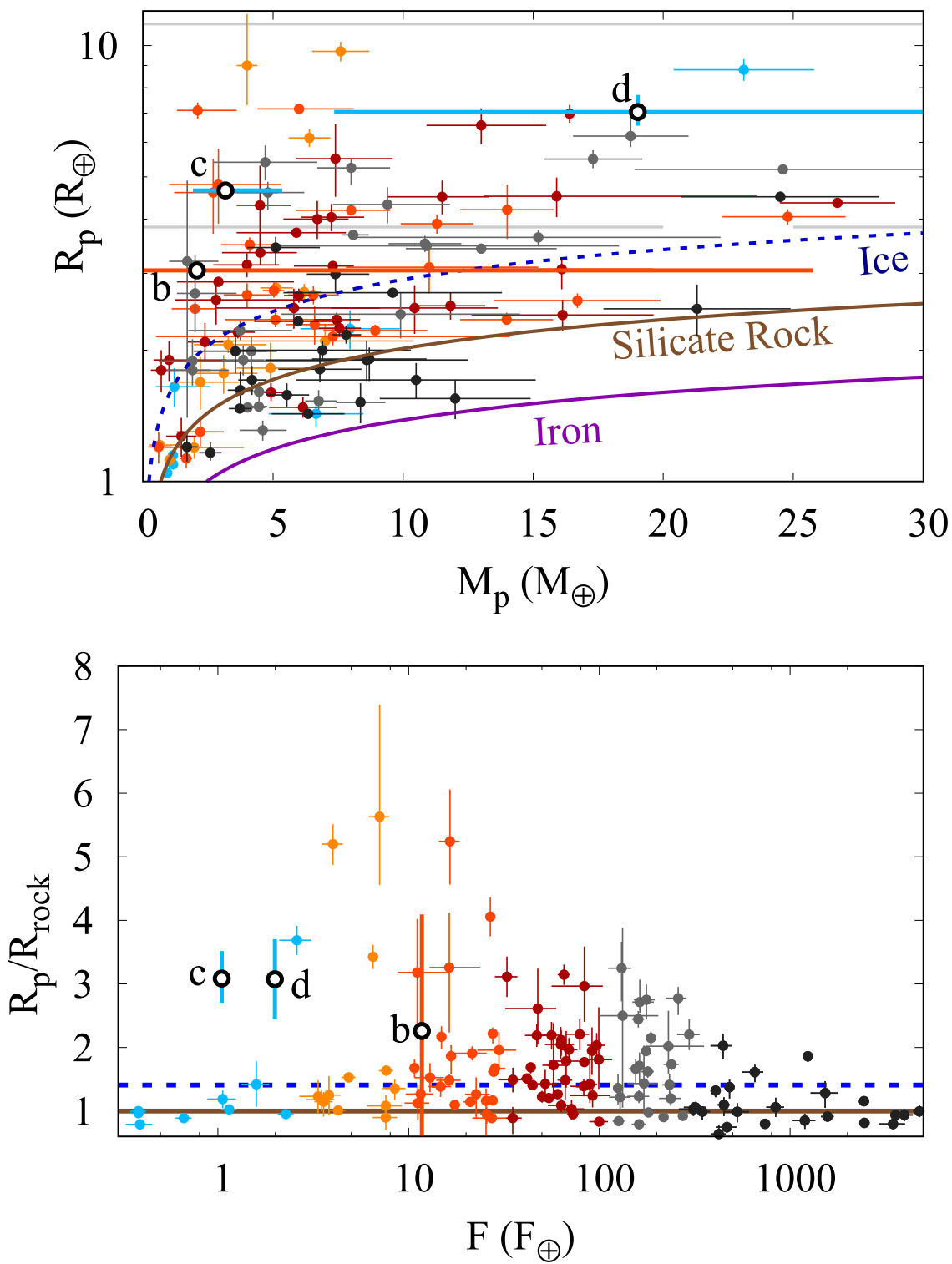

Figure 22. Top: radii and masses for well-measured low-mass exoplanets are plotted, with the Kepler-47 planets shown with open circles along with their $1 \sigma$ error bars. The colors denote the incident flux (S) on each planet relative to the Sun-Earth insolation, ranging from blue for $S<3$ to black for $S>300$ (with orange, red, maroon, and dark gray in-between). For comparison, theoretical curves (Lopez \& Fortney 2014) for planets composed of pure iron, silicate rock, or water are shown. Bottom: the radii of the planets shown in the upper panel, relative to a silicate rock world of the same mass, are plotted against the incident flux the planet receives from its host star. Planets larger (less dense) than a pure-rock planet lie above the value $R_{p} / R_{\text {rock }}=1$. Planets larger than a pure water planet lie above the dashed blue line. These low-density planets must have deep hydrogen and helium atmospheres. Planets that have $R_{p} / R_{\text {rock }}<1$ are denser than pure rock and are probably a mixture of rock and metal, similar to Earth and Venus. The middle and outer planets have significantly larger values of $R_{p} / R_{\text {rock }}$ than other planets with similar lowincidence fluxes.

the Kepler-47 binary. Figure 26 compares the new measurements against Dartmouth models (Dotter et al. 2008) for the nominal spectroscopic metallicity of $[\mathrm{Fe} / \mathrm{H}]=-0.25$ (Orosz et al. 2012b), in the same way as we did previously. There is still an age discrepancy for the primary star: the mass/radius combination yields an age of $3.5 \mathrm{Gyr}$ (solid line in top panel), whereas the mass/temperature combination gives $11.5 \mathrm{Gyr}$. If the radii are given more weight than the temperatures, then the secondary star is now slightly larger than the theoretical predictions, as is seen in many other M dwarfs. As we noted in the discovery paper, the primary temperature derived from color indices points to a higher value than the spectroscopy does (which would actually be consistent with the 3.5 Gyr age).
However, given the uncertainties in the reddening, the spectroscopy is perhaps more trustworthy.

By way of comparison, Figure 27 shows the Dartmouth models for solar metallicity. The primary age from mass/radius is now 4.5 Gyr (solid line), and mass/temperature gives an age consistent with this within the error bar. The secondary radius would be perfectly consistent with the prediction for this metallicity, as would the secondary temperature. However, solar metallicity is formally ruled out by the spectroscopic analysis (Orosz et al. 2012b) at the $3 \sigma$ level, so a scenario where the stars have solar metallicity is unlikely.

We note also that, in its latest data release (DR2), the Gaia mission (Gaia Collaboration et al. 2016, 2018) has provided an accurate parallax for Kepler-47 (source ID 

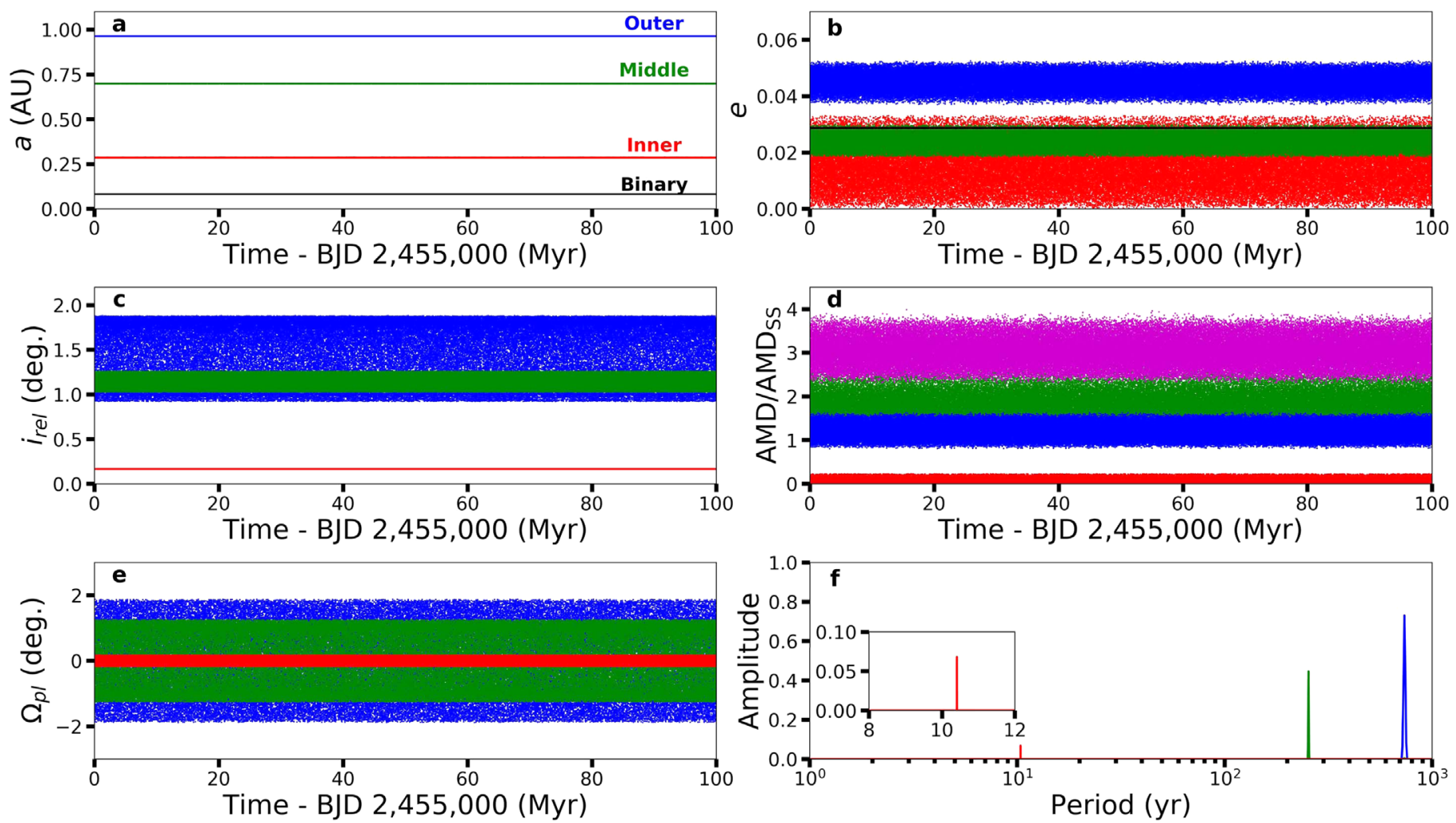

Figure 23. Aspects of the long-term dynamics of the Kepler-47 system. (a) The evolution of the semimajor axes for the four orbits over a $100 \mathrm{Myr}$ simulation. Values for the binary are plotted in black, values for the inner planet's orbit are plotted in red, values for the middle planet's orbit are plotted in green, and values for the outer orbit are plotted in blue. (b) The evolution of the orbital eccentricity $e$ of the four orbits. (c) The evolution of the mutual inclination between the planetary orbits and the binary orbit. (d) The evolution of the AMD relative to the terrestrial planets in the solar system. The violet line shows the total AMD. (e) The evolution of the nodal angle of the planetary orbits. (f) A power spectrum of the variations of the mutual inclination, showing precession timescales of $\sim 10 \mathrm{yr}, 245 \mathrm{yr}$, and $738 \mathrm{yr}$ for the inner, middle, and outer planets, respectively.
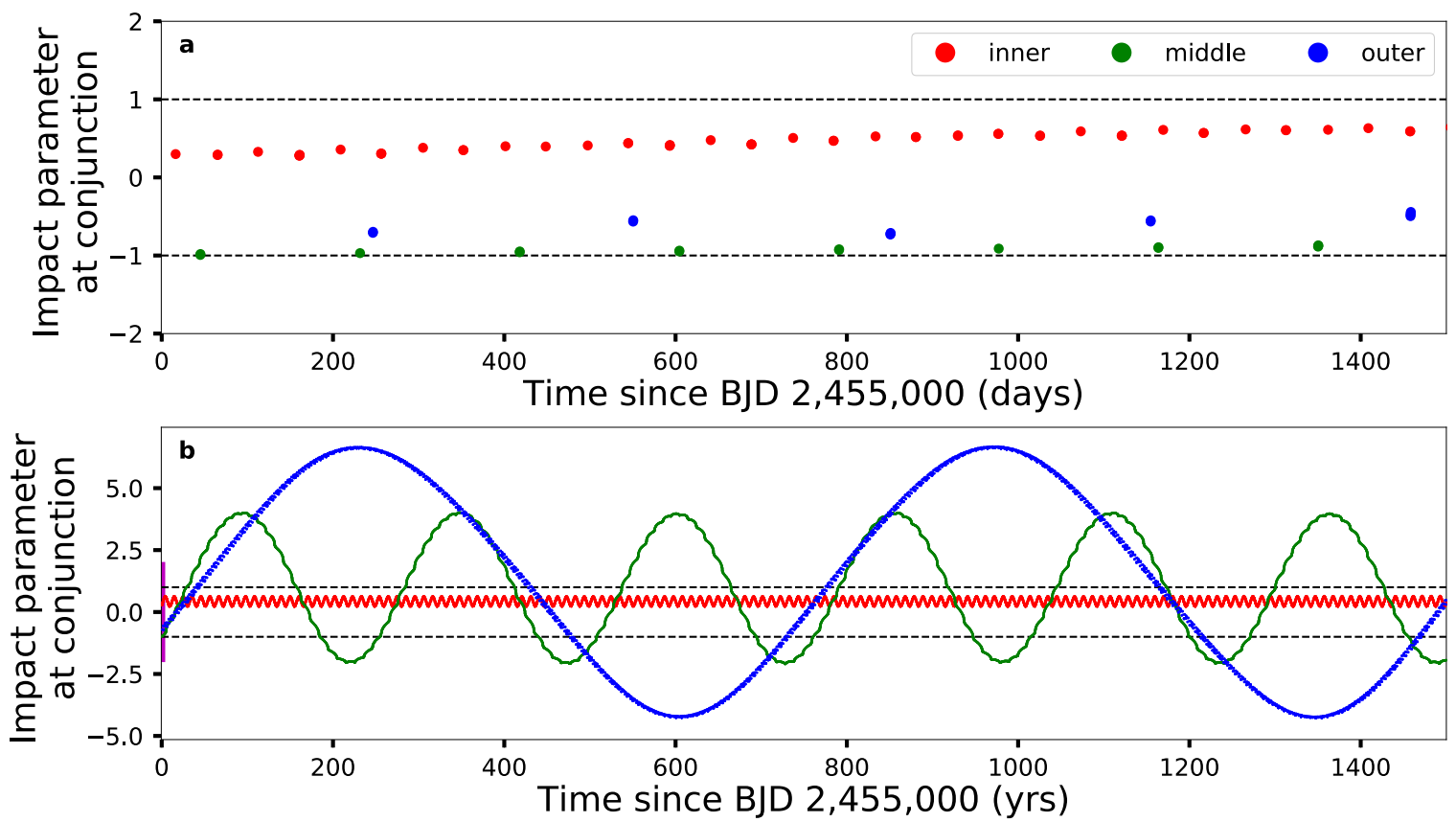

Figure 24. Short-term evolution of future conjunctions. (a) The evolution of the impact parameter $b$ at conjunction with the primary star for the inner (red), middle (green), and outer (blue) planets for the duration of the Kepler mission in days. (b) The evolution of future conjunctions ( $b \lesssim 1$ for transits) with the primary for each planet for the next $1500 \mathrm{yr}$, demonstrating the precession cycles of the orbital planes. 


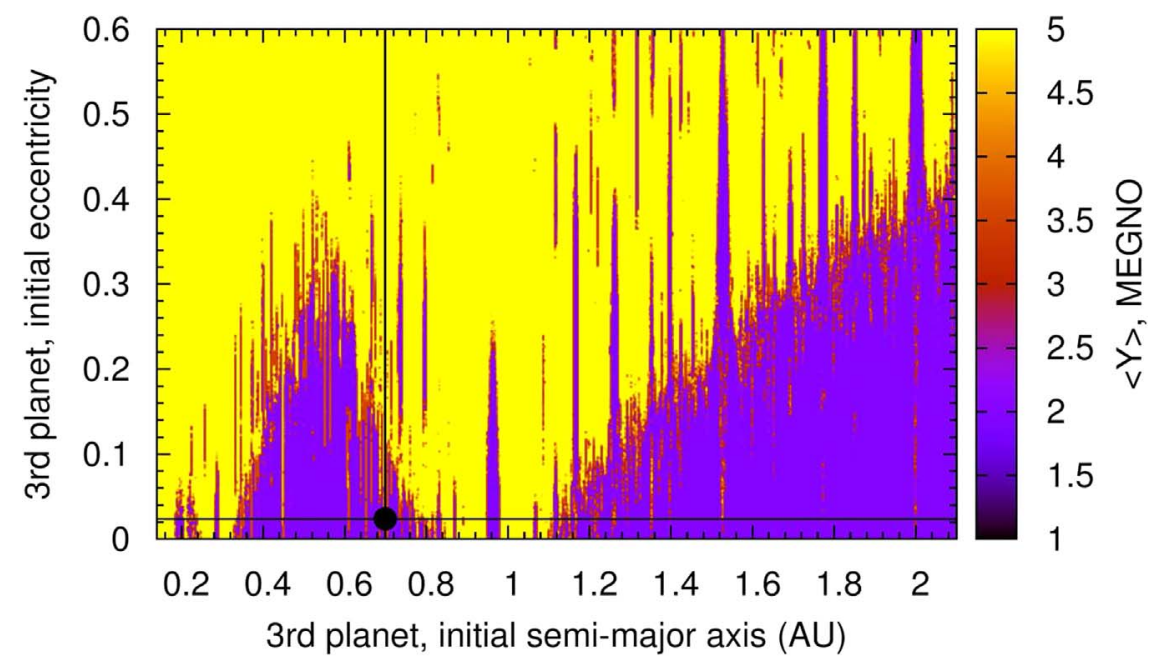

Figure 25. A dynamical MEGNO map for the five-body system is shown. The map shows chaotic (yellow color code)/quasiperiodic (blue color code) regions for a third planet being perturbed by the remaining four bodies (the two stars and the inner and outer planets, with nominal parameter values). The resolution is $N_{x}=600$, $N_{y}=500$ pixels, rendering a total of 30,000 initial conditions. Each orbit is integrated for $5000 \mathrm{yr}$. The location of the middle planet's best-fit osculating element is shown by a filled circle placing it in a region of quasiperiodicity packed between the inner and outer planets.
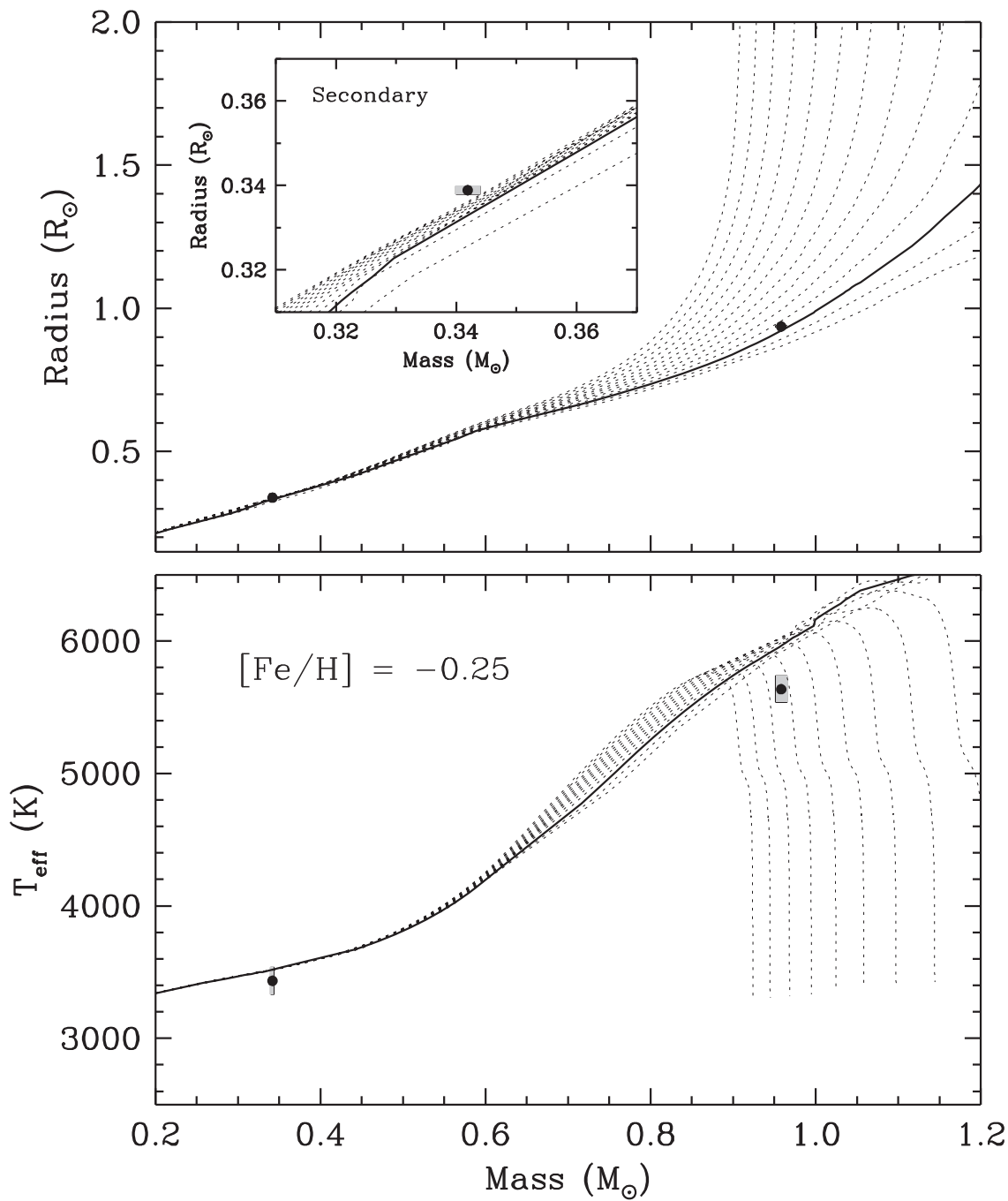

Figure 26. Isochrones from the Dartmouth models (Dotter et al. 2008) corresponding to ages from 1 to $13 \mathrm{Gyr}$ (the oldest isochrone is the steepest one at the highmass end) and metallicity of $[\mathrm{Fe} / \mathrm{H}]=-0.25$ (Orosz et al. 2012b), compared against the measured masses, radii, and temperatures of the stars in Kepler-47. The shaded boxes represent the error bars for the measurements. The top shows the mass-radius plane, with the inset showing a magnified view near the secondary. The bottom shows the mass-temperature diagram. 


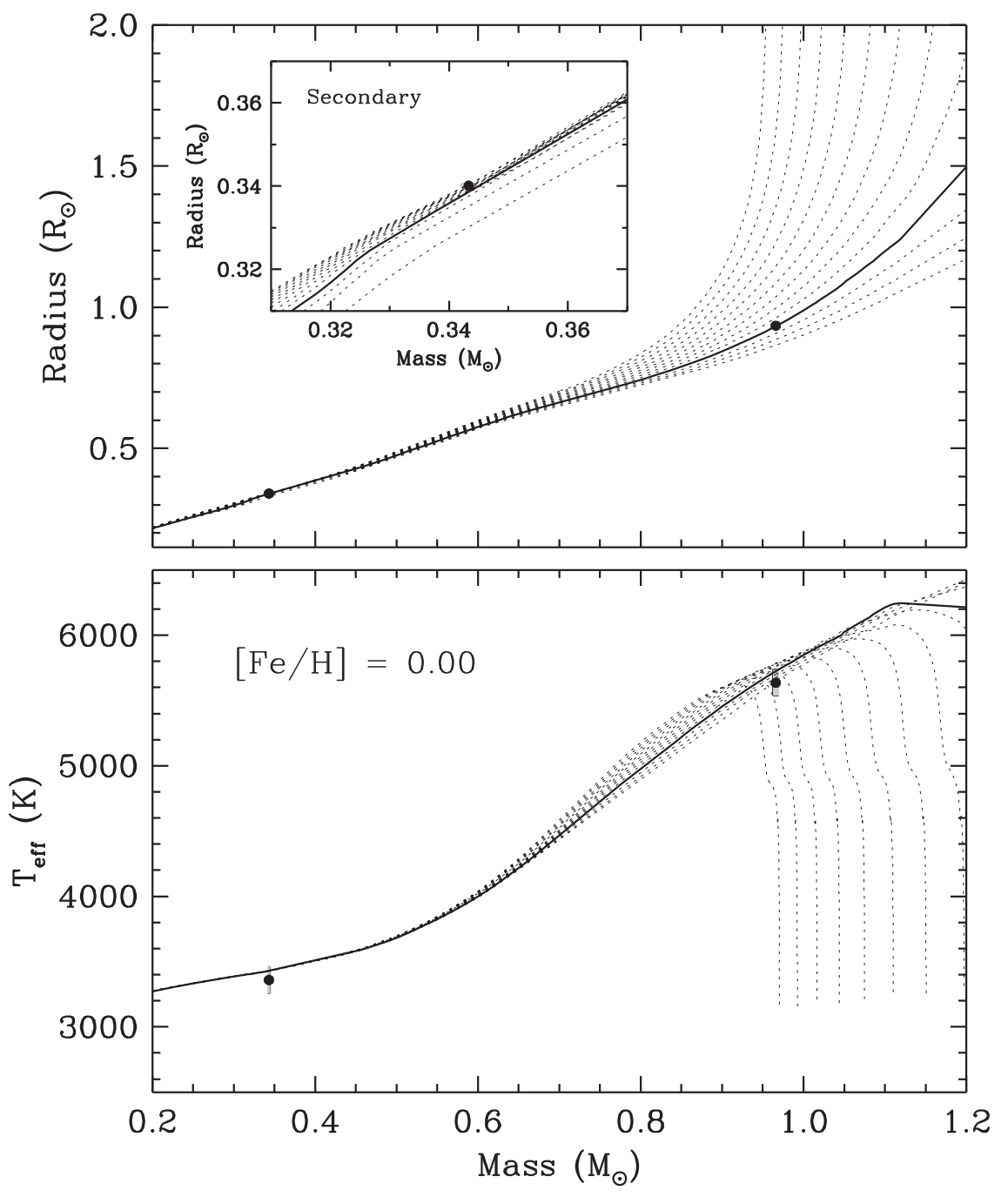

Figure 27. Similar to Figure 26, but for Dartmouth models with solar metallicity.

2080506523540902912) of $\pi=0.948 \pm 0.029$ mas, corresponding to a distance of $1025_{-29.7}^{+31.5}$ pc (Bailer-Jones et al. 2018), that enables a check on our dynamical radius measurement for the primary star. With a reddening estimate to the system of $E$ $(B-V)=0.056 \pm 0.020$ from Green et al. (2015), the assumption that $A_{V}=3.1 E(B-V)$, a bolometric correction from Flower (1996) based on the temperature, and the visual magnitude of the star $(V=15.395 \pm 0.010$, Henden et al. 2015), we obtain $R=0.928 \pm 0.072 R_{\odot}$. This is less precise than our dynamical value of $R=0.936 \pm 0.005 R_{\odot}$, but is in excellent agreement.

\subsection{The Habitable Zone}

The habitable zone (HZ) is usually defined as the region around a star such that the stellar incident flux on the planet (i.e., the insolation) would allow an Earth-like planet with a $\mathrm{CO}_{2} / \mathrm{H}_{2} \mathrm{O} / \mathrm{N}_{2}$ atmosphere to permanently maintain liquid water on its solid surface. The situation is more complicated for a CBP system, in that: (i) there are two stars, typically of different temperatures and luminosities; and (ii) the orbital motion of the stars causes the HZ to "orbit" with the binary. Even for a planet on a purely circular orbit, the planet-star distances are always rapidly changing. The $\mathrm{HZ}$ is no longer static or spherically symmetric.

Therefore, for a CBP system, the definition of the HZ has to be slightly generalized. Here, we define a circumbinary $\mathrm{HZ}$ as the region in space where the time-averaged weighted insolation would allow conditions conducive to liquid water on the surface of an Earth-like planet. The weighted insolation is not simply the total flux incident at the top of the planet's atmosphere (though it reduces to this if one of the stars' flux contribution is negligible compared to the other, as is the case for Kepler-47). Adding the flux from each star is not appropriate because it is not simply a matter of the total energy; the spectral energy distribution is highly relevant. The planet's atmosphere acts to filter the energy received from each star before it reaches the planet's surface, and this filtering is strongly wavelength-dependent. The spectral energy distribution of the light emitted by the star can be reduced to a simple function of the star's effective temperature $\left(T_{\text {eff }}\right)$ to a sufficient level of approximation. Thus, the $T_{\text {eff }}$-weighted sum of the fluxes from each star is used as the total flux received by the planet (Haghighipour \& Kaltenegger 2013). To determine the inner and outer boundaries of the circumbinary HZ, we equate the weighted incident flux with the insolation 


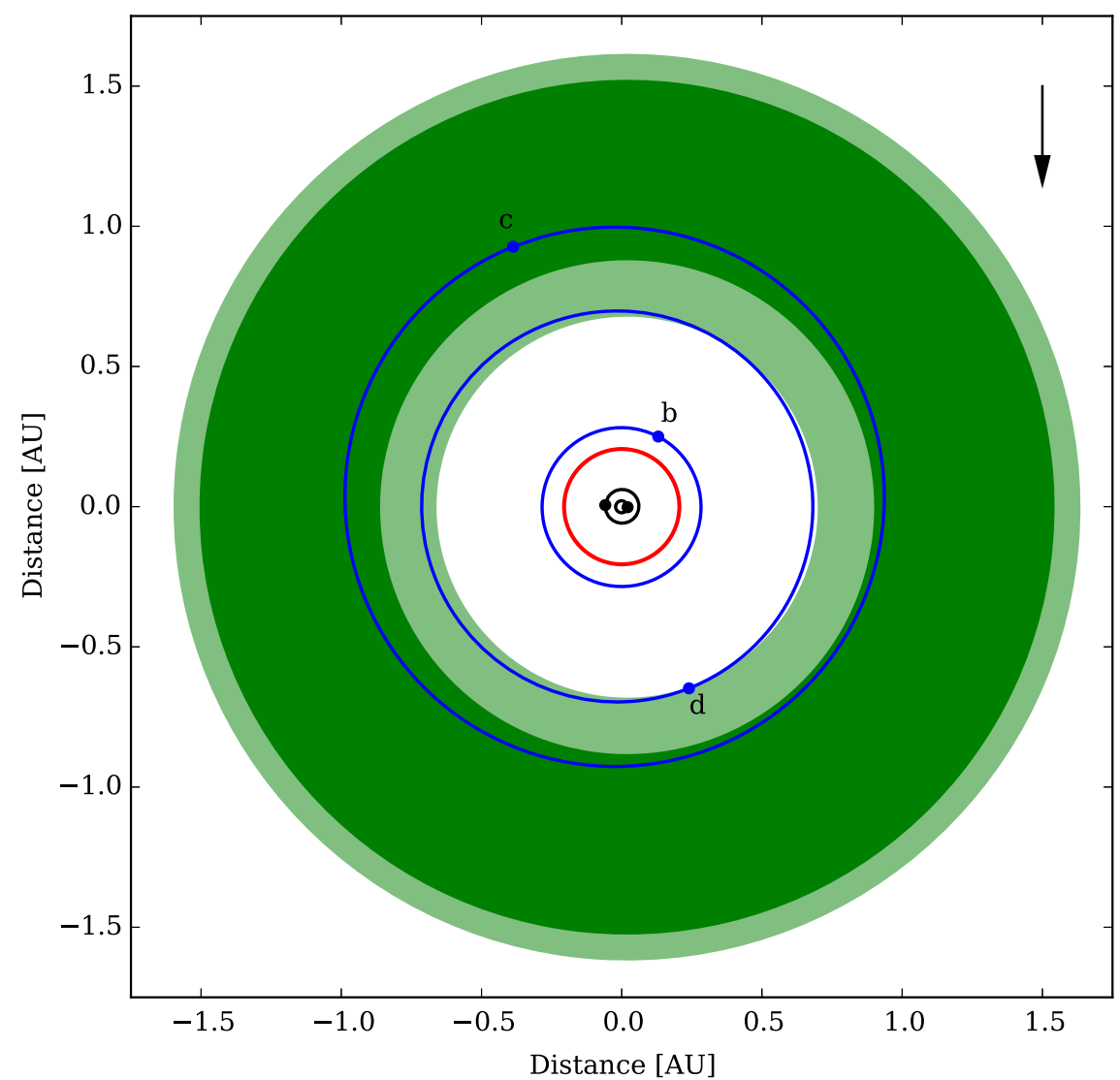

Figure 28. Conservative (dark green) and optimistic (light green) habitable zone regions are shown for the Kepler- 47 system. The red circle shows the critical stability radius (Holman \& Wiegert 1999), interior to which planetary orbits are most likely unstable.

corresponding to those boundaries for a single star as computed by Kopparapu et al. (2013, 2014).

Figure 28 shows a face-on view of the Kepler-47 system. The binary stars are shown in black, the planets in blue, the HZ in green, and the red circle marks the critical instability radius (Holman \& Wiegert 1999). The darker green shaded area corresponds to the conservative $\mathrm{HZ}$ as defined by the runaway greenhouse and maximum greenhouse conditions. The lighter green regions show the optimistic HZ boundaries, as defined by the recent Venus and early Mars conditions (Kopparapu et al. 2013). The arrow shows the direction of the line of sight from the observer to Kepler-47. The sizes of the stars and planets are not to scale, and their locations are for the epoch of the orbital elements presented in Table 5. The outer planet is within the limits of the conservative HZ, and Planet D (the middle planet) skirts the inner boundary of the optimistic HZ. ${ }^{20}$

In Figure 29, we show the total (unweighted) insolation incident at the top of the atmosphere of the middle planet (in blue) and the outer planet (in black) as a function of time. The dotted lines show the limits of the optimistic HZ and the solid green lines mark the limits of the HZ defined by the moist greenhouse condition. The dashed line shows the boundary corresponding to the runaway greenhouse condition, which is also often used as the boundary for the inner edge of the conservative HZ. The three panels show three different timescales. The left panel spans two binary star periods, and the variations in insolation are dominated by the orbital motion

\footnotetext{
${ }^{20}$ An animated version of this figure can be found at http://astro.twam.info/ hz-ptype/.
}

of the primary star. Note the primary and secondary eclipses, as seen from the location of the planets. The middle panel shows one orbital period of the outer planet, and the insolation variations now show the effect of the eccentricities of the planet's orbit. The right-hand panel shows five orbits of the outer planet. The time-averaged total insolation for the outer planet is $S=0.865$ in Sun-Earth units, with an rms variation of $7.2 \%$ about the mean. For the middle planet (d), the timeaveraged insolation is $S=1.67$, with an rms variation of $5.5 \%$. For comparative purposes (only), if one assumes a Bond albedo of 0.34 (similar to that of Jupiter or Saturn; see de Pater \& Lissauer (2015)), and complete redistribution of the incident energy, the equilibrium temperature is $T_{\text {eq }} \approx 241 \mathrm{~K}$ for the outer planet. In the course of this discussion, we must keep in mind that these are low-density planets, with thick $\mathrm{H}, \mathrm{He}$ atmospheres, and therefore not likely to be habitable.

\subsection{Times, Durations, and Impact Parameters of Future Eclipses and Planet Transits}

Ground-based observations of eclipse and transit events would be useful in constraining the dynamical model of the Kepler-47 system. The times of primary and secondary eclipses are well-fit by the linear ephemerides given in Equation (1). Because the times of the planet transits do not follow a linear ephemeris, we computed (using ELC) the times, impact parameters, and depths of future transits (for the years 2019 through 2026) for each planet using the best-fitting model given in Table 5. The uncertainties on these quantities were estimated using sets of initial conditions drawn from the posterior samples of solutions found from the DE-MCMC run, 

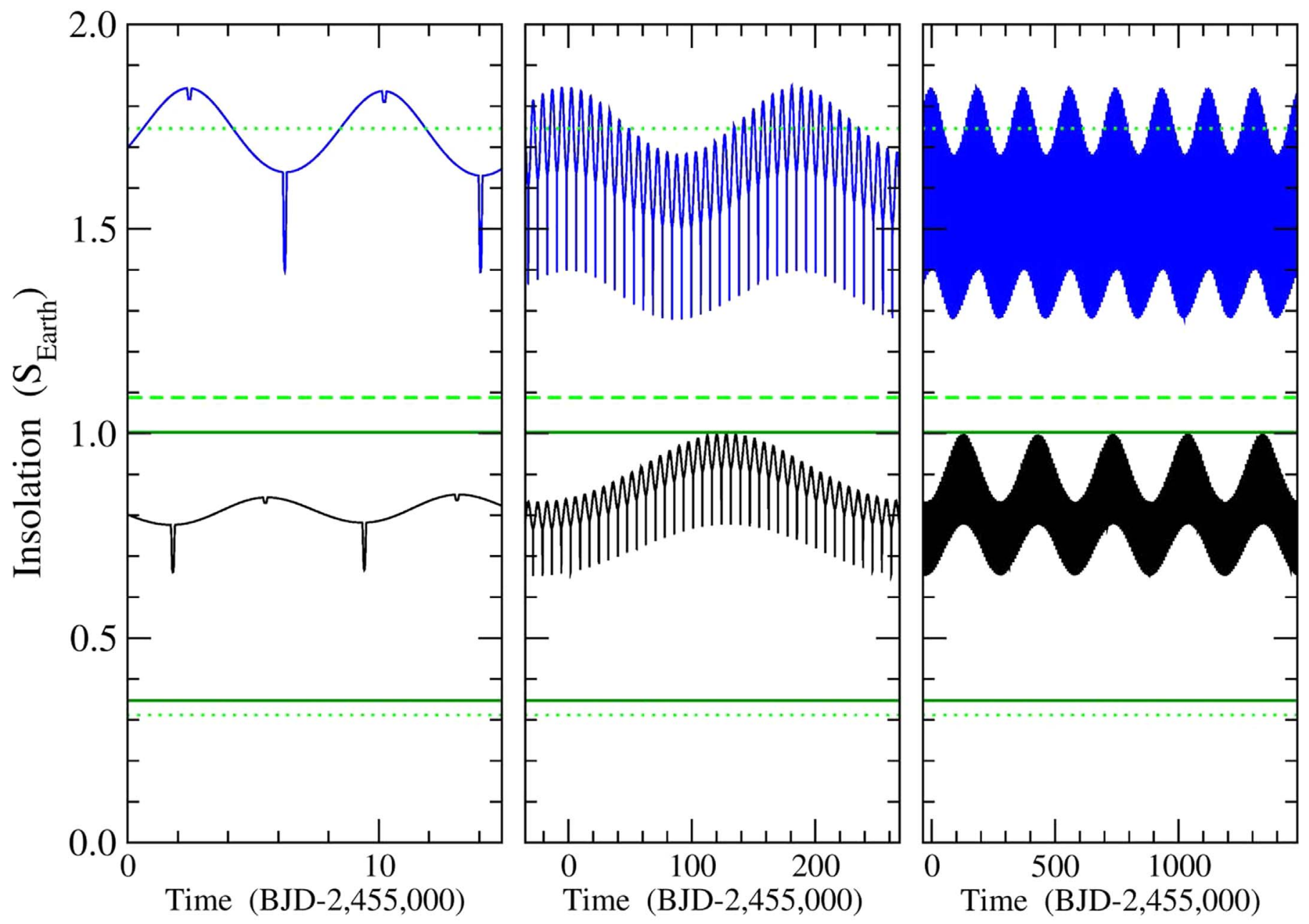

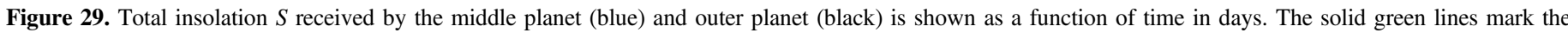

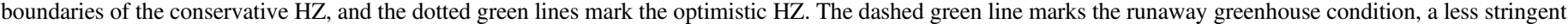

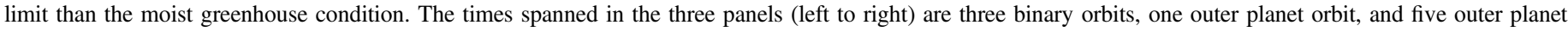
orbits, respectively. The sharp downward spikes show the rapid drop in flux that occurs when the stars eclipse one another.

and are given in Table 10. The formal uncertainties on the predicted times generally vary between about one to two hours for the inner planet, and about 0.75 to two hours for the middle planet and outer planet. With a depth of about $15 \%$, the primary eclipses should be observable by modest-sized ground-based telescopes. On the other hand, owing to the relative faintness of the system $(V=15.395 \pm 0.010$, Henden et al. 2015), the $0.5 \%$ deep secondary eclipses will be more difficult to observe. With depths between about $0.1 \%$ and $0.4 \%$, the transits of three planets will be more challenging still. According to our bestfitting model, the inner planet always transits during its $\sim 10 \mathrm{yr}$ precession cycle, the middle planet transits about $23 \%$ of the time during its $\sim 245 \mathrm{yr}$ precession cycle, and the outer planet transits about $12 \%$ of the time during its $\sim 738 \mathrm{yr}$ precession cycle. We used the REBOUND software (Rein \& Liu 2012) to integrate a set of 368 initial conditions drawn from the posterior sample to estimate the uncertainties on the predicted transit times near the times when the transits stop. We find that the uncertainties of the predicted transit times for the middle planet are about 1.4 days by the time this planet stops transiting in the year $2037.7 \pm 3.4$ (because the transits are grazing before they stop, the predicted stopping date has a much larger spread). Similarly, the uncertainties of the predicted transit times for the outer planet are about 2.1 days by the time this planet stops transiting in the year $2049.1 \pm 6.8$.

\section{Summary}

We have identified a third planet in the Kepler-47 system. Although Kepler-47 d is the largest of the three planets, it was not previously detected because its transits were much weaker in the early Kepler data, as the transit impact parameter was near unity. The precession of its orbital plane is such that the transits are currently growing deeper with time. We modeled the Kepler light curve and radial velocity curve simultaneously using a fivebody photodynamical model with 42 free parameters. The revised system parameters are generally in agreement with the previously published values (Orosz et al. 2012b). The tight constraints placed by the planets on the orbits of the stars enable highly precise measurements of their masses and radii (see Table 6). The stars have masses of $0.957 \pm 0.014$ and $0.342 \pm 0.003 M_{\odot}$, and radii of $0.936 \pm 0.005$ and $0.338 \pm 0.002 R_{\odot}$, consistent with an age of 3.5-11.5 Gyrs and slightly favoring an age in the younger portion of its range. Uncertainties in the planet masses have improved by over an order of magnitude compared with the 2012 discovery paper (Orosz et al. 2012b). The $1 \sigma$ mass estimates for 
Table 10

Times, Durations, and Impact Parameters of Future Planet Transits

\begin{tabular}{|c|c|c|c|c|c|c|}
\hline BJD-2,455,000 & Year & Month & Day & UT & $\begin{array}{c}\text { Impact } \\
\text { Parameter }\end{array}$ & $\begin{array}{c}\text { Duration } \\
(\mathrm{hr})\end{array}$ \\
\hline \multicolumn{7}{|l|}{ Inner Planet } \\
\hline$\overline{3569.12508} \pm 0.06896$ & 2019 & Mar & 26 & 15:00:06.6 & $0.269 \pm 0.034$ & $4.12 \pm 0.07$ \\
\hline $3618.07766 \pm 0.10808$ & 2019 & May & 14 & $13: 51: 49.8$ & $0.245 \pm 0.027$ & $6.66 \pm 0.30$ \\
\hline $3665.39077 \pm 0.06593$ & 2019 & Jun & 30 & $21: 22: 42.5$ & $0.286 \pm 0.030$ & $3.81 \pm 0.04$ \\
\hline $3713.68886 \pm 0.18417$ & 2019 & Aug & 18 & $04: 31: 57.6$ & $0.233 \pm 0.023$ & $10.88 \pm 0.33$ \\
\hline $3761.67854 \pm 0.06705$ & 2019 & Oct & 5 & $04: 17: 05.5$ & $0.303 \pm 0.025$ & $3.77 \pm 0.03$ \\
\hline $3809.11491 \pm 0.14101$ & 2019 & Nov & 21 & $14: 45: 28.6$ & $0.252 \pm 0.023$ & $7.74 \pm 0.54$ \\
\hline $3857.95365 \pm 0.07213$ & 2020 & Jan & 9 & $10: 53: 15.4$ & $0.317 \pm 0.022$ & $3.98 \pm 0.06$ \\
\hline $3905.07251 \pm 0.09442$ & 2020 & $\mathrm{Feb}$ & 25 & $13: 44: 24.5$ & $0.291 \pm 0.021$ & $5.10 \pm 0.18$ \\
\hline $3954.17620 \pm 0.08347$ & 2020 & Apr & 14 & $16: 13: 43.6$ & $0.329 \pm 0.023$ & $4.54 \pm 0.13$ \\
\hline $4001.25330 \pm 0.07808$ & 2020 & May & 31 & $18: 04: 45.0$ & $0.330 \pm 0.021$ & $4.13 \pm 0.07$ \\
\hline $4050.26982 \pm 0.10916$ & 2020 & Jul & 19 & $18: 28: 32.1$ & $0.335 \pm 0.024$ & $5.92 \pm 0.30$ \\
\hline $4097.51864 \pm 0.07189$ & 2020 & Sep & 5 & $00: 26: 50.1$ & $0.367 \pm 0.022$ & $3.72 \pm 0.03$ \\
\hline $4146.02872 \pm 0.17570$ & 2020 & Oct & 23 & $12: 41: 21.0$ & $0.333 \pm 0.024$ & $9.51 \pm 0.68$ \\
\hline $4193.81227 \pm 0.07070$ & 2020 & Dec & 10 & $07: 29: 40.1$ & $0.402 \pm 0.023$ & $3.60 \pm 0.04$ \\
\hline $4241.40816 \pm 0.16954$ & 2021 & Jan & 26 & $21: 47: 44.6$ & $0.349 \pm 0.024$ & $8.56 \pm 0.63$ \\
\hline $4290.10058 \pm 0.07352$ & 2021 & Mar & 16 & $14: 24: 49.9$ & $0.431 \pm 0.022$ & $3.70 \pm 0.07$ \\
\hline $4337.27273 \pm 0.10477$ & 2021 & May & 02 & $18: 32: 43.8$ & $0.395 \pm 0.022$ & $5.28 \pm 0.23$ \\
\hline $4386.34736 \pm 0.08170$ & 2021 & Jun & 20 & $20: 20: 11.5$ & $0.453 \pm 0.024$ & $4.09 \pm 0.11$ \\
\hline $4433.42044 \pm 0.08192$ & 2021 & Aug & 6 & $22: 05: 26.2$ & $0.445 \pm 0.021$ & $4.07 \pm 0.08$ \\
\hline $4482.49229 \pm 0.10084$ & 2021 & Sep & 24 & $23: 48: 53.6$ & $0.466 \pm 0.023$ & $5.07 \pm 0.22$ \\
\hline $4529.67276 \pm 0.07279$ & 2021 & Nov & 11 & $04: 08: 46.6$ & $0.489 \pm 0.020$ & $3.54 \pm 0.03$ \\
\hline $4578.38021 \pm 0.15083$ & 2021 & Dec & 29 & $21: 07: 30.2$ & $0.467 \pm 0.023$ & $7.72 \pm 0.56$ \\
\hline $4625.96328 \pm 0.06974$ & 2022 & Feb & 15 & 11:07:07.1 & $0.527 \pm 0.017$ & $3.34 \pm 0.04$ \\
\hline $4673.77625 \pm 0.19135$ & 2022 & Apr & 04 & $06: 37: 48.3$ & $0.473 \pm 0.018$ & $9.29 \pm 0.45$ \\
\hline $4722.25528 \pm 0.07085$ & 2022 & May & 22 & $18: 07: 36.1$ & $0.555 \pm 0.013$ & $3.35 \pm 0.05$ \\
\hline $4769.49523 \pm 0.11299$ & 2022 & Jul & 8 & $23: 53: 07.8$ & $0.515 \pm 0.013$ & $5.45 \pm 0.29$ \\
\hline $4818.51498 \pm 0.07680$ & 2022 & Aug & 27 & $00: 21: 34.2$ & $0.571 \pm 0.010$ & $3.63 \pm 0.07$ \\
\hline $4865.58850 \pm 0.08351$ & 2022 & Oct & 13 & $02: 07: 26.6$ & $0.559 \pm 0.010$ & $3.95 \pm 0.10$ \\
\hline $4914.69024 \pm 0.09165$ & 2022 & Dec & 1 & $04: 33: 56.9$ & $0.576 \pm 0.009$ & $4.38 \pm 0.15$ \\
\hline $4961.81627 \pm 0.07225$ & 2023 & Jan & 17 & $07: 35: 25.7$ & $0.595 \pm 0.008$ & $3.34 \pm 0.04$ \\
\hline $5010.65834 \pm 0.13025$ & 2023 & Mar & 7 & $03: 48: 00.2$ & $0.569 \pm 0.007$ & $6.37 \pm 0.34$ \\
\hline $5058.09452 \pm 0.06831$ & 2023 & Apr & 23 & $14: 16: 06.2$ & $0.620 \pm 0.009$ & $3.10 \pm 0.03$ \\
\hline $5106.12188 \pm 0.19939$ & 2023 & Jun & 10 & $14: 55: 30.0$ & $0.562 \pm 0.008$ & $9.46 \pm 0.27$ \\
\hline $5154.38086 \pm 0.06892$ & 2023 & Jul & 28 & $21: 08: 26.2$ & $0.632 \pm 0.011$ & $3.10 \pm 0.03$ \\
\hline $5201.68566 \pm 0.12314$ & 2023 & Sep & 14 & $04: 27: 20.8$ & $0.589 \pm 0.012$ & $5.65 \pm 0.40$ \\
\hline $5250.64114 \pm 0.07437$ & 2023 & Nov & 2 & $03: 23: 14.8$ & $0.630 \pm 0.021$ & $3.37 \pm 0.06$ \\
\hline $5297.71619 \pm 0.08725$ & 2023 & Dec & 19 & $05: 11: 18.8$ & $0.618 \pm 0.018$ & $3.92 \pm 0.17$ \\
\hline $5346.82635 \pm 0.08815$ & 2024 & Feb & 6 & $07: 49: 56.9$ & $0.615 \pm 0.028$ & $4.06 \pm 0.10$ \\
\hline $5393.91612 \pm 0.07470$ & 2024 & Mar & 24 & 09:59:12.8 & $0.635 \pm 0.024$ & $3.28 \pm 0.12$ \\
\hline $5442.82740 \pm 0.12356$ & 2024 & May & 12 & $07: 51: 27.2$ & $0.590 \pm 0.034$ & $5.84 \pm 0.29$ \\
\hline $5490.17822 \pm 0.07079$ & 2024 & Jun & 28 & $16: 16: 37.8$ & $0.637 \pm 0.038$ & $3.07 \pm 0.11$ \\
\hline $5538.33935 \pm 0.20718$ & 2024 & Aug & 15 & $20: 08: 39.8$ & $0.568 \pm 0.039$ & $9.59 \pm 0.27$ \\
\hline $5586.45447 \pm 0.07203$ & 2024 & Oct & 2 & $22: 54: 26.1$ & $0.626 \pm 0.046$ & $3.12 \pm 0.11$ \\
\hline $5633.79496 \pm 0.13951$ & 2024 & Nov & 19 & $07: 04: 44.3$ & $0.581 \pm 0.046$ & $6.05 \pm 0.54$ \\
\hline $5682.70773 \pm 0.07857$ & 2025 & Jan & 7 & $04: 59: 07.7$ & $0.601 \pm 0.052$ & $3.44 \pm 0.13$ \\
\hline $5729.77921 \pm 0.09714$ & 2025 & Feb & 23 & $06: 42: 03.4$ & $0.591 \pm 0.053$ & $4.13 \pm 0.28$ \\
\hline $5778.88977 \pm 0.09420$ & 2025 & Apr & 13 & $09: 21: 15.7$ & $0.564 \pm 0.057$ & $4.20 \pm 0.15$ \\
\hline $5825.95882 \pm 0.08342$ & 2025 & May & 30 & 11:00:42.0 & $0.586 \pm 0.060$ & $3.48 \pm 0.19$ \\
\hline $5874.89271 \pm 0.13339$ & 2025 & Jul & 18 & $09: 25: 30.1$ & $0.521 \pm 0.061$ & $6.06 \pm 0.32$ \\
\hline $5922.20903 \pm 0.07985$ & 2025 & Sep & 3 & 17:01:00.3 & $0.567 \pm 0.065$ & $3.29 \pm 0.16$ \\
\hline $5970.40676 \pm 0.23356$ & 2025 & Oct & 21 & $21: 45: 44.1$ & $0.487 \pm 0.065$ & $10.24 \pm 0.34$ \\
\hline $6018.47654 \pm 0.08223$ & 2025 & Dec & 8 & $23: 26: 13.1$ & $0.536 \pm 0.055$ & $3.39 \pm 0.14$ \\
\hline $6065.81849 \pm 0.16443$ & 2026 & Jan & 25 & $07: 38: 37.9$ & $0.491 \pm 0.057$ & $6.57 \pm 0.67$ \\
\hline $6114.72278 \pm 0.09079$ & 2026 & Mar & 15 & $05: 20: 47.8$ & $0.496 \pm 0.057$ & $3.75 \pm 0.15$ \\
\hline $6161.78773 \pm 0.11442$ & 2026 & May & 1 & $06: 54: 19.5$ & $0.488 \pm 0.059$ & $4.49 \pm 0.31$ \\
\hline $6210.89773 \pm 0.11007$ & 2026 & Jun & 19 & $09: 32: 44.3$ & $0.448 \pm 0.057$ & $4.56 \pm 0.15$ \\
\hline $6257.96094 \pm 0.09876$ & 2026 & Aug & 5 & $11: 03: 44.9$ & $0.470 \pm 0.061$ & $3.79 \pm 0.19$ \\
\hline $6306.89174 \pm 0.15754$ & 2026 & Sep & 23 & $09: 24: 06.5$ & $0.397 \pm 0.060$ & $6.55 \pm 0.40$ \\
\hline $6354.20783 \pm 0.09504$ & 2026 & Nov & 9 & $16: 59: 16.2$ & $0.443 \pm 0.060$ & $3.59 \pm 0.14$ \\
\hline $6402.38804 \pm 0.27883$ & 2026 & Dec & 27 & $21: 18: 46.6$ & $0.360 \pm 0.059$ & $10.99 \pm 0.29$ \\
\hline \multicolumn{7}{|l|}{ Middle Planet } \\
\hline$\overline{3590.46599 \pm} 0.01449$ & 2019 & Apr & 16 & $23: 11: 01.3$ & $0.381 \pm 0.031$ & $5.02 \pm 0.05$ \\
\hline
\end{tabular}


Table 10

(Continued)

\begin{tabular}{|c|c|c|c|c|c|c|}
\hline BJD-2,455,000 & Year & Month & Day & UT & $\begin{array}{c}\text { Impact } \\
\text { Parameter }\end{array}$ & $\begin{array}{l}\text { Duration } \\
\text { (hr) }\end{array}$ \\
\hline $3776.89067 \pm 0.01533$ & 2019 & Oct & 20 & $09: 22: 33.7$ & $0.362 \pm 0.032$ & $5.05 \pm 0.04$ \\
\hline $3963.31897 \pm 0.01657$ & 2020 & Apr & 23 & $19: 39: 19.0$ & $0.343 \pm 0.033$ & $5.15 \pm 0.04$ \\
\hline $4149.75504 \pm 0.01850$ & 2020 & Oct & 27 & $06: 07: 15.6$ & $0.326 \pm 0.034$ & $5.32 \pm 0.04$ \\
\hline $4336.19874 \pm 0.02071$ & 2021 & May & 1 & $16: 46: 11.3$ & $0.309 \pm 0.034$ & $5.60 \pm 0.04$ \\
\hline $4522.65635 \pm 0.02359$ & 2021 & Nov & 4 & 03:45:08.9 & $0.292 \pm 0.035$ & $6.04 \pm 0.05$ \\
\hline $4709.13842 \pm 0.02774$ & 2022 & May & 9 & $15: 19: 19.7$ & $0.275 \pm 0.037$ & $6.72 \pm 0.09$ \\
\hline $4895.65032 \pm 0.03401$ & 2022 & Nov & 12 & $03: 36: 27.5$ & $0.256 \pm 0.038$ & $7.84 \pm 0.16$ \\
\hline $5082.21936 \pm 0.04505$ & 2023 & May & 17 & $17: 15: 52.9$ & $0.232 \pm 0.039$ & $9.97 \pm 0.35$ \\
\hline $5268.92328 \pm 0.07347$ & 2023 & Nov & 20 & 10:09:31.0 & $0.199 \pm 0.041$ & $15.74 \pm 1.35$ \\
\hline $5456.14904 \pm 0.20665$ & 2024 & May & 25 & $15: 34: 37.5$ & $0.119 \pm 0.045$ & $34.28 \pm 0.83$ \\
\hline $5643.63396 \pm 0.08954$ & 2024 & Nov & 29 & $03: 12: 54.0$ & $0.014 \pm 0.041$ & $18.61 \pm 1.73$ \\
\hline $5830.39948 \pm 0.05933$ & 2025 & Jun & 3 & $21: 35: 14.8$ & $0.079 \pm 0.044$ & $11.14 \pm 0.49$ \\
\hline $6016.99917 \pm 0.04807$ & 2025 & Dec & 7 & $11: 58: 48.0$ & $0.127 \pm 0.046$ & $8.58 \pm 0.24$ \\
\hline $6203.52776 \pm 0.04248$ & 2026 & Jun & 12 & $00: 39: 58.4$ & $0.165 \pm 0.047$ & $7.26 \pm 0.15$ \\
\hline $6390.01925 \pm 0.03950$ & 2026 & Dec & 15 & $12: 27: 43.1$ & $0.197 \pm 0.048$ & $6.46 \pm 0.11$ \\
\hline \multicolumn{7}{|l|}{ Outer Planet } \\
\hline$\overline{3575.38963} \pm 0.03743$ & 2019 & Apr & 1 & $21: 21: 04.0$ & $0.285 \pm 0.013$ & $05.78 \pm 0.04$ \\
\hline $3878.83019 \pm 0.11337$ & 2020 & Jan & 30 & $07: 55: 28.7$ & $0.213 \pm 0.018$ & $15.62 \pm 2.02$ \\
\hline $4179.70378 \pm 0.06532$ & 2020 & Nov & 26 & $04: 53: 26.9$ & $0.306 \pm 0.011$ & $06.10 \pm 0.07$ \\
\hline $4483.55306 \pm 0.05994$ & 2021 & Sep & 26 & $01: 16: 24.1$ & $0.148 \pm 0.014$ & $06.61 \pm 0.12$ \\
\hline $4784.28095 \pm 0.14007$ & 2022 & Jul & 23 & $18: 44: 34.3$ & $0.316 \pm 0.012$ & $12.09 \pm 1.76$ \\
\hline $5087.86638 \pm 0.07416$ & 2023 & May & 23 & $08: 47: 35.0$ & $0.156 \pm 0.014$ & $05.88 \pm 0.01$ \\
\hline $5391.64281 \pm 0.10567$ & 2024 & Mar & 22 & $03: 25: 38.7$ & $0.027 \pm 0.017$ & $08.53 \pm 0.44$ \\
\hline $5692.15323 \pm 0.09213$ & 2025 & Jan & 16 & $15: 40: 39.0$ & $0.175 \pm 0.013$ & $07.24 \pm 0.24$ \\
\hline $5996.00286 \pm 0.07245$ & 2025 & Nov & 16 & $12: 04: 06.7$ & $0.006 \pm 0.012$ & $06.10 \pm 0.09$ \\
\hline $6299.20901 \pm 0.26220$ & 2026 & Sep & 15 & $17: 00: 58.4$ & $0.038 \pm 0.040$ & $33.5 \pm 21.7$ \\
\hline
\end{tabular}

(This table is available in machine-readable form.)

the planets are now $<26 M_{\oplus}, \sim 7-43 M_{\oplus}$, and $\sim 2-5 M_{\oplus}$ for the inner, middle, and outer planets respectively. The radii are much better determined than the masses, and we find radii of 3.05, 7.0, and $4.7 R_{\oplus}$ for the inner, middle, and outer planets, respectively. The middle and outer planets have low bulk densities, $<0.68$ and $<0.26 \mathrm{~g} \mathrm{~cm}^{-3}$ at the $1 \sigma$ level. With such low densities, these two planets must have substantial hydrogen and helium atmospheres (Lopez \& Fortney 2014).

All of the orbits in the Kepler-47 system have eccentricities less than 0.07 at the $+1 \sigma$ level, and all four orbits have mutual inclinations aligned to within 1.6 of one another at the $+1 \sigma$ level. This nearly circular, co-planar, packed configuration is unlikely to have arisen as an outcome of strong gravitational scattering of the planets into their current orbits. Rather, the observations suggest that the planetary configuration is the result of relatively gentle migration in a circumbinary protoplanetary disk.

We found that the configuration is dynamically stable for at least 100 Myr. The new planet conforms with expectations based on an earlier analysis of a two-planet system (Hinse et al. 2015). As clearly demonstrated by the appearance and growth of the middle planet's transits, the planet's orbit precesses. The precession timescales for the inner, middle, and outer planets are calculated to be $\sim 10,245$, and $738 \mathrm{yr}$. The middle and outer planets are separated by $\sim 12$ mutual Hill radii, and we verify the earlier estimation (Kratter \& Shannon 2014) that they are dynamically packed. This is the first detection of a dynamically packed region in a circumbinary system, and it further confirms suspicions that planet formation and subsequent migration can proceed much like that around a single star, at least when far from the binary
(Pierens \& Nelson 2008, 2013; Kley \& Haghighipour 2014, 2015). We also find that, although they are close to having integer commensurate periods, the middle and outer planets are not in a mean-motion resonance - and yet they are gravitationally interacting and exchanging angular momentum, as indicated by their antiphased oscillations in inclination and eccentricity.

The average insolation (incident radiative flux) the outer planet receives from its two suns is $86.5 \%$ of the Sun-Earth value, placing it within the boundaries of the circumbinary habitable zone (Haghighipour \& Kaltenegger 2013). The middle planet's orbit straddles the "recent Venus" hot edge of the system's habitable zone. However, given their densities, neither planet is likely to host life.

Kepler was selected as the 10th mission of the Discovery Program. Funding for this mission is provided by the NASA Science Mission Directorate. The Kepler data were obtained from the Mikulski Archive for Space Telescopes (MAST). The Space Telescope Science Institute (STScI) is operated by the Association of Universities for Research in Astronomy, Inc., under NASA contract NAS5-26555. Support for MAST for non-HST data is provided by the NASA Office of Space Science via grant NXX09AF08G and by other grants and contracts. This work is based in part on observations obtained with the Hobby-Eberly Telescope, which is a joint project of the University of Texas at Austin, the Pennsylvania State University, Stanford University, Ludwig-Maximilians-Universität München, and Georg-August-Universität Göttingen. This research made use of data from the AAVSO Photometric AllSky Survey (APASS), funded by the Robert Martin Ayers 
Sciences Fund and NSF AST-1412587. This work presents results from the European Space Agency (ESA) space mission Gaia. Gaia data are being processed by the Gaia Data Processing and Analysis Consortium (DPAC). Funding for the DPAC is provided by national institutions, particularly the institutions participating in the Gaia MultiLateral Agreement (MLA). The Gaia mission website is https://www.cosmos. esa.int/gaia. The Gaia archive website is https://archives. esac.esa.int/gaia. Some of the simulations in this paper made use of the REBOUND code, which can be downloaded freely at http://github.com/hannorein/rebound. J.A.O. and W.F.W. acknowledge support from the Kepler Participating Scientist Program via NASA grant NNX14AB91G and support from NSF grant AST-1617004. J.A.O., W.F.W., G.W., and B.Q. also gratefully acknowledge support from NASA via grant NNX13AI76G. D.C.F. acknowledges support from the Alfred P. Sloan Research Fellowship. D.C.F. and S.M.M. were supported by NASA through the Kepler Participating Scientist Program award NNX14AB87G. N.H. acknowledges support from the NASA ADAP program under grant NNX13AF20G, NASA PAST program grant NNX14AJ38G, and NASA XRP program under grant $80 \mathrm{NSSC} 18 \mathrm{~K} 0519$. T.C.H. acknowledges KASI research grant \#2015-1-850-04. B.Q. gratefully acknowledges support by an appointment to the NASA Postdoctoral Program at the Ames Research Center, administered by Oak Ridge Associated Universities through a contract with NASA. G.T. acknowledges partial support for this work from NSF grant AST-1509375. E.B.F. acknowledges support from NASA Participating Scientists Program awards
NNX12AF73G and NNX14AN76G, NASA ADAP program under grant NNX13AF20G, as well as the University of Florida and the Pennsylvania State University's Center for Exoplanets and Habitable Worlds. The Center for Exoplanets and Habitable Worlds is supported by the Pennsylvania State University, the Eberly College of Science, and the Pennsylvania Space Grant Consortium. J.J.L.'s work on this project was supported by NASA's Astrophysics Data Analysis Program under grant no. 16-ADAP16-0034. Finally, we are deeply grateful to John Hood Jr. for his generous support of exoplanet research at San Diego State University.

\section{Appendix \\ Planetary Mass and Radius Data}

Table 11 lists the planetary masses, radii, and insolation values used in Figure 22. Note that the Marcy et al. (2014) sample includes many nondetections and upper limits that we do not plot.

We estimate the insolation each planet received from its host star using the cited $T_{\text {eff }}, R_{*}$ and $M_{*}$ of each host such that:

$$
\frac{S}{S_{\oplus}}=\left(\frac{T_{\mathrm{eff}}}{T_{\odot}}\right)^{4}\left(\frac{1 \mathrm{au}}{a}\right)^{2}\left(\frac{R_{\odot}}{R_{*}}\right)^{2}
$$

where the semimajor axis $a$ is calculated from the measured mass and orbital period (eccentricities are neglected). Uncertainties are added in quadrature.

Table 11

Planetary Mass and Radius Data

\begin{tabular}{|c|c|c|c|c|}
\hline Name & Mass $\left(M_{\oplus}\right)$ & Radius $\left(R_{\oplus}\right)$ & Insolation $\left(S_{\oplus}\right)$ & $\overline{\text { References }}$ \\
\hline $55 \mathrm{Cnc} \mathrm{e}$ & $7.81_{-0.53}^{+0.58}$ & $2.17_{-0.10}^{+0.10}$ & $2442.2_{-54.2}^{+54.2}$ & 13,39 \\
\hline GJ1214 b & $6.55_{-0.98}^{+0.98}$ & $2.68_{-0.13}^{+0.13}$ & $16.3_{-2.4}^{+2.4 .2}$ & 7 \\
\hline GJ3470 b & $14.00_{-1.80}^{+1.80}$ & $4.20_{-0.60}^{+0.60}$ & $29.7_{-6.8}^{+6.8}$ & 4 \\
\hline GJ436 b & $24.79_{-2.54}^{+2.22}$ & $4.05_{-0.17}^{+0.17}$ & $28.4_{-2.0}^{+3.0}$ & 36 \\
\hline GJ1132 b & $1.63_{-0.45}^{+0.54}$ & $1.44_{-0.21}^{+0.21}$ & $10.5_{-2.2}^{+2.2}$ & 40 \\
\hline GJ9827 K2-133 & $3.74_{-0.48}^{+0.50}$ & $1.62_{-0.16}^{+0.17}$ & $311.6_{-50.6}^{+50.6}$ & 41 \\
\hline GJ9827 K2-13 & $1.47_{-0.58}^{+0.59}$ & $1.27_{-0.13}^{+0.13}$ & $71.5_{-11.6}^{+11.6}$ & 41 \\
\hline GJ9827 K2-13 & $2.38_{-0.69}^{+0.71}$ & $2.09_{-0.21}^{+0.22}$ & $35.2_{-5.7}^{+5.7}$ & 41 \\
\hline CoRoT-7 b & $5.53_{-0.78}^{+0.86}$ & $1.58_{-0.10}^{+0.10}$ & $1572.7_{-121.9}^{+121.9}$ & 16 \\
\hline HAT-P-11 b & $26.70_{-2.22}^{+2.22}$ & $4.36_{-0.02}^{+0.10}$ & $83.5_{-2.1}^{+3.7}$ & 42 \\
\hline HAT-P-26 b & $18.75_{-2.22}^{+2.22}$ & $6.20_{-0.35}^{+0.79}$ & $162.9_{-13.8}^{+30.1}$ & 15 \\
\hline HD97658b & $7.55_{-0.79}^{+0.83}$ & $2.25_{-0.09}^{+0.10}$ & $54.4_{-3.9}^{+4.0}$ & 35 \\
\hline HD219134b & $4.46_{-0.47}^{+0.47}$ & $1.61_{-0.09}^{+0.09}$ & $181.0_{-4.3}^{+4.3}$ & 28 \\
\hline Kepler-4 b & $24.50_{-3.80}^{+3.80}$ & $4.50_{-0.12}^{+0.12}$ & $1239.5_{-44.0}^{+40.1}$ & 33 \\
\hline Kepler-10 b & $3.72_{-0.42}^{+0.42}$ & $1.47_{-0.02}^{+0.03}$ & $3574.9_{-227.0}^{+227.0}$ & 2 \\
\hline Kepler-10 c & $13.98_{-1.79}^{+1.79}$ & $2.35_{-0.04}^{+0.09}$ & $17.5_{-1.1}^{+1.1}$ & 10,38 \\
\hline Kepler-11 b & $1.90_{-1.00}^{+1.40}$ & $1.80_{-0.05}^{+0.03}$ & $125.3_{-5.4}^{+4.7}$ & 22 \\
\hline Kepler-11 c & $2.90_{-1.60}^{+2.90}$ & $2.87_{-0.06}^{+0.05}$ & $91.6_{-3.9}^{+3.5}$ & 22 \\
\hline Kepler-11 d & $7.30_{-1.50}^{+0.80}$ & $3.12_{-0.07}^{+0.06}$ & $43.7_{-1.9}^{+1.6}$ & 22 \\
\hline Kepler-11 e & $8.00_{-2.10}^{+1.50}$ & $4.19_{-0.09}^{+0.07}$ & $27.6_{-1.2}^{+1.0}$ & 22 \\
\hline Kepler-11 f & $2.00_{-0.90}^{+0.80}$ & $2.49_{-0.07}^{+0.04}$ & $16.69_{-0.72}^{+0.63}$ & 22 \\
\hline Kepler-18 b & $6.90_{-3.40}^{+3.40}$ & $2.00_{-0.10}^{+0.10}$ & $450.1_{-33.3}^{+33.3}$ & 8 \\
\hline Kepler-18 c & $17.30_{-1.90}^{+1.90}$ & $5.49_{-0.26}^{+0.26}$ & $160.1_{-11.9}^{+11.9}$ & 8 \\
\hline Kepler-18 d & $16.40_{-1.40}^{+1.40}$ & $6.98_{-0.33}^{+0.33}$ & $65.2_{-4.8}^{+4.8}$ & 8 \\
\hline Kepler-20 b & $8.70_{-2.20}^{+2.20}$ & $1.91_{-0.21}^{+0.12}$ & $346.3_{-51.8}^{+35.0}$ & 11,12 \\
\hline Kepler-20 c & $16.10_{-3.50}^{+3.50}$ & $3.07_{-0.31}^{+0.20}$ & $82.9_{-12.4}^{+8.4}$ & 11,12 \\
\hline Kepler-23 b & $1.30_{-0.50}^{+1.30}$ & $1.80_{-0.10}^{+0.10}$ & $450.8_{-36.3}^{+36.3}$ & 43 \\
\hline
\end{tabular}


Table 11

(Continued)

\begin{tabular}{|c|c|c|c|c|}
\hline Name & Mass $\left(M_{\oplus}\right)$ & Radius $\left(R_{\oplus}\right)$ & Insolation $\left(S_{\oplus}\right)$ & References \\
\hline Kepler-23 c & $2.20_{-0.90}^{+2.80}$ & $3.20_{-0.20}^{+0.20}$ & $259.9_{-21.0}^{+21.0}$ & 43 \\
\hline Kepler-24 b & $2.00_{-0.70}^{+1.60}$ & $2.70_{-0.50}^{+0.60}$ & $230.3_{-54.4}^{+124.1}$ & 43 \\
\hline Kepler-24 c & $1.70_{-0.70}^{+1.20}$ & $3.20_{-1.80}^{+1.70}$ & $132.4_{-31.3}^{+71.4}$ & 43 \\
\hline Kepler-25 b & $24.60_{-5.70}^{+5.70}$ & $5.20_{-0.09}^{+0.09}$ & $186.3_{-9.9}^{+9.9}$ & 25 \\
\hline Kepler-25 c & $9.60_{-4.20}^{+4.20}$ & $2.71_{-0.05}^{+0.05}$ & $481.7_{-25.5}^{+25.5}$ & 25 \\
\hline Kepler-26 b & $5.12_{-0.61}^{+0.65}$ & $2.78_{-0.11}^{+0.11}$ & $7.64_{-0.65}^{+0.65}$ & 20 \\
\hline Kepler-26 c & $6.20_{-0.65}^{+0.65}$ & $2.72_{-0.12}^{+0.12}$ & $4.85_{-0.41}^{+0.41}$ & 20 \\
\hline Kepler-27 b & $3.60_{-1.60}^{+1.70}$ & $4.90_{-0.30}^{+0.40}$ & $52.5_{-5.0}^{+3.6}$ & 43 \\
\hline Kepler-27 c & $3.60_{-1.60}^{+2.00}$ & $7.00_{-0.50}^{+0.50}$ & $20.2_{-1.9}^{+1.4}$ & 43 \\
\hline Kepler-28 b & $1.20_{-0.50}^{+0.80}$ & $1.80_{-0.10}^{+0.10}$ & $27.8_{-7.4}^{+7.4}$ & 43 \\
\hline Kepler-28 c & $1.00_{-0.30}^{+0.70}$ & $1.70_{-0.10}^{+0.20}$ & $15.9_{-4.2}^{+4.2}$ & 43 \\
\hline Kepler-29 b & $4.51_{-1.47}^{+1.41}$ & $3.35_{-0.22}^{+0.22}$ & $96.8_{-10.4}^{+10.4}$ & 20 \\
\hline Kepler-29 c & $4.00_{-1.29}^{+1.23}$ & $3.14_{-0.20}^{+0.20}$ & $69.3_{-7.4}^{+7.4}$ & 20 \\
\hline Kepler-30 b & $11.30_{-1.40}^{+1.40}$ & $3.90_{-0.20}^{+0.20}$ & $21.5_{-4.1}^{+4.1}$ & 31 \\
\hline Kepler-30 d & $23.10_{-2.70}^{+2.70}$ & $8.80_{-0.50}^{+0.50}$ & $2.59_{-0.50}^{+0.50}$ & 31 \\
\hline Kepler-31 b & $0.70_{-0.60}^{+2.40}$ & $5.60_{-1.00}^{+1.00}$ & $92.7_{-41.4}^{+44.8}$ & 43 \\
\hline Kepler-31 c & $2.20_{-1.20}^{+4.60}$ & $5.30_{-1.00}^{+0.90}$ & $35.8_{-16.0}^{+17.3}$ & 43 \\
\hline Kepler-31 d & $2.80_{-1.40}^{+4.20}$ & $4.20_{-0.80}^{+0.80}$ & $13.7_{-6.1}^{+6.6}$ & 43 \\
\hline Kepler-32 b & $0.50_{-0.10}^{+0.30}$ & $2.00_{-0.10}^{+0.20}$ & $16.8_{-2.8}^{+2.1}$ & 43 \\
\hline Kepler-32 c & $0.40_{-0.10}^{+0.20}$ & $1.90_{-0.20}^{+0.20}$ & $9.9_{-1.6}^{+1.2}$ & 43 \\
\hline Kepler-33 c & $0.80_{-0.70}^{+2.50}$ & $3.20_{-0.30}^{+0.30}$ & $255.5_{-38.3}^{+31.1}$ & 14,22 \\
\hline Kepler-33 d & $4.70_{-2.00}^{+2.00}$ & $5.40_{-0.50}^{+0.50}$ & $130.8_{-19.6}^{+15.9}$ & 14,22 \\
\hline Kepler-33 e & $6.70_{-1.30}^{+1.20}$ & $4.00_{-0.40}^{+0.40}$ & $79.0_{-11.8}^{+9.6}$ & 14,22 \\
\hline Kepler-33 f & $11.50_{-2.10}^{+1.80}$ & $4.50_{-0.40}^{+0.40}$ & $56.2_{-8.4}^{+6.8}$ & 14,22 \\
\hline Kepler-36 b & $4.45_{-0.27}^{+0.33}$ & $1.49_{-0.04}^{+0.03}$ & $217.4_{-9.3}^{+9.3}$ & 6 \\
\hline Kepler-36 c & $8.08_{-0.46}^{+0.60}$ & $3.68_{-0.05}^{+0.05}$ & $175.8_{-7.6}^{+7.6}$ & 6 \\
\hline Kepler-47 b & $2.07_{-2.07}^{+23.70}$ & $3.05_{-0.04}^{+0.04}$ & $11.74_{-0.43}^{+0.43}$ & This work \\
\hline Kepler-47 c & $19.02_{-11.67}^{+23.84}$ & $7.04_{-0.49}^{+0.66}$ & $1.989_{-0.072}^{+0.072}$ & This work \\
\hline Kepler-47 d & $3.17_{-1.25}^{+2.18}$ & $4.65_{-0.07}^{+0.09}$ & $1.047_{-0.038}^{+0.038}$ & This work \\
\hline Kepler-48 b & $1.90_{-1.29}^{+5.34}$ & $1.89_{-0.23}^{+0.23}$ & $171.4_{-31.0}^{+31.0}$ & 25 \\
\hline Kepler-48 c & $2.81_{-1.58}^{+3.78}$ & $2.61_{-0.32}^{+0.32}$ & $66.9_{-12.1}^{+12.1}$ & 25 \\
\hline Kepler-49 b & $5.09_{-1.94}^{+2.11}$ & $2.35_{-0.09}^{+0.09}$ & $14.7_{-2.0}^{+2.0}$ & 20 \\
\hline Kepler-49 c & $3.28_{-1.32}^{+1.45}$ & $2.06_{-0.09}^{+0.09}$ & $8.5_{-1.2}^{+1.2}$ & 20 \\
\hline Kepler-51 b & $2.10_{-0.80}^{+1.50}$ & $7.10_{-0.30}^{+0.30}$ & $16.5_{-2.1}^{+2.1}$ & 26 \\
\hline Kepler-51 c & $4.00_{-0.40}^{+0.40}$ & $9.00_{-1.70}^{+2.80}$ & $7.04_{-0.88}^{+0.88}$ & 26 \\
\hline Kepler-51 d & $7.60_{-1.10}^{+1.10}$ & $9.70_{-0.50}^{+0.50}$ & $4.01_{-0.50}^{+0.50}$ & 26 \\
\hline Kepler-52 b & $2.30_{-0.80}^{+1.50}$ & $2.30_{-0.10}^{+0.20}$ & $23.0_{-2.2}^{+1.9}$ & 43 \\
\hline Kepler-52 c & $6.30_{-2.00}^{+5.00}$ & $2.10_{-0.10}^{+0.10}$ & $8.67_{-0.82}^{+0.73}$ & 43 \\
\hline Kepler-53 c & $2.80_{-0.90}^{+1.90}$ & $3.40_{-0.40}^{+0.40}$ & $57.0_{-31.8}^{+31.8}$ & 43 \\
\hline Kepler-53 d & $3.50_{-1.10}^{+2.30}$ & $3.60_{-0.40}^{+0.50}$ & $21.7_{-12.1}^{+12.1}$ & 43 \\
\hline Kepler-54 b & $0.80_{-0.30}^{+1.00}$ & $2.30_{-0.30}^{+0.40}$ & $10.0_{-2.1}^{+2.1}$ & 43 \\
\hline Kepler-54 c & $0.70_{-0.30}^{+0.90}$ & $1.30_{-0.10}^{+0.10}$ & $5.8_{-1.2}^{+1.2}$ & 43 \\
\hline Kepler-55 b & $1.30_{-0.60}^{+2.10}$ & $1.90_{-0.10}^{+0.20}$ & $5.99_{-0.45}^{+0.65}$ & 43 \\
\hline Kepler-55 c & $1.80_{-0.80}^{+2.80}$ & $2.50_{-0.30}^{+0.30}$ & $3.47_{-0.26}^{+0.38}$ & 43 \\
\hline Kepler-57 b & $16.85_{-6.95}^{+7.24}$ & $1.88_{-0.14}^{+0.14}$ & $73.7_{-9.0}^{+9.0}$ & 20 \\
\hline Kepler-57 c & $4.12_{-1.89}^{+1.97}$ & $1.39_{-0.10}^{+0.10}$ & $28.9_{-3.5}^{+3.5}$ & 20 \\
\hline Kepler-58 b & $2.90_{-1.10}^{+2.10}$ & $2.80_{-0.60}^{+0.50}$ & $171.6_{-38.3}^{+84.0}$ & 43 \\
\hline Kepler-58 c & $2.30_{-0.90}^{+1.80}$ & $2.70_{-0.50}^{+0.60}$ & $97.8_{-21.9}^{+47.9}$ & 43 \\
\hline Kepler-60 b & $4.18_{-0.52}^{+0.56}$ & $1.71_{-0.13}^{+0.13}$ & $319.1_{-41.9}^{+41.9}$ & 20 \\
\hline Kepler-60 c & $3.85_{-0.81}^{+0.81}$ & $1.90_{-0.15}^{+0.15}$ & $236.9_{-31.1}^{+31.1}$ & 20 \\
\hline Kepler-60 d & $4.16_{-0.52}^{+0.56}$ & $1.99_{-0.16}^{+0.16}$ & $161.3_{-21.2}^{+21.2}$ & 20 \\
\hline Kepler-68 b & $5.97_{-1.70}^{+1.70}$ & $2.33_{-0.02}^{+0.02}$ & $406.9_{-20.8}^{+20.8}$ & 25 \\
\hline Kepler-78 b & $1.69_{-0.41}^{+0.41}$ & $1.20_{-0.09}^{+0.09}$ & $3973.8_{-432.5}^{+432.5}$ & 17,30 \\
\hline Kepler-79 b & $10.90_{-6.00}^{+7.40}$ & $3.47_{-0.07}^{+0.07}$ & $162.5_{-8.8}^{+8.8}$ & 18 \\
\hline Kepler-79 c & $5.90_{-2.30}^{+1.90}$ & $3.72_{-0.08}^{+0.08}$ & $63.1_{-3.4}^{+3.4}$ & 18 \\
\hline Kepler-79 d & $6.00_{-1.60}^{+2.10}$ & $7.16_{-0.16}^{+0.13}$ & $26.8_{-1.4}^{+1.4}$ & 18 \\
\hline Kepler-79 e & $4.10_{-1.10}^{+1.20}$ & $3.49_{-0.14}^{+0.14}$ & $14.85_{-0.80}^{+0.80}$ & 18 \\
\hline Kepler-80 d & $6.75_{-0.51}^{+0.69}$ & $1.53_{-0.07}^{+0.09}$ & $126.3_{-8.8}^{+8.8}$ & 24 \\
\hline
\end{tabular}


Table 11

(Continued)

\begin{tabular}{|c|c|c|c|c|}
\hline Name & Mass $\left(M_{\oplus}\right)$ & Radius $\left(R_{\oplus}\right)$ & Insolation $\left(S_{\oplus}\right)$ & References \\
\hline Kepler-80 e & $4.92_{-0.37}^{+0.49}$ & $1.60_{-0.07}^{+0.08}$ & $72.8_{-5.1}^{+5.1}$ & 24 \\
\hline Kepler-80 b & $5.99_{-0.57}^{+0.49}$ & $2.67_{-0.10}^{+0.10}$ & $41.7_{-2.9}^{+2.9}$ & 24 \\
\hline Kepler-80 c & $5.03_{-0.42}^{+0.40}$ & $2.74_{-0.10}^{+0.12}$ & $28.0_{-2.0}^{+2.0}$ & 24 \\
\hline Kepler-81 b & $0.20_{-0.10}^{+1.70}$ & $2.40_{-0.10}^{+0.10}$ & $40.3_{-3.5}^{+3.6}$ & 43 \\
\hline Kepler-81 c & $0.10_{-0.10}^{+1.10}$ & $2.30_{-0.10}^{+0.10}$ & $15.8_{-1.4}^{+1.4}$ & 43 \\
\hline Kepler-84 b & $5.00_{-2.90}^{+5.70}$ & $2.50_{-0.40}^{+0.40}$ & $221.4_{-48.3}^{+52.7}$ & 43 \\
\hline Kepler-85 b & $0.70_{-0.40}^{+1.90}$ & $1.80_{-0.20}^{+0.20}$ & $99.4_{-12.5}^{+17.5}$ & 43 \\
\hline Kepler-85 c & $1.00_{-0.60}^{+2.20}$ & $1.90_{-0.20}^{+0.20}$ & $57.5_{-7.2}^{+10.1}$ & 43 \\
\hline Kepler-85 e & $0.60_{-0.40}^{+0.50}$ & $1.20_{-0.10}^{+0.10}$ & $22.6_{-2.8}^{+4.0}$ & 43 \\
\hline Kepler-87 c & $6.40_{-0.80}^{+0.80}$ & $6.14_{-0.29}^{+0.29}$ & $6.51_{-0.34}^{+0.34}$ & 29 \\
\hline Kepler-89 b & $10.50_{-4.60}^{+4.60}$ & $1.71_{-0.16}^{+0.16}$ & $1194.7_{-161.8}^{+161.8}$ & 37 \\
\hline Kepler-89 c & $9.40_{-2.10}^{+2.40}$ & $4.32_{-0.41}^{+0.41}$ & $295.9_{-40.1}^{+40.1}$ & 26,37 \\
\hline Kepler-89 e & $13.00_{-2.10}^{+2.50}$ & $6.56_{-0.62}^{+0.62}$ & $32.8_{-4.4}^{+4.4}$ & 26,37 \\
\hline Kepler-93 b & $4.02_{-0.48}^{+0.68}$ & $1.48_{-0.02}^{+0.02}$ & $274.1_{-11.8}^{+11.8}$ & 9 \\
\hline Kepler-94 b & $10.84_{-1.40}^{+1.40}$ & $3.51_{-0.15}^{+0.15}$ & $238.7_{-22.0}^{+22.0}$ & 25 \\
\hline Kepler-95 b & $13.00_{-2.90}^{+2.90}$ & $3.42_{-0.09}^{+0.09}$ & $179.3_{-13.8}^{+13.8}$ & 25 \\
\hline Kepler-98 b & $3.55_{-1.60}^{+1.60}$ & $1.99_{-0.22}^{+0.22}$ & $1530.0_{-249.1}^{+249.1}$ & 25 \\
\hline Kepler-99 b & $6.15_{-1.30}^{+1.30}$ & $1.48_{-0.08}^{+0.08}$ & $99.7_{-11.3}^{+11.3}$ & 25 \\
\hline Kepler-102 e & $8.93_{-2.00}^{+2.00}$ & $2.22_{-0.07}^{+0.07}$ & $21.1_{-1.6}^{+1.6}$ & 25 \\
\hline KOI-115.03 & $1.13_{-0.83}^{+1.21}$ & $0.73_{-0.04}^{+0.04}$ & $423.0_{-36.4}^{+36.4}$ & 20 \\
\hline Kepler-105 b & $3.74_{-1.99}^{+1.99}$ & $2.22_{-0.11}^{+0.11}$ & $233.3_{-20.1}^{+20.1}$ & 20 \\
\hline Kepler-105 c & $4.60_{-0.88}^{+0.92}$ & $1.31_{-0.07}^{+0.07}$ & $161.6_{-13.9}^{+13.9}$ & 20 \\
\hline Kepler-106 c & $10.44_{-3.20}^{+3.20}$ & $2.50_{-0.32}^{+0.32}$ & $92.2_{-22.1}^{+22.1}$ & 25 \\
\hline Kepler-114 c & $0.80_{-0.30}^{+0.60}$ & $1.00_{-0.10}^{+0.10}$ & $15.0_{-1.6}^{+1.5}$ & 43 \\
\hline Kepler-122 f & $2.10_{-1.00}^{+2.50}$ & $2.30_{-0.40}^{+0.30}$ & $20.5_{-5.0}^{+5.0}$ & 43 \\
\hline Kepler-127 b & $0.80_{-0.40}^{+2.30}$ & $1.40_{-0.04}^{+0.10}$ & $146.9_{-7.7}^{+12.9}$ & 43 \\
\hline Kepler-127 c & $1.00_{-0.60}^{+3.50}$ & $2.40_{-0.10}^{+0.10}$ & $56.9_{-3.0}^{+5.0}$ & 43 \\
\hline Kepler-127 d & $6.10_{-3.50}^{+3.10}$ & $2.60_{-0.10}^{+0.10}$ & $29.1_{-1.5}^{+2.5}$ & 43 \\
\hline Kepler-128 b & $1.15_{-0.52}^{+1.92}$ & $1.43_{-0.03}^{+0.03}$ & $210.0_{-13.7}^{+13.7}$ & 44 \\
\hline Kepler-128 c & $1.30_{-0.59}^{+2.25}$ & $1.42_{-0.03}^{+0.03}$ & $121.1_{-7.9}^{+7.9}$ & 44 \\
\hline Kepler-131 b & $16.13_{-3.50}^{+3.50}$ & $2.41_{-0.20}^{+0.20}$ & $63.1_{-9.3}^{+9.3}$ & 25 \\
\hline Kepler-138 b & $0.07_{-0.04}^{+0.06}$ & $0.52_{-0.03}^{+0.03}$ & $11.2_{-1.6}^{+1.6}$ & 19 \\
\hline Kepler-138 c & $1.97_{-1.12}^{+1.91}$ & $1.20_{-0.07}^{+0.07}$ & $7.6_{-1.1}^{+1.1}$ & 19 \\
\hline Kepler-138 d & $0.64_{-0.39}^{+0.67}$ & $1.21_{-0.08}^{+0.08}$ & $3.82_{-0.56}^{+0.56}$ & 19 \\
\hline Kepler-176 c & $0.10_{-0.04}^{+0.30}$ & $2.50_{-0.20}^{+0.10}$ & $37.7_{-4.2}^{+4.5}$ & 43 \\
\hline Kepler-176 d & $0.20_{-0.10}^{+0.50}$ & $2.30_{-0.20}^{+0.10}$ & $14.8_{-1.6}^{+1.8}$ & 43 \\
\hline Kepler-223 b & $7.40_{-1.10}^{+1.30}$ & $2.99_{-0.27}^{+0.18}$ & $653.7_{-91.4}^{+70.0}$ & 27 \\
\hline Kepler-223 c & $5.10_{-1.10}^{+1.70}$ & $3.44_{-0.30}^{+0.20}$ & $445.4_{-62.3}^{+47.7}$ & 27 \\
\hline Kepler-223 d & $8.00_{-1.30}^{+1.50}$ & $5.24_{-0.45}^{+0.26}$ & $258.9_{-36.2}^{+27.7}$ & 27 \\
\hline Kepler-223 e & $4.80_{-1.20}^{+1.40}$ & $4.60_{-0.41}^{+0.27}$ & $176.4_{-24.7}^{+18.9}$ & 27 \\
\hline Kepler-231 b & $4.90_{-1.30}^{+1.80}$ & $1.82_{-0.25}^{+0.26}$ & $7.6_{-1.5}^{+1.6}$ & 21 \\
\hline Kepler-231 c & $2.20_{-1.10}^{+1.50}$ & $1.69_{-0.23}^{+0.24}$ & $3.35_{-0.68}^{+0.72}$ & 21 \\
\hline Kepler-238 f & $4.50_{-2.20}^{+5.40}$ & $2.80_{-0.50}^{+0.50}$ & $24.5_{-7.0}^{+6.4}$ & 43 \\
\hline Kepler-277 b & $3.50_{-1.60}^{+2.80}$ & $2.90_{-0.70}^{+0.50}$ & $202.4_{-71.4}^{+43.7}$ & 43 \\
\hline Kepler-277 c & $4.50_{-2.00}^{+3.70}$ & $3.00_{-0.70}^{+0.50}$ & $85.7_{-30.2}^{+18.5}$ & 43 \\
\hline Kepler-279 c & $7.40_{-1.50}^{+2.20}$ & $5.50_{-1.00}^{+1.10}$ & $24.34_{-13.21}^{+0.84}$ & 43 \\
\hline Kepler-279 d & $4.50_{-0.90}^{+1.20}$ & $4.30_{-0.90}^{+1.00}$ & $13.89_{-7.54}^{+0.48}$ & 43 \\
\hline Kepler-289 b & $7.30_{-6.80}^{+6.80}$ & $2.15_{-0.10}^{+0.10}$ & $25.47_{-0.88}^{+0.88}$ & 32 \\
\hline Kepler-289 c & $4.00_{-0.90}^{+0.90}$ & $2.68_{-0.17}^{+0.17}$ & $10.73_{-0.37}^{+0.37}$ & 32 \\
\hline Kepler-305 b & $2.70_{-1.10}^{+2.90}$ & $3.10_{-0.20}^{+0.20}$ & $118.0_{-11.2}^{+12.7}$ & 43 \\
\hline Kepler-305 c & $1.40_{-0.70}^{+1.20}$ & $2.70_{-0.20}^{+0.20}$ & $68.1_{-6.5}^{+7.3}$ & 43 \\
\hline Kepler-305 d & $9.10_{-3.80}^{+6.10}$ & $2.70_{-0.20}^{+0.20}$ & $26.7_{-2.5}^{+2.9}$ & 43 \\
\hline Kepler-307 b & $7.44_{-0.87}^{+0.91}$ & $2.35_{-0.09}^{+0.09}$ & $60.4_{-3.8}^{+3.8}$ & 20 \\
\hline Kepler-307 c & $3.64_{-0.58}^{+0.65}$ & $2.20_{-0.07}^{+0.07}$ & $44.6_{-2.8}^{+2.8}$ & 20 \\
\hline Kepler-310 d & $7.00_{-4.10}^{+3.40}$ & $2.10_{-0.10}^{+0.20}$ & $3.58_{-0.38}^{+0.41}$ & 43 \\
\hline KOI1831.03 & $1.00_{-0.40}^{+1.20}$ & $1.20_{-0.10}^{+0.10}$ & $12.1_{-1.2}^{+1.5}$ & 43 \\
\hline Kepler-345 b & $0.50_{-0.30}^{+0.30}$ & $0.80_{-0.10}^{+0.10}$ & $35.2_{-2.7}^{+4.0}$ & 43 \\
\hline Kepler-345 c & $2.20_{-0.90}^{+0.90}$ & $1.30_{-0.10}^{+0.10}$ & $25.7_{-1.9}^{+2.9}$ & 43 \\
\hline
\end{tabular}


Table 11

(Continued)

\begin{tabular}{|c|c|c|c|c|}
\hline Name & Mass $\left(M_{\oplus}\right)$ & Radius $\left(R_{\oplus}\right)$ & Insolation $\left(S_{\oplus}\right)$ & References \\
\hline Kepler-359 c & $2.90_{-1.90}^{+2.40}$ & $4.80_{-0.90}^{+1.00}$ & $16.3_{-3.5}^{+7.4}$ & 43 \\
\hline Kepler-359 d & $2.70_{-1.50}^{+2.50}$ & $4.60_{-0.90}^{+0.90}$ & $11.1_{-2.4}^{+5.0}$ & 43 \\
\hline Kepler-396 b & $1.10_{-0.20}^{+0.50}$ & $3.30_{-0.40}^{+0.30}$ & $14.6_{-2.2}^{+3.0}$ & 43 \\
\hline Kepler-396 c & $1.10_{-0.20}^{+0.60}$ & $5.60_{-0.60}^{+0.50}$ & $5.6_{-0.8}^{+1.2}$ & 43 \\
\hline Kepler-406 b & $6.35_{-1.40}^{+1.40}$ & $1.43_{-0.03}^{+0.03}$ & $739.6_{-43.9}^{+43.9}$ & 25 \\
\hline Kepler-444 d & $0.04_{-0.02}^{+0.06}$ & $0.54_{-0.02}^{+0.02}$ & $89.2_{-7.9}^{+8.0}$ & 45 \\
\hline Kepler-444 e & $0.03_{-0.02}^{+0.06}$ & $0.56_{-0.02}^{+0.02}$ & $66.2_{-5.8}^{+5.9}$ & 45 \\
\hline Kepler-526 c & $0.50_{-0.30}^{+0.40}$ & $0.80_{-0.10}^{+0.10}$ & $250.4_{-31.0}^{+57.9}$ & 43 \\
\hline Kepler-549 b & $11.00_{-3.20}^{+4.20}$ & $3.10_{-0.40}^{+0.40}$ & $12.9_{-2.2}^{+2.3}$ & 43 \\
\hline $\mathrm{K} 2-2 \mathrm{~b}$ & $11.82_{-1.33}^{+1.33}$ & $2.53_{-0.18}^{+0.18}$ & $50.1_{-2.9}^{+2.9}$ & 46 \\
\hline $\mathrm{K} 2-3 \mathrm{~b}$ & $6.60_{-1.10}^{+1.10}$ & $2.29_{-0.23}^{+0.23}$ & $11.6_{-1.9}^{+1.9}$ & 47 \\
\hline $\mathrm{K} 2-3 \mathrm{c}$ & $3.10_{-1.20}^{+1.30}$ & $1.77_{-0.18}^{+0.18}$ & $3.50_{-0.58}^{+0.58}$ & 47 \\
\hline $\mathrm{K} 2-3 \mathrm{~d}$ & $1.20_{-0.70}^{+1.40}$ & $1.65_{-0.17}^{+0.17}$ & $1.59_{-0.26}^{+0.26}$ & 47 \\
\hline $\mathrm{K} 2-18 \mathrm{~b}$ & $7.96_{-1.91}^{+1.91}$ & $2.24_{-0.23}^{+0.23}$ & $1.06_{-0.18}^{+0.18}$ & 48 \\
\hline K2-19 c & $15.90_{-2.80}^{+7.70}$ & $4.51_{-0.47}^{+0.47}$ & $62.7_{-10.9}^{+10.9}$ & 49 \\
\hline $\mathrm{K} 2-38 \mathrm{~b}$ & $12.00_{-2.90}^{+2.90}$ & $1.55_{-0.16}^{+0.16}$ & $466.2_{-57.6}^{+57.6}$ & 50 \\
\hline $\mathrm{K} 2-38 \mathrm{c}$ & $9.90_{-4.60}^{+4.60}$ & $2.42_{-0.29}^{+0.29}$ & $128.4_{-15.9}^{+15.9}$ & 50 \\
\hline $\mathrm{K} 2-66 \mathrm{~b}$ & $21.30_{-3.60}^{+3.60}$ & $2.49_{-0.34}^{+0.34}$ & $840.4_{-89.9}^{+89.9}$ & 51 \\
\hline $\mathrm{K} 2-110 \mathrm{~b}$ & $16.70_{-3.20}^{+3.20}$ & $2.60_{-0.10}^{+0.10}$ & $27.6_{-1.3}^{+1.3}$ & 52 \\
\hline $\mathrm{K} 2-106 \mathrm{~b}$ & $8.36_{-0.94}^{+0.96}$ & $1.52_{-0.16}^{+0.16}$ & $3469.2_{-535.4}^{+535.4}$ & 51 \\
\hline K2-106 c & $5.80_{-3.00}^{+3.30}$ & $2.50_{-0.26}^{+0.27}$ & $52.0_{-8.0}^{+8.0}$ & 51 \\
\hline $\mathrm{K} 2-131 \mathrm{~b}$ & $6.80_{-1.60}^{+1.60}$ & $1.81_{-0.12}^{+0.16}$ & $4763.9_{-339.3}^{+339.3}$ & 53 \\
\hline $\mathrm{K} 2-111 \mathrm{~b}$ & $8.60_{-3.90}^{+3.90}$ & $1.90_{-0.20}^{+0.20}$ & $528.8_{-81.2}^{+81.2}$ & 54 \\
\hline $\mathrm{K} 2-106 \mathrm{~b}$ & $9.00_{-1.60}^{+1.60}$ & $1.82_{-0.14}^{+0.20}$ & $4301.3_{-347.6}^{+347.6}$ & 51 \\
\hline LHS1140 b & $6.65_{-1.82}^{+1.82}$ & $1.43_{-0.10}^{+0.10}$ & $0.390_{-0.062}^{+0.062}$ & 54 \\
\hline Trappist1 b & $1.02_{-0.15}^{+0.14}$ & $1.12_{-0.03}^{+0.03}$ & $4.26_{-0.40}^{+0.40}$ & 56 \\
\hline Trappist1 c & $1.16_{-0.14}^{+0.13}$ & $1.10_{-0.03}^{+0.03}$ & $2.27_{-0.22}^{+0.22}$ & 56 \\
\hline Trappist1 d & $0.30_{-0.04}^{+0.04}$ & $0.78_{-0.02}^{+0.02}$ & $1.15_{-0.11}^{+0.11}$ & 56 \\
\hline Trappist1 e & $0.77_{-0.08}^{+0.08}$ & $0.91_{-0.03}^{+0.03}$ & $0.663_{-0.063}^{+0.063}$ & 56 \\
\hline Trappist1 f & $0.93_{-0.08}^{+0.08}$ & $1.05_{-0.03}^{+0.03}$ & $0.383_{-0.036}^{+0.036}$ & 56 \\
\hline Trappist1 g & $0.33_{-0.06}^{+0.05}$ & $0.77_{-0.03}^{+0.03}$ & $0.383_{-0.036}^{+0.036}$ & 56 \\
\hline Wasp-47 d & $15.20_{-7.00}^{+7.00}$ & $3.63_{-0.14}^{+0.14}$ & $155.1_{-12.9}^{+12.9}$ & 3 \\
\hline $\mathrm{K} 2-229 \mathrm{~b}$ & $2.59_{-0.430}^{+0.430}$ & $1.164_{-0.048}^{+0.066}$ & $2454.3_{-110.3}^{+150.4}$ & 57 \\
\hline
\end{tabular}

References. (1) Almenara et al. (2015); (2) Batalha et al. (2011); (3) Becker et al. (2015); (4) Bonfils et al. (2012); (5) Borucki et al. (2010a); (6) Carter et al. (2012); (7) Charbonneau et al. (2009); (8) Cochran et al. (2011); (9) Dressing et al. (2015); (10) Dumusque et al. (2014); (11) Fressin et al. (2012); (12) Gautier et al. (2012); (13) Gillon et al. (2012); (14) Hadden \& Lithwick (2016); (15) Hartman et al. (2011); (16) Haywood et al. (2014); (17) Howard et al. (2013); (18) Jontof-Hutter et al. (2014); (19) Jontof-Hutter et al. (2015); (20) Jontof-Hutter et al. (2016); (21) Kipping et al. (2014); (22) Lissauer et al. (2012); (23) Lissauer et al. (2013); (24) MacDonald et al. (2016); (25) Marcy et al. (2014); (26) Masuda (2014); (27) Mills et al. (2016); (28) Motalebi et al. (2015); (29) Ofir et al. (2014); (30) Pepe et al. (2013); (31) Sanchis-Ojeda et al. (2012); (32) Schmitt et al. (2014); (33) Silva Aguirre et al. (2015); (34) Vanderburg et al. (2015); (35) van Grootel et al. (2014); (36) von Braun et al. (2012); (37) Weiss et al. (2013); (38) Weiss et al. (2016); (39) Winn et al. (2011); (40) Southworth et al. (2017); (41) Prieto-Arranz et al. (2018); (42) Southworth (2011); (43) Hadden \& Lithwick (2017); (44) Hadden \& Lithwick (2016); (45) Mills \& Fabrycky (2017); (46) Vanderburg et al. (2015); (47) Damasso et al. (2018); (48) Cloutier et al. (2017); (49) Narita et al. (2015); (50) Sinukoff et al. (2016); (51) Sinukoff et al. (2017); (52) Osborn et al. (2017); (53) Dai et al. (2017); (54) Fridlund et al. (2017); (55) Dittmann et al. (2017); (56) Grimm et al. (2018); (57) Santerne et al. (2018).

(This table is available in machine-readable form.)

\section{ORCID iDs}

Jerome A. Orosz (iD https://orcid.org/0000-0001-9647-2886 William F. Welsh (iD https://orcid.org/0000-0003-2381-5301 Nader Haghighipour (1D https://orcid.org/0000-0002-5234-6375 Sean M. Mills (iD https://orcid.org/0000-0002-4535-6241 Guillermo Torres (iD https://orcid.org/0000-0002-5286-0251 Eric Agol (i) https://orcid.org/0000-0002-0802-9145 Daniel C. Fabrycky (i) https://orcid.org/0000-0003-3750-0183 Daniel Jontof-Hutter (iD https://orcid.org/0000-0002-6227-7510 Tobias C. Hinse (iD https://orcid.org/0000-0001-8870-3146 William D. Cochran (iD https://orcid.org/0000-0001-9662-3496
Michael Endl (1D https://orcid.org/0000-0002-7714-6310 Eric B. Ford (iD https://orcid.org/0000-0001-6545-639X

\section{References}

Agol, E., Steffen, J., Sari, R., \& Clarkson, W. 2005, MNRAS, 359, 567 Allard, F., Hauschildt, P. H., Alexander, D. R., \& Starrfield, S. 1997, ARA\&A, 35,137

Almenara, J. M., Astudillo-Defru, N., Bonfils, X., et al. 2015, A\&A, 581, L7 Bailer-Jones, C. A. L., Rybizki, J., Fouesneau, M., Mantelet, G., \& Andrae, R. 2018, AJ, 156, 58

Bass, G. P., Orosz, J. A., Welsh, W. F., et al. 2012, ApJ, 761, 157

Batalha, N. M., Borucki, W. J., Bryson, S. T., et al. 2011, ApJ, 729, 27 
Becker, J. C., Vanderburg, A., Adams, F. C., et al. 2015, ApJL, 812, L18 Bevington, P. R. 1969, Data Reduction and Error Analysis for the Physical Sciences (New York: McGraw-Hill)

Bonfils, X., Gillon, M., Udry, S., et al. 2012, A\&A, 546, A27

Borucki, W. J., Koch, D. G., Basri, G., et al. 2010a, Sci, 327, 977

Borucki, W. J., Koch, D. G., Brown, T. M., et al. 2010b, ApJL, 713, L126

Carter, J. A., Agol, E., Chaplin, W. J., et al. 2012, Sci, 337, 556

Carter, J. A., Fabrycky, D. C., \& Ragozzine, D. 2011, Sci, 331, 562

Chambers, J. E., Quintana, E. V., Duncan, M. J., \& Lissauer, J. J. 2002, AJ, 123,2884

Chambers, J. E., Wetherill, G. W., \& Boss, A. P. 1996, Icar, 119, 261

Charbonneau, D., Berta, Z. K., Irwin, J., et al. 2009, Natur, 462, 891

Charbonneau, P. 1995, ApJS, 101, 309

Cincotta, P. M., Giordano, C. M., \& Simó, C. 2003, PhyD, 182, 151

Clemence, G. M. 1965, ARA\&A, 3, 93

Cloutier, R., Astudillo-Defru, N., Doyon, R., et al. 2017, A\&A, 608, A35

Cochran, W. D., Fabrycky, D. C., Torres, G., et al. 2011, ApJS, 197, 7

Dai, F., Winn, J. N., Gandolfi, D., et al. 2017, AJ, 154, 226

Damasso, M., Bonomo, A. S., Astudillo-Defru, N., et al. 2018, A\&A, 615, A69

de Pater, I., \& Lissauer, J. J. 2015, Planetary Sciences (Cambridge: Cambridge Univ. Press)

Dittmann, J. A., Irwin, J. M., Charbonneau, D., et al. 2017, Natur, 544, 333

Dotter, A., Chaboyer, B., Jevremović, D., et al. 2008, ApJS, 178, 89

Doyle, L. R., Carter, J. A., Fabrycky, D. C., et al. 2011, Sci, 333, 1602

Dressing, C. D., Charbonneau, D., Dumusque, X., et al. 2015, ApJ, 800, 135

Dumusque, X., Bonomo, A. S., Haywood, R. D., et al. 2014, ApJ, 789, 154

Dunhill, A. C., \& Alexander, R. D. 2013, MNRAS, 435, 2328

Dvorak, R. 1982, OAWMN, 191, 423

Eggleton, P. P., Kiseleva, L. G., \& Hut, P. 1998, ApJ, 499, 853

Flower, P. J. 1996, ApJ, 469, 355

Fressin, F., Torres, G., Rowe, J. F., et al. 2012, Natur, 482, 195

Fridlund, M., Gaidos, E., Barragán, O., et al. 2017, A\&A, 604, A16

Gaia Collaboration, Brown, A. G. A., Vallenari, A., et al. 2018, A\&A, 616, A1

Gaia Collaboration, Prusti, T., de Bruijne, J. H. J., et al. 2016, A\&A, 616, A1

Gautier, T. N., III, Charbonneau, D., Rowe, J. F., et al. 2012, ApJ, 749, 15

Gillon, M., Demory, B. O., Benneke, B., et al. 2012, A\&A, 539, A28

Giménez, A. 2006a, A\&A, 450, 1231

Giménez, A. 2006b, ApJ, 650, 408

Gladman, B. 1993, Icar, 106, 247

Goździewski, K., Bois, E., Maciejewski, A. J., \& Kiseleva-Eggleton, L. 2001, A\&A, 378, 569

Green, G. M., Schlafly, E. F., Finkbeiner, D. P., et al. 2015, ApJ, 810, 25

Grimm, S. L., Demory, B.-O., Gillon, M., et al. 2018, A\&A, 613, A68

Hadden, S., \& Lithwick, Y. 2016, ApJ, 828, 44

Hadden, S., \& Lithwick, Y. 2017, AJ, 154, 5

Haghighipour, N., \& Kaltenegger, L. 2013, ApJ, 777, 166

Hairer, E., Lubich, C., \& Wanner, G. 2006, Geometric Numerical Integration. Structure-Preserving Algorithms for Ordinary Differential Equations, Springer Series in Computational Mathematics (2nd ed; Berlin: Springer)

Hartman, J. D., Bakos, G. Á., Kipping, D. M., et al. 2011, ApJ, 728, 138

Hauschildt, P. H., Allard, F., \& Baron, E. 1999, ApJ, 512, 377

Haywood, R. D., Cameron, A. C., Queloz, D., Barros, S. C. C., \& Deleuil, M. 2014, MNRAS, 443, 2517

Henden, A. A., Levine, S., Terrell, D., \& Welch, D. L. 2015, AAS Meeting Abstracts, 225, 336.16

Hinse, T. C., Haghighipour, N., Kostov, V. B., \& Goździewski, K. 2015, ApJ, 799,88

Holczer, T., Shporer, A., Mazeh, T., et al. 2015, ApJ, 807, 170

Holman, M. J., \& Wiegert, P. A. 1999, AJ, 117, 621

Howard, A. W., Sanchis-Ojeda, R., Marcy, G. W., et al. 2013, Natur, 503, 381

Jontof-Hutter, D., Ford, E. B., Rowe, J. F., et al. 2016, ApJ, 820, 39

Jontof-Hutter, D., Lissauer, J. J., Rowe, J. F., \& Fabrycky, D. C. 2014, ApJ, 785,15

Jontof-Hutter, D., Rowe, J. F., Lissauer, J. J., Fabrycky, D. C., \& Ford, E. B. 2015, Natur, 522, 321

Kipping, D. M. 2013, MNRAS, 435, 2152

Kipping, D. M., Nesvorný, D., Buchhave, L. A., et al. 2014, ApJ, 784, 28

Kirk, B., Conroy, L., Prša, A., et al. 2016, AJ, 151, 68

Kley, W., \& Haghighipour, N. 2014, A\&A, 564, A72
Kley, W., \& Haghighipour, N. 2015, A\&A, 581, A20

Koch, D. G., Borucki, W. J., Basri, G., et al. 2010, ApJL, 713, L79

Kopparapu, R. K., Ramirez, R., Kasting, J. F., et al. 2013, ApJ, 765, 131

Kopparapu, R. K., Ramirez, R. M., \& SchottelKotte, J. 2014, ApJL, 787, L29

Kostov, V. B., McCollough, P. R., Carter, J. A., et al. 2014, ApJ, 784, 14

Kostov, V. B., McCullough, P. R., Hinse, T. C., et al. 2013, ApJ, 770, 52

Kostov, V. B., Orosz, J. A., Welsh, W. F., et al. 2016, ApJ, 827, 86

Kratter, K. M., \& Shannon, A. 2014, MNRAS, 437, 3727

Lee, J. W., Hinse, T. C., Youn, J.-H., \& Han, W. 2014, MNRAS, 445, 2331

Lines, S., Leinhardt, Z. M., Baruteau, C., Paardekooper, S.-J., \& Carter, P. J. 2015a, A\&A, 582, A5

Lines, S., Leinhardt, Z. M., Baruteau, C., Paardekooper, S.-J., \& Carter, P. J. 2016, A\&A, 590, A62

Lines, S., Leinhardt, Z. M., Paardekooper, S-J., Baruteau, C., \& Thebault, P. 2015b, ApJL, 782, L11

Lissauer, J. J., Jontof-Hutter, D., Rowe, J. F., et al. 2013, ApJ, 770, 131

Lissauer, J. J., Marcy, G. W., Rowe, J. F., et al. 2012, ApJ, 750, 112

Lopez, E. D., \& Fortney, J. J. 2014, ApJ, 792, 1

MacDonald, M. G., Ragozzine, D., Fabrycky, D. C., et al. 2016, AJ, 152, 105

Mandel, K., \& Agol, E. 2002, ApJL, 580, L171

Marcy, G. W., Isaacson, H., Howard, A. W., et al. 2014, ApJS, 210, 20

Mardling, R. A., \& Lin, D. N. C. 2002, ApJ, 573, 829

Marsh, T. R. 2018, in Handbook of Exoplanets, ed. H. J. Deeg \& J. A. Belmonte (Cham: Springer), 96

Martin, R. G., Armitage, P. J., \& Alexander, R. D. 2013, ApJ, 773, 74

Marzari, F., Thebault, P., Scholl, H., Picogna, G., \& Baruteau, C. 2013, A\&A, 553, A71

Masuda, K. 2014, ApJ, 783, 53

Mazeh, T., Holczer, T., \& Shporer, A. 2015, ApJ, 800, 42

Meschiari, S. 2012, ApJ, 752, 71

Mills, S. M., \& Fabrycky, D. C. 2017, ApJL, 838, L11

Mills, S. M., Fabrycky, D. C., Migaszewski, C., et al. 2016, Natur, 533, 509

Motalebi, F., Udry, S., Gillon, M., et al. 2015, A\&A, 584, A72

Murray, C. D., \& Dermott, S. F. 1999, Solar System Dynamics (Cambridge: Cambridge Univ. Press)

Narita, N., Hirano, T., Fukui, A., et al. 2015, ApJ, 815, 47

Nelson, B., Ford, E. B., \& Payne, M. J. 2014, ApJS, 210, 11

Ofir, A., Dreizler, S., Zechmeister, M., \& Husser, T. O. 2014, A\&A, 561, A103

Orosz, J. A., \& Hauschildt, P. H. 2000, A\&A, 364, 265

Orosz, J. A., Welsh, W. F., Carter, J. A., et al. 2012a, ApJ, 758, 87

Orosz, J. A., Welsh, W. F., Carter, J. A., et al. 2012b, Sci, 337, 1511

Osborn, H. P., Santerne, A., Barros, S. C. C., et al. 2017, A\&A, 604, A19

Paardekooper, S.-J., Leinhardt, Z. M., Thébault, P., \& Baruteau, C. 2012, ApJL, 754, L16

Pelupessy, F. I., \& Portegies Zwart, S. 2013, MNRAS, 429, 895

Pepe, F., Cameron, A. C., Latham, D. W., et al. 2013, Natur, 503, 377

Pierens, A., \& Nelson, R. P. 2008, A\&A, 483, 633

Pierens, A., \& Nelson, R. P. 2013, a\&A, 556, A134

Prieto-Arranz, J., Palle, E., Gandolfi, D., et al. 2018, A\&A, 618, A116

Prša, A., Batalha, N. M., Slawson, R. W., et al. 2011, AJ, 141, 83

Qian, S.-B., Liu, L., Zhu, L.-Y., et al. 2012a, MNRAS, 422, L24

Qian, S.-B., Shi, G., Zola, S., et al. 2013, MNRAS, 436, 1408

Qian, S.-B., Zhu, L.-Y., Dai, Z.-B., et al. 2012b, ApJL, 745, L23

Quarles, B., \& Lissauer, J. J. 2018, AJ, 155, 130

Quarles, B., Satyal, S., Kostov, V., Kaib, N., \& Haghighipour, N. 2018, ApJ, 856,150

Rafikov, R. R. 2013, ApJL, 764, L16

Raghavan, D., McAlister, H. A., Henry, T. J., et al. 2010, ApJS, 190,

Rein, H., \& Liu, S. 2012, A\&A, 537, A128

Sanchis-Ojeda, R., Fabrycky, D. C., Winn, J. N., et al. 2012, Natur, 487, 449

Santerne, A., Brugger, B., Armstrong, D. J., et al. 2018, NatAs, 2, 393

Schmitt, J. R., Agol, E., Deck, K. M., et al. 2014, ApJ, 795, 167

Schneider, J. 1994, P\&SS, 42, 539

Schwamb, M. E., Orosz, J. A., Carter, J. A., et al. 2013, ApJ, 768, 127

Schwarz, R., Haghighipour, N., Eggl, S., Pilat-Lohinger, E., \& Funk, B. 2011, MNRAS, 414, 2763

Silva Aguirre, V., Davies, G. R., Basu, S., et al. 2015, MNRAS, 452, 2127

Sinukoff, E., Howard, A. W., Petigura, E. A., et al. 2016, ApJ, 827, 785

Sinukoff, E., Howard, A. W., Petigura, E. A., et al. 2017, AJ, 153, 271

Slawson, R. W., Prša, A., \& Welsh, W. F. 2011, AJ, 142, 160 
Smith, A. W., \& Lissauer, J. J. 2009, Icar, 201, 381

Southworth, J. 2011, MNRAS, 417, 2166

Southworth, J., Mancini, L., Madhusudhan, N., et al. 2017, AJ, 153, 191

Tegmark, M., Strauss, M. A., Blanton, M. R., et al. 2004, PhRvD, 69, 103501

Ter Braak, C. J. F. 2006, S\&C, 16, 239

van Grootel, V., Gillon, M., Valencia, D., et al. 2014, ApJ, 786, 2

Vanderburg, A., Montet, B. T., Johnson, J. A., et al. 2015, ApJ, 800, 59 von Braun, K., Boyajian, T. S., Kane, S. R., et al. 2012, ApJ, 753, 171 Weiss, L. M., Marcy, G. W., Rowe, J. F., et al. 2013, ApJ, 768, 14 Weiss, L. M., Rogers, L. A., Isaacson, H. T., et al. 2016, ApJ, 819, 83 Welsh, W. F., Orosz, J. A., Carter, J. A., et al. 2012, Natur, 481, 475 Welsh, W. F., Orosz, J. A., Short, D. R., et al. 2015, ApJ, 809, 26

Winn, J. N., Matthews, J. M., Dawson, R. I., et al. 2011, ApJL, 737, L18 Wisdom, J., \& Holman, M. J. 1991, AJ, 102, 1528 